\title{
A New Procedure for Weighted Random Built-In Self-Test
}

\author{
Fidel Muradali \\ Bachelor of Engineering \\ Department of Electrical Engineering \\ McGill University
}

A thesis submitted to the Faculty of Graduate Studies and Research in partial fulfillment of the requircments for the degree of Master of Engineering

(C) Fidel Muradali March 1990. 


\section{Table of Contents}

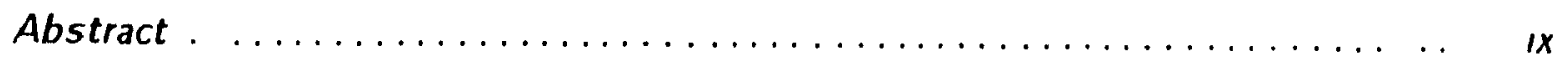

Résumé . . . . ..................... .

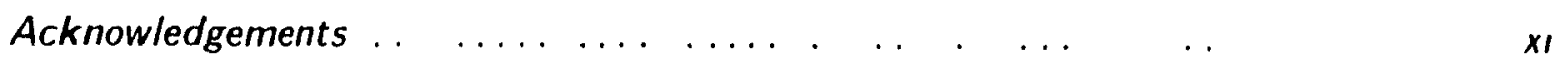

Chapter 1 Introduction... ............... 1

Chapter 2 An Overview of Testing \& Test Set Generation . 4

2.1 Failures and Fault Models $\ldots \ldots \ldots \ldots \ldots \ldots \ldots \ldots \ldots$

2.2 Approaches To Testing - Functional \& Structural Tests ... . . . . . . 6

2.3 Test Pattern Generation... $\ldots \ldots \ldots \ldots \ldots \ldots \ldots \ldots$

2.3.1 Algorithmic Test Pattern Generation (ATPG) $\ldots \ldots \ldots$

2.3.2 Random Pattern Based Test Pattern Generation . 8

2.3.2.1 Simulation Based Random Test Pattern Generation (RTPG) . . . . . . . . . 10

2322 Non-Simulation Based Random Pattern Test . . 10

$\begin{array}{lll}\text { Chapter } 3 \text { Design for Testability . } & 12\end{array}$

3.1 Ad-Hoc Approaches $\ldots \ldots \ldots \ldots \ldots \ldots \ldots \ldots \ldots \ldots \ldots \ldots \ldots \ldots$

3.1.1 Partitioning ... . . . . . . . . . 13

3.1.2 Test Point Insertion.................. . 13

3.2 Structured Approaches . . . . . . . . . . . . . . . . . $\quad 13$

3.2.1 Full Scan Design $\ldots \ldots \ldots \ldots \ldots \ldots \ldots \ldots \ldots \ldots \ldots \ldots \ldots$

3.2.2 Boundary Scan $\ldots \ldots \ldots \ldots \ldots \ldots \ldots \ldots, \ldots \ldots, \ldots, \ldots, \ldots, \ldots$

Chapter 4 Practical Issues in Testing ... . . 17

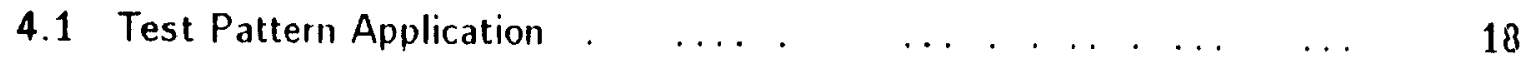

411 Stored Pattern Testing . . . . ....... . $\quad 18$

4.1.2 Hardware Pattern Generation . . . . . . . . . . . . . . . . 19 
4.13 Linear Feedback Shıft Registers 21)

414 Cellular Automata 22

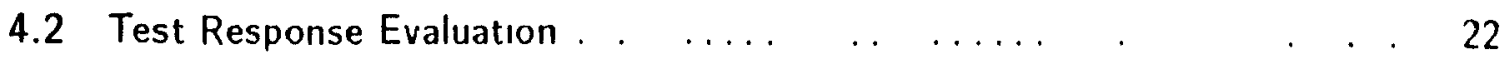

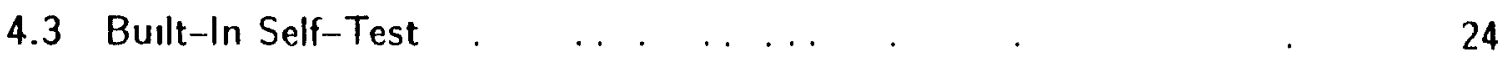

431 Some BIST Test Pattern Generation Methods 26

Chapter 5 Testing With Weighted Random Patterns 28

5.1 Some Algorithms to Determıne Weight Sets . . . . . 28

5.2 Weighted Generators . . . . 38

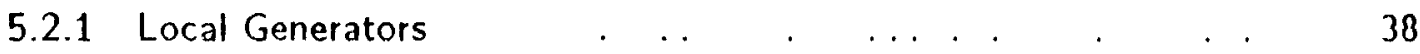

52.2 Global Weighted Pattern Generators $\ldots \ldots \ldots \ldots \ldots \ldots$. . . . .

Chapter 6 Weighted Random Built-In Self-Test 45

61 Weight Set Estimation ... . . . . . . . . . . . . 46

6.1.1 Processing the Tail Vectors (B) . . . . . 49

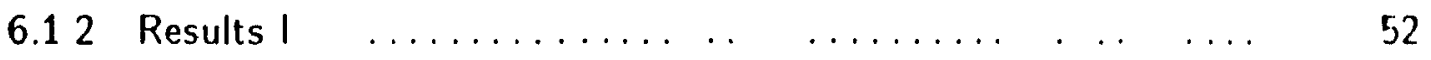

62 Improving the Estımated Weight Set (C.D) . . . . . . 54

6.2.1 Phase 1 - Update Target Pool (C) .......... . ... 54

6.21 .1 Results $\| \ldots \ldots \ldots \ldots \ldots$

6.2.2 Phase 2 - Relax Pin Biases (D) ....... . . . 55

62.21 Results III . $\quad \ldots \quad \ldots \quad \ldots$

6.3 Hardware Implementation . . . . . . . . . 57

6.31 Local Generation of Dually Weighted Test Patterns 58

63.2 The Circuit 59

6.3.3 Mixed Generation/Application of Uniform and Weighted Patterns ................................ 61

6.34 Area Penalty ............... . . 63

6.35 Quantization of Pin Biases (E). . . . . . . 64

6.3.5.1 Results IV .................... 64 
6.3.6 LFSR-Based Scheme $\ldots \ldots \ldots \ldots \ldots 6$

64 Other Test Circuits $\quad$. $\quad 70$

65 Comments on Overhead and Testability. . . . . . 73

6.5.1 Computation Overhead . . . . . . . . . . . . . . . . . 73

6.5.2 Accuracy of Extracted Data ....... . ...... 73

65.3 Testability of Modified Scan cells . . . . . . . . . 74

6.54 Possible Missıng Input State (F). . . . . $\quad 76$

66 CONCLUSIONS . . . . . . . . . . . . . . . . 78

Appendix A Extended Experimental Results . . . . . . $\quad \ldots \quad \ldots$

References.$\ldots \ldots \ldots \ldots \ldots \ldots \ldots \ldots \ldots \ldots \ldots \ldots \ldots \ldots$ 


\section{List of Figures}

2.1 Operation of a Test Vector

2.2 Progression of Coverage with Pseudorandom Patterns

31 Full-Scan Design Concept

41 LFSR1 - polynomial divider - Characteristic Poly $x^{4}+x^{3}+1$

4.2 LFSR2 - Characteristic Poly $x^{4}+x^{3}+1$

43 MISR $-x^{4}+x^{3}+1$

4.4 Standard BIST Scheme

4.5 BIST of Separate Circuit Blocks

51 Failure of $[E, 187]$ due to Fanout

5.2 Local Weighted Pattern Generation . .... . . . . . . 38

5.3 Weighted Generator of $[$ Sch75] $\quad \ldots \quad \ldots$.

5.4 Global Weighted Pattern Generation . 40

5.5 Weighted Instruction Generator. . . . . 41

5.6 Cascade AND Weight System . . . . . . . . $\quad 42$

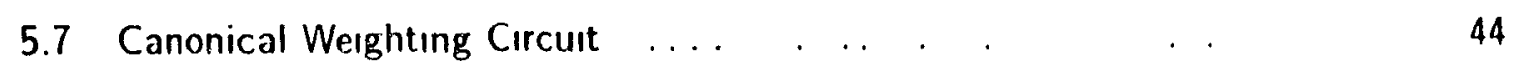

6.1 Flowchart of the Weight Estimation Procedure . . . . . . . . . . 17

62 Progression of Coverage with Uniform Random Patterns 48

6.3 Expected effect of 2 weight sets 49

6.5 Generic Scan cell... . . . . . . . . 59

6.4 Distributed Generation of Patterns according to 2 weight sets 59

66 Scan celi modified for WRP generation

67 GSCAN cell for a bias of $1 \quad \ldots \quad \ldots \quad \ldots \ldots \ldots$

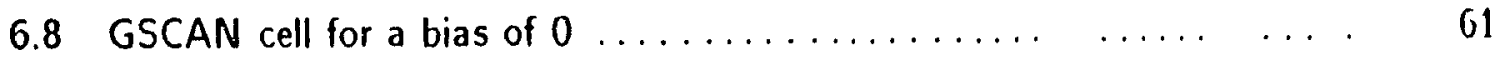


69 GSCAN cell for a bras of $025 \ldots \ldots$. . . . . 61

610 GSCAN cell for a bias of 075 . 62

6.11 Block Diagram of WT-SEL Generation . . . . . . . . . . . . 62

612 Thresholdıng conditıons for Quantization Runs ............. 65

613 Coverage Plots for C6941 $\quad \ldots . . \quad 68$

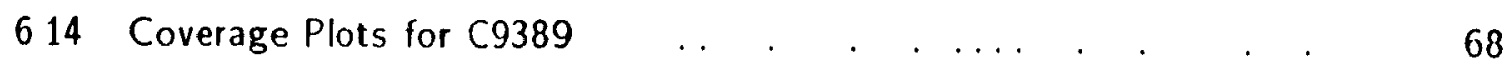

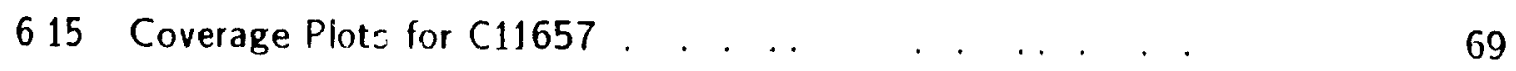

6.16 Coverage Plots for C30989 . . . . . . . . . 69

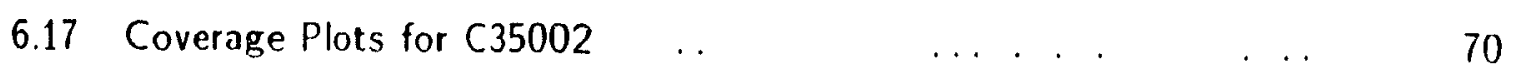

6.18 Sample Circuit for Bit Flippıng . . . . . . . .

619 Introduced Fault Site; for a Modified Scan Cell .

620 Scan Chain Ordering for Possible Missing State 76

A.1 Coverage vs Application Time Plots for C6941 ... . . . . . . . 86

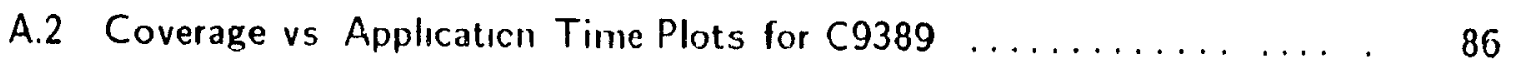

A.3 Coverage vs. Application Time Plots for C11657 . _ . . $\quad 87$

A.4 Coverage vs. Application Time Plots for C30989 . . . . . . . . . 87

A.5 Coverage vs Application Time Plots for $\mathrm{C} 35002$. $\quad . \quad . \quad . \quad 88$ 


\section{List of Tables}

5.1 Comparison of [Wun88] Data to Random Pattern Simulation Results

5.2 Backtrace Signal Probability Update Formulae [Bar87]

5.3 Weight Number(W1.1F0) Update Calculation [Eıc87)

61 Reducing the contribution of multiple detection of faults

6.2 Weight Set Estımation

63 General Circuit Ififormation

64 Initial (random pattern) Circuit Data

6.5 Initial WRP data

6.6 Phase1 runs of $40 \mathrm{~K}$ length

67 Refined WRP Data

68 Weighted Testıng $\vee s$ Random Testıng

6.9 Modes of operation for WT-SEL

6.10 Quantization Runs for Case 1 - 7 bias levels .. .

6.11 Quantization Runs for CASE 4 - 5 bias levels

6.12 Comparison of IFSR-based Mixed-Weighted Scheme and Random Pattern Testing

6.13 General Circuit Information

6.14 Initial (random pattern) Circuit Data

6.15 Extended Uniform Random Pattern Circuit Data . . . . . . .

6.16 Weighted Testing v s Random Testing

6.17 Bit flippıng for sample circuit

6.18 patterns 


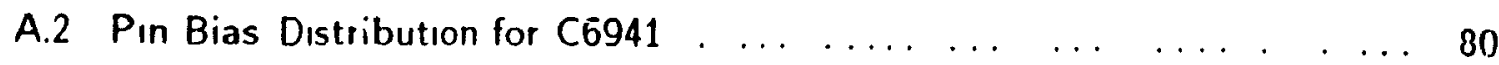

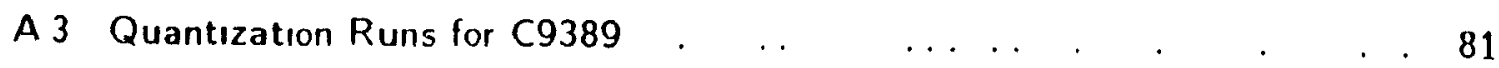

A.4 Pin Bias Distribution for C9389 f $\quad \ldots \ldots \ldots$

A 5 Quantization Runs for C11757 . 82

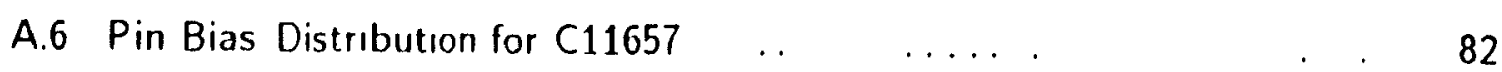

A 7 Quantization Runs for C30989 . . . . . . . . . . . . . . . . 83

A 8 Pin Bias Distribution for C30989 . . $\quad$. 83

A.9 Quantization Runs for $\mathrm{C} 35002 \ldots \ldots \ldots$

A 10 Pin Bias Distribution for C35002 . . . . . . . ... . . . . . 84

A 11 Extended Results for the modeled BIST Implementation with 32 bit LFSR 
Experience has shown that an excessive time penalty can be mcurred when test ming lank" scan circuits with a uniform random test pattern generation approach 4 s a solution 1 ." this problem, this work explores the use of weighted andom pattens (WRP) toredure. by orders of inagnitude, the test application time in self-lesting ciocuits

Much work has been done on the off-line development of compact test set s. but a problemul which still remains is how to efficiently apply them on-chip. A means of transforming a given test set into a relatively short weighted sequence and pseudorandonil sefunemen. whose cumulative fault coverages approximate that guaranteed by the miginal lest set. is proposed.

The single weight set is formulated using a method which does not explictilv consirlen the circuit structure. Instead, sufficient circuit unformation contained in the given lent set can be extracted using smmulation techniques This is done br analvzmng a ramlon! pattern detection profile and isolating the vectors which cover faults diflic ult Io deren 1 using random patterns. After extracing the useful tits frorn these vectors, a weight set characteristic of the corresponding faults is estimated as the ratio of l's to 0 's at cach bit (Ir.put) position.

The generation scheme is evaluated using five large scannable circuits. $\Lambda$ local approach to on-chip pattern generation is examined. 


\section{Résumé}

L'expérimentation démontre que la vérification de circuits complexes aver chaille do balavage par une genération aléatoire uriforme de palrons de test peill s'avérer trècs longue Carte recherche étadie l'utilisation de patrons aléatoires pondérés pour diminner significativement le temps de test des circuits auto-vérifiants.

P'lusieurs recherches sur le développement d'ensemble compact de patrons de test onl été effectuées mais leur utilisation efficace à l'intéricur mêne des puces demeure in problème. Nous propozons une façon de transformer un ensemble donné de patrons de test en une relativement courte séquence pondérée et une séquence pseudo-alćatoire. dont la couverure cumulative approxime celle de la séquence originale liensemble de poids est calculé selon une méthode qui ne consıdère pas explıcitement la structure du circuit Il est assumé que suffisament d'information est contenue dans l'eisemble des patrons de test

Les vecteurs qui couvrent des df́auts difficiles à détecter par des pations aléatrires sont. isolés en analysant un profile de patron aléatoire de détection. Les bits utiles à la détection sont isolés de chacun des vecteurs de test Un poids correspondant au rapport. du nombre de $\mathrm{i}$ sur le nombre de 0 est attribué à chacune des entrées. Un poids uniforme de .5 est utilisé pour couvrir les autres défauts vérifiables.

Le procédé do génération a été evalué en utilisant cinq gros circuits à chaine de balayage. Une approche locale est présentée pour la génération interne de patrons de test. 


\section{Acknowledgements}

I would like to take this opportunity to thank my supervisor Dr. I. K. Agarwal for liki guidance throughout my term as a graduate student, and Benoit. Nadenu-Dostic for his invaluable insight and pracrical suggestions which led to a more realistic ar,lution to the problems addressed in this thesis. Also, to Rob Aitken for taking on the undesilahlo" task of proof reading the text, and to Francis Larochelle for his french translition skills. I would especially like to thank Ashısh Panchoiy for assisting with the postreview corrections in my absence Finally, a "thanks guys/gals" goes ont to all the liah, members for making studying an enjoyable experience.

This work was supported in part by a grant froin Fonds pour la Formation de ('hercheurs et, L'Aide à la Recherche (FCAR) and test circuits were provicled by Bell Northern Research (BNR). 
Today it is not uncommon to find VLSI chips containing hundreds of thousands to over a million circuit elements, and with continued refinements to packaging and submicron fabrication technologies, circuit density is expected to increase. Inevitably the question must be asked, "...but does it work?" The field of testing endevours to respond to this concern.

In modern circuits, testing accounts for roughly a third of a chip's production cost [Bha89]. In fact, it has been found [Wil83] [Bar87] that the price of testing increases approximately five to ten times per level of packaging, to the point where thousands of dollars are at stake if tests are performed in the field. Thus, one way to reduce long term test costs is to ensure that component tests are as thorough as possible at earlier stages of assembly (e.g. probe and chip levels). Also, if possible, the design of the circuit itself should facilitate decreased test effort and increased test effectiveness. Such linking of the design process with testing has resulted in a knowledge hase of design techniques called "Design for Testability" (DFT). An example of this is built-in selftest (BIST) wherein on-chip and/or on-board circuits provide and analvze test, data. The technique substantially reduces the dependency on external test unit,s and can thus simplify in-field maintenance.

This thesis demonstrates the use of DFT concepts to develop a new BIST strategy intended for large circuits (hundreds of thousands of gates) with thousands of $1 / 0$ signals. In general, the amount of input data needed to test a circuit is proportional 
to the square of the number of circuit gates [Goe81] As such the storage menuirements of the test hardware and test application time also grow q'tadratically with circuit size Thus as circuit density approaches that of the curcuits under constleration, these factors may become bottlenecks which affect the cost ol performing a through test

In test generation method proposed, input data is psendorandomly generaled in-r irrull to decrease the storage requirements needed. Furthermore, in addition to using a conventional uniform random pattern generation approach |Bar87|, the volume of inpult. data is significantly reduced by generating non-uniform, or weighted, Idmlom palle's"l sequences. Experimental results for this scheme record test times orders of magnitude smaller than that of standard uniform random pattern testing

The structure of this thesis follows the breakdown of the test process into two off-line steps :

- Test generation, and

- Test set verification

and 2 practical steps :

- Test pattern application, and

- Test response evaluation

However, the emphasis is placed mainly on the front-end test generation and test application processes.

Chapter 2 introduces the concepts involved in test generation. This is basic ally $1 /$. compilation of a set of input stimuli which verifies whether a circust is defer tive or mint Test set verification is the evaluation of the effectiveness of a given tost set to this mol This is usually recorded as a measure of the percentage of morleled laults whirh an be detected This measure is called the fault coverage of the lant set Acorrling In the manner in which the test set is developed verification mav be rone implicitly (e $\mathrm{g}$ ATPG section 2.3.1), or with the use of simulation which is a brute force approach Further discussion of test set verification is not micluded in this thesis.

As a transition from the off-line steps to the practical issues, DFT techniques pertincut. 
to the research at hand are summarized in chapter 3 . Chapter 4 then rescribes somm of the hardware used to physically apply test input to the circuit under lest (CI'I). and analyse the CUT's response to this data. An introduction to on-chip testing is also given in this chapter.

Chapter 5 is a brief overview of some known algorithms and circuits used to generate weighted random patterns. Chapter 6 contains a detailed examination of the proposed weighted random BIST strategy, and comments on the computational overhearl repuired and the testability of the circuits designed. Supplementary details concerning the experiments performed are contained in the appendices. 
A digital circuit responds to discrete potentials asserted at its input lines, alll, as a result, asserts another set of these "digital signals" at its output lines. In general, thr value of a digital signal is restricted (within a threshold) to logical 1, corresponding to power supply potential, and to logical 0, corresponding to ground $\Lambda$ single enumeration of innut signals applied to a circuit is called an "input vertor" or "input pattern" to that circuit, and likewise, the corresponding collection of outpul values is an "outpul vector" or "output pattern". A combinatıonal circuit is one which does not contain memory, thus its output state depends only on the input vector applierl. On the other hand, due to the presence or memory, successive states of a sequentral circ uit ale reldien This implies that a specific sequence of input vectors may be required in order to forc" a sequential circuit into a particular output state.

This chapter introduces some of the ideas involved in automatically generating vertors for testing combinational circuits. The same task, if performed with resper t to serfuential circuits is considerably more computationally intensive [Set85] Fortunately though, through the use of existing "scan design" techmiques (covered in chapter:3). ce(fuential structures can be temporarily converted into combinational ones for testung purperm Thus, sequential circuits in a scan-based design environment are also included in thr discussion. 


\subsection{Failures and Fault Models}

Testing is the process in which a circuit's function or structure is validated This is done by attempting to force a circuit into a known state and comparing the observerl result. with the expected response. A mismatch of these values implies that the circ nit conlains a "failure" and is imperfect Failures are physical anomalies within a fabricated circuit which cause it to malfunction Notwithstanding design errors, they may be the esull of imperfections in the fabrication pocess leading to device flaws inc luding shorts heotwern conductors, broken interconnects, improperly doped regions and missing contacts Thev may also occur as in--,ervice deferts caused by, for instance, metal migration or power overload. Failures may manifest themselves as parametric errors or affect the steadvstate of the circuit.

In order to generate tests, a "fault model" should be devised to be representative of many, if not all, fallures which can occur. To this date, a single model which can characterize all possible defects has not been formulated. The industry standard for combinational testing is the "single stuck-at" model |Eld56| which assumes that under the influence of a fault, a line is expected to be held at a fixed logic value irrespective of the polarity of the driving signal. Thus the line is said to be 'stuck' at a logical 1 or 0 . For example, a NAND gate with a stuck-at 1 (s-a-1) fault on its output will assert an output value of 1 regardless of its inputs.

A second stipulation of the single stuck-at model is that a faulty circuit is assumed to contain only 1 fault If multiple faults were considered, in an $n$ line circuit there could be up to $3^{n}-1$ possible faulty circuit representations since a line can be s-a-1, s-a-0 or fault-free. When a single stuck fault is assumed, the number of possible faulty cases is reduced to $2 n$.

Although the single stuck-at fault assumption has been sufficient in practice |Wil83|. there are some CMOS defects which cannot be properly handled using this model. In particular, some faults introduce memory effects into the circuit, thus a specific ordering of test vectors may be required for detection. An example of this is stuck-open faults 
22 Approaches To Testing - Functional \& Structural Teats

[Wad78] and a class of bridging faults [Mei74] which create nnwanted lomilhark pallis

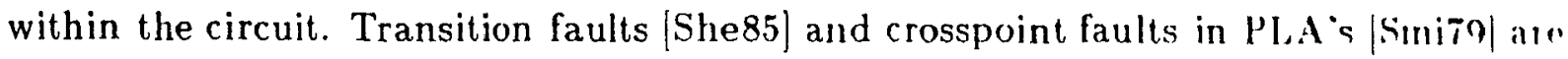
other faults which the stuck-at model does not explicitly take into account

Nevertheless, because of its computational simplicity and since practical experience hats shown that a complete stuck-at test also tends to detect a large number of other lault types |Wil83| (termed "windfall" fault coverage), the fault model used for this rescarch is the single stuck-at model.

\subsection{Approaches To Testing - Functional \& Structural Tests}

There are two approaches to generating test vectors These are hased on wherlier il functional or structural iepresentation of the circuit is processed $\mid$ Gid893| Mank9|

Functional testing usually requires in-depth knowledge of the circuit's operation but tends to neglect explicit hardware detals. This approach herarchically cheeks the circuit and internal circuit modules by verifying that their intended functionality and interaction as a unt is according to specification for example, functional testing letermines if adders combine bits properly, if memories can be accessed and if ALI's 'mrform all desired operations Functional tests are commonly developed for design verification

Structural testing attempts to give a level of assurance that the (UTT will oprrate correctly by ensuring that its components (gates, lines, etc.) do not rontain faultis Development of these tests requires knowledge of the operation of only the basic rirc nit elements (e.g. logic gates and blocks such as flip flops and adders), and thus can be automated.

\subsection{Test Pattern Generation}

Testing a particular circuit node involves a path sensitization process in lliat all injul test vector is generated which: 
- stimulates the CUT inputs to force or control the desired node into thr known fault-free state, and

- stimulates the CUT inputs so that the effects of this assignment ran bo propagated and observed at the circuit outputs

Figure 21 illustrates how parts of a test vector controls and observes a fault site In 2.1i. a s-a-0 on the output of gate 2 is to be tested. The input assignment $11 \times x$ controls the target, line to a 1 (fault free value) Next, in order to ohserve llie effects of the potential fault, a path must be sensitized from the faulted line to the circuit oulpul Thus, the output of gate 3 must be a non-controlling value with respect to the output AND (gate 4) This is done in figure $2.1 \mathrm{~b}$ by the input vector $x \times(00$ Since there is no conflict between the vectors required to control and observe the fault, the final test vector is 1100 .

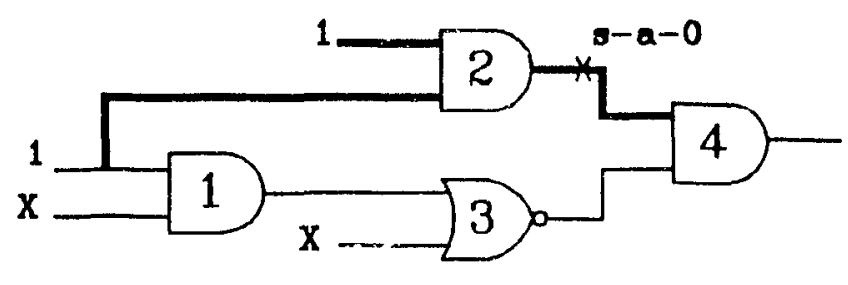

(i) - control

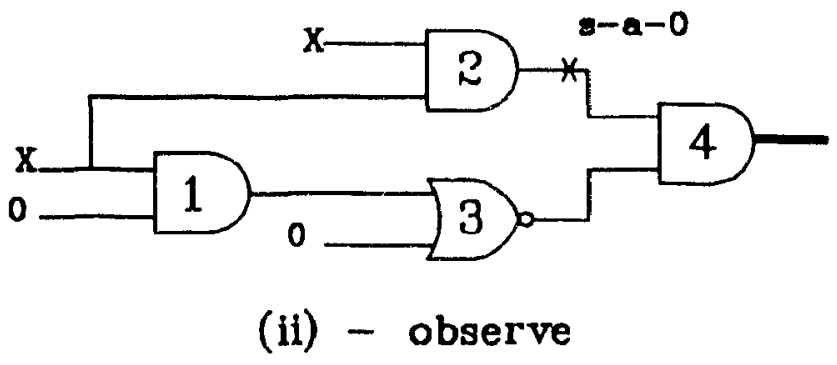

Figure 2.1 Operation of a Test Vector

Cenerating a test vector for a given fault belongs to a class of problems known as "NP complete". This means that the complexity of the task may increase exponentially with the size of the input (in this case the number of circu,t lines) under consideration |Nil8(0| 
As a result, heuristics (see references of section 2.3 .1 ) are designed which attempt to reduce the amount on computational overhead encountered in dev eloping test sets.

There are 2 approaches to generating a deterministic test set:

- Algorithmic test pattern generation

- Random pattern based test pattern generation

\subsubsection{Algorithmic Test Pattern Generation (ATPG)}

For each potentially detectable fault in a circuit, ATPG tools altempt to analvti. cally design a test vector by implementing a path sensitization process This inlon has existed for roughly 2 decades now and originaterl with the I)-algorithm | Rotrifi| Some improved methods developed through the years include fielfig|(boolean difference), [Goe81](PODEM), |Fuj83](FAN), |Ake76], [Sch88|(SOCRATESi) and most rocently $[$ Cox90](C:AMP)

\subsubsection{Random Pattern Based Test Pattern Generation}

The premise here is that a sufficiently large number of pseudorandomly generated lent vectors can detect most, if not all, faults. The typical result of this trial and error approach is shown graphically in figure 2.2 .

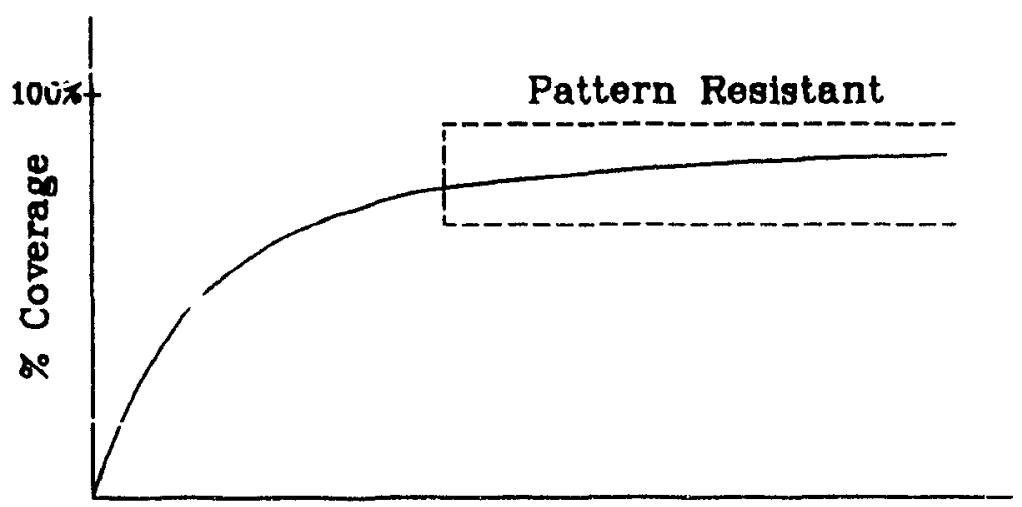

No. of Patterns

Figure 2.2 Progression of Coverage witlı Pselıdorandom Patterns 
It is common that a large portion of the detectable faults are covered within a relatively small number of input vertors, resulting in a large positive slope in the progression of coverage graph Ilowever, there exists a group of laults, classified as "pattern lesistanl" which, if retectable, may require test lengths orders of magnitude larger than the size of a deterministic test set The flattened tail region of the coverage graph correspond to the reldtive test lengths after which pattern resistant faults de deter ted.

There are a few reasons why a particular fault might be pattern resistant |Tot 88 |.

- Redundancy - the fault is not detectable

- Reconvergent fanout - there is a possibility of canccllation of fault cffor tis

- High fan-in - introduced correlation tends to inhubit propagation

- Test vector quality - e.g the generated distribution of 1 and 0 assignments on the CUT inputs is in conflict with what is required to control/observe many faults, or there exists correlation between surcessive patterns or hits and this correlation prohibits/mnedes fault detection

A potential remedy to the potentially unieasonable time penalty which may be incurred in detecting pattern resistant faults is a joint generation scheme - pseudorandom patterns are generated to detect a large group of faults after which ATPC is used to detect the rest $\left|K a w 88^{\circ}\right|$. As is demonstrated by this thesis, weighted random patterns can he also used to dramatically increase the rate of coverage compared to standard uniform random pattern generation.

One of the earliest applications of testing with a generated sequence is fault injection (also called fault insertion [Sus73]). This entails physically injecting a lault into a discrete model of the circuit built with 'off the shelf' components, followed by randomly' assigning a series of test stimuli. The test vectors which produce a response different than that of the known fault free circuit are retaned as tests for the injerted laults This classical method is suitable for small circuits (TTL. DTL) using low levels of integration but clearly such an approach is impractical with today's rircuit densities. The method is mimicked through the use of computer simulation.

1 in the course of this text 'random' or 'uniformly distributed random' generation refers to psendorandom generation in which there is an equal chance of a bit being assigned 1 or 0 


\subsubsection{Simulation Based Random Test Pattern Generation (RTPG)}

Similar to ATPG, a structural description of the curcuit structure is instructed in software, and appropriate modeled faults are associated with each circuit norle fin each pseudorandom vector applied, the simulator reproduces the result of the faullol and fault free circuit, and checks if any of the modeled faults are detected If ao, Ihr detected fault may be removed from the original fault set, and the pattern is petamml as a test vertor.

Such simulation based approaches can be used to provide compact delemmmislic lest sets. This is done in the following manner: The test vectors found nearing the chrl of the process detect very specific (more pattern resistant) faults and mav also cover many of those 'easily detectable' faults found earlier in the procedure. By simulating the extracted test set in reverse order to which the vectors were found and pruning the fault set after each vector, the size of the lest set can be reduced by about, $255^{10}$ to 50$)^{10}$ This procedure is commonly called reverse compaction

The complexity of fault simulation tasks is believed to be $O\left(n^{2}\right)|\operatorname{|lar} 87|$, where $n$ is the number of circuit gates This is mostly due to the existence of reconvergence which creates correlation between gate inputs. Some existing simulators are clescriber in |Wai85] and $\mid$ Maa87).

\subsubsection{Non-Simulation Based Random Pattern Test}

In order to eliminate the computational overhead of fault simulation. a 'auffic ientlv lomk' test sequence is applied to the CUT. Unlike section 2.32.1, although the lest set is never formally designed, it is assumed that it is contaned within the generated font serfuener

Since fault simulation is not performed, the fault coverage of the tegt sepuener can only be approximated. Sometimes a statistical sampling method |Siet85] is used to estumat" this value Here, a randomly selected sample of circuit faults is simulater using the sequence to be evaluated. The resulting fault coverage of this subset is used as an 
approximation of the overall coverage.

An estimate of the required test length, $N$, can be found using the formula :

$$
P_{N}(X)=\prod_{f \in F}\left(1-\left(1-p_{f}(X)\right)^{N}\right)
$$

where, $P_{N}(X)$ is the given threshold probability that each single fault $f$ in the original fault set $F$ is detected within a test sequence of $N$ test vectors. $p_{f}(X)$ is the detection probability of the fault $f$ [Gol74] [Brg84] [Jai84]. This is a measure of the oddls of detecting the fault $f$, and is dependent on the relative distribution $(x)$ of $1 \mathrm{~s}$ to $(\mathrm{k}$ at each bit position in the input sequence used. It has been found $\mid$ Sav8. that onlt the faults whose detection probabilities are roughly within a factor of 2 of the lowest. detection probability withn the fault set affect the test length estumate.

At the begining of this chapter it was mentioned that sequential curcuits can be temporarily converted into combinational structures. This idea of altering the structure of the CUT to ease testing tasks is elaborated upon in the following chapter. 
It was seen in Chapter 2 that testing for a circuit fault depends on the ahility to cont rol and observe a fault site The test circuit used to demonstrate the irlea (figure 21 ) was a fairly simple and small structure If however, the block was embedded in a much larger cell with a smaller pin to gate ratio, the ability to affect. the fanlt site may hr impaired As a result, testability analysis programs |Gol74] |Big84| |IdiRal| have heen developed to provide approximate measures of the controllability and obuervabilite of

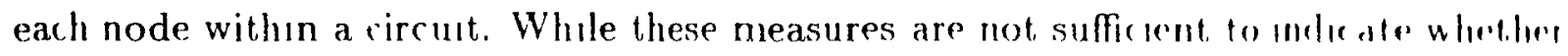
or not a specific fault is detected, the information can be used to locate a section of the CUT which is potentially difficult to test Agr82]. With this data. the circuit, an be modified to enhance its overall testability. Also. since theise algorithms are lom computation intensive than those designed to generate determmistic test vectors. the data is available relatıvely quickly during the design process.

The concept of altering the curcuit structure to simplify the test process essentially brings the test process directly into the design environment As such, an ever-g,owing knowledge base of design methods called, Design for Testability (DFT) /Wilk:3| k comst.ımlk being compiled. There are two DFT categories ad-hoc and structurenl. Examplem al each of these design approaches are given next.

\subsection{Ad-Hoc Approaches}

Ad-hoc DFT methods are usually device-specific and not intended to solve a general 
test problem. Two common strategies are logic partitioning and test point insertion.

\subsubsection{Partitioning}

Since the computation time for test pattern generation and evaluation is proportional les the number of gates squared for combinational circuits and cubed for sequential circuits |Set85|, a divide and conquer approach is taken to help reduce the test generation effort. Essentially the entire structure is subdivided into separate circuit blocks rluring the tost mode Depending on the interdependence between subcircuits, partitioned regions can be tested in parallel thus decreasing the overall test time

Modular or regularly design. l cells with natural partitions are more appiopriate for this strategy than are unstructured circuits designs. For instance, sorne designers exploit the natural inter-module isolation/communication of bus-based architectures to partition a circuit during test mode. However, with such an approach isolating bus fanlures tends to be a cumbersome process [How 89]

\subsubsection{Test Point Insertion}

As mentioned previously, regions of the CUT which are difficult to access can be identified. The controllability and observability of these sections can be physically enhancerl by inserking accessible test points which, during test mode, may act, as circuit inpuls, circuit outputs or both. A trivial example is to replace a circuit node by a flip-flop ronnerted in a scan-rhain (see sect 3.2 ). Selection of optimal insertion sites remains an open problem. Some results are discussed in [Lay 74][Hay 73][1ye89]

\subsection{Structured Approaches}

The intention of structured DFT is to introduce a design methodology to solve a general test problem. These technıques are usually geared for automated design.

Scan Design is probably the most popular structured DFT practice. It is based on providing access to circuit latches. There are several variations of the scheme, differing 
in latch, clocking and control designs imposed by in-house system de'sign rules |Wili's| [Ste77] [Eic78] [And80] [Nad88] [Fun89], but the underlying principle usuallv remains the same.

\subsubsection{Full Scan Design}

The intention of full scan designn methodology is to test a complex scquential circull by verifying that its structural build-up is sound ${ }^{2}$. In principle, for testing purposes. " sequential circuit is transformed into a combinational bloc $k$, thus cxisting combillation, test generation techniques can be employed. Such an approach is attractive because of the relative ease in generating tests for combinational as opposed to sequential rirc uits $\mid$ Set85|.

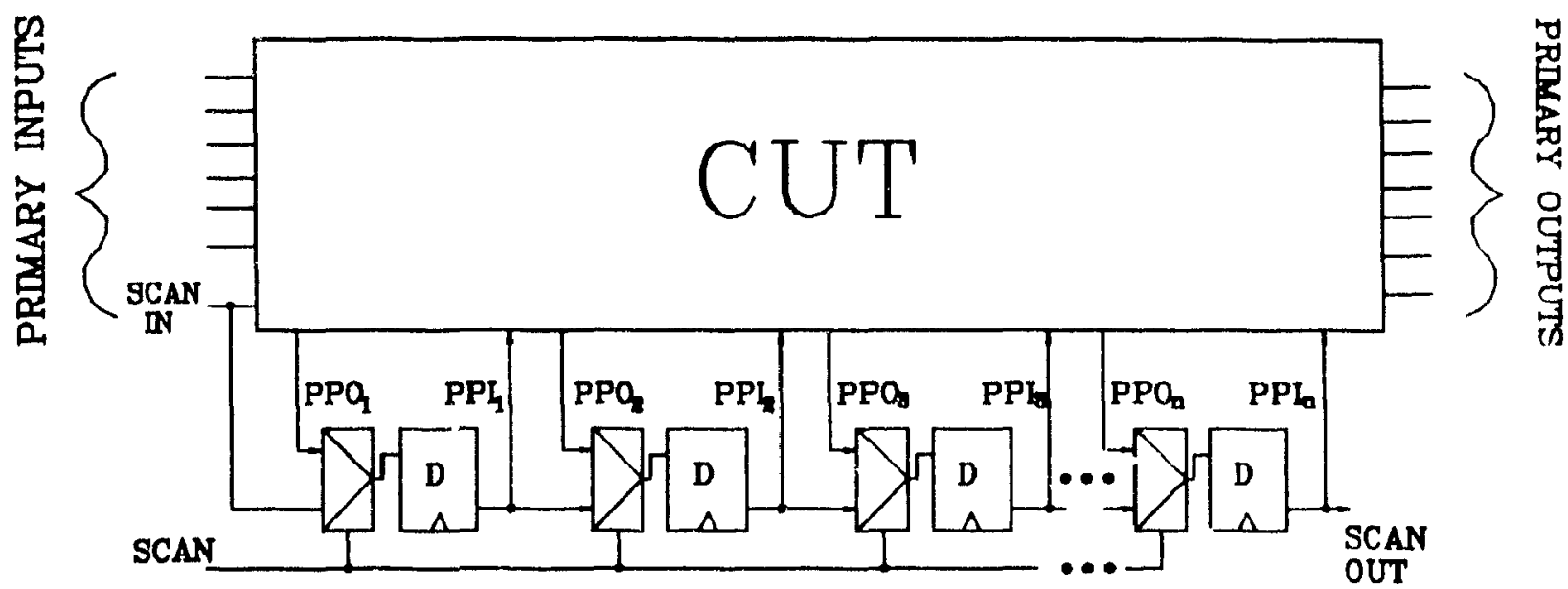

Figure 3.1 Full-Scan Design Concept

The basic full scan design concept is logically shown in figure $3.1^{3}$. Each system lateh. shown here as a $D$ type flip-flop (D-FF), is modified to accept multiplexed input one from the output of the previous scan cell. and the other from the circuit norle previously associated with the original latch input. An extra control line $(S C, \Lambda N)$ is needed to globally regulate the input selection of the multiplexers The result is the

2 A limited amount of functional vectors are also done for design verification and liming analysis

3 A good catalogue of techniques is contained in [McC85b] [Wil83] and [Fun89] 
creation of accessible pseudo-primary input nodes (PI' $I_{2}$ - previously the latch output.) and pseudo-primary output nodes $\left(\mathrm{PPO}_{2}\right.$ - previously the latch input), and the provision of at least two modes of operation - normal and scan.

As the narne implies, in normal mode the Hip-flops (and thus the svstem) performs with the intended functionality and the test circuitry is virtually transparent to the CUT. In scan mode, each scan rell accepts input from the output of a prederessor scan cell thus collectrvely joming to form a large shift register structure or scan-chain.

During the test procedure, for each test vector, the system is first placed into scan morle and, aside from the bits which map to primary inputs, the input vertor is seriallv shifted into the scan-chain. The CUT then returns to normal mode for 1 cvcle so that the circuit's response to this excitation can be losded into the scan-e hain Finally the system is again placed into scan mode and the test data contained in the chain is shifted out to be analysed. while another test vector is simultaneously shifted in.

The penalty for reducing the effort needed for test generation is an mrease in area, higher power consumption and possible operating specd degradation if test hardware is included in a critical path. Also, since test vectors are serially shifted into the chain, the time needed to apply the test is proportional to the size of the scan-cham userl. A partial solution is to introduce some parallelism by partitioning the scan-chain. Realistically, the number of these segments is limited by tradeoffs involving factors such as test time, area overhead and the pin limitations of both the external uster and (hup) |Bas89|

Some of the drawbacks of full scan design can be reduced by using a partial scan design |Pra88] [Che89| In this scheme, only a subset of the system latches are configureil inio a scan-chain. However, in such a system, the more difficult problems of sequential test generation and selection of optimal candidate sran cells must be consirlered

Despite the mherent disadvantages, many companies clamn that the polential simplification of the test pattern generation process justifies the use of full scan design |Wil8.3|. 


\subsubsection{Boundary Scan}

A board-level extension of chip-level full scan design is boundary scan |Cilo88| |Has88| [Par89|. In this technique the signals at the chip peripheries can be controlled and observed via scan cells associated with each of the primary input and output pins Boundary scan promises many advantages, such as enhanced board-level diagnosis, more standardized tests, and reduced test access problems, especially for in-circuit testing

As an alternative to the limitations imposed by the physical probes used in "berl-olnails" testing, boundary scan offers more reliable ssolation of circuit, nodes and climinalms the overdriving of internal lines. The gain is dramatic with high density single or dual sided surface mount boards $\mid$ Par89|.

Another structured DFT technique is to include test paltern generation and test. lesponse evaluation circuit to the chip or board under test. This is discussed in the next chapter. For further in. imation on DFT sce [Wil83]. 


\section{Chapter 4}

\section{Practical Issues in Testing}

As discussed in chapter 2 , much work has been conducted on off-line generation and verification of test sets and sequences. The second part of the test process, as defined in chapter 1 , is to devise a mechanism to physically apply the test, vectors to the CIT and analyse the response

The goal is to be able to detect all modeled faults. However, because of possible redundancies within the CUT, the total number of detectable faults may not be known (identifying redundancies is an NP-complete problem; unless a large amount of prepıocessing is done. So, the chorce of a testing strategy may be based on a tradeoff of many inter-related variables including .

- a pre-defined target level of faull coverage

- production quotas

- time needed to perfon the test

- on-chip area overhead introduced by DFT

- the effort required to implement DFT circuitry and perform the test,

- test equipment and circuit maintenance costs

Of course, no test scheme is universally adaptable because of the unpredictable advances in design technology and fault modeling However, current market orienterl dynamics should be accommodated. For example, with the current populanty of scan circuits, there will be a need for test strategies geared towards very dense circuits with thousands of serially accessed scan inputs/outputs [Bas89] Chapter 6 proposes a test generation/application procedure for such circuits. 
As a precursor to this, in the rest of this chapter, some common methods used for tint pattern application are outlined, followed by a short discussion of how the correspondint CUT response is analysed and evaluated. An introduction to on-r hup testing is also presented.

\subsection{Test Pattern Application}

Test quality and test application effort have a notable impact on the cost of testing inl integrated circuit. Most schemes can be categorized as:

- stored pattern testing, or

- hardware pattern generation

Ad-hoc store and generate and programmed schemes are sometimes adiled to the list. Iut. may be considered as extensions of the two approaches listed above.

\subsubsection{Stored Pattern Testing}

Stored pattern testing involves the application of specific deterministic test, vertors, mach of which provides an incremental level of coverage. Applying these vertors extermallv may require large storage capability and expensive test equipment, pricerl on the order of millions of dollars The technique is straightforward and suitable for many curmi testing needs. However, the specifications of a test unit are static . so upper limits an" physically imposed on such variables as test frequency. the number of available $1 /()$ channels, and the size of input, (pin) buffers On the other hand, devire rlaracteristire evolve in accordance with design and fabrication techolog arlancos, and usually mull in potentially higher operating speeds. higher pin counts and the need for progressivnlt larger test sets. This conflict ultimately implies costly test erquipment upgrarles "I replacement. It is questionable then, if long term testing is economically feasible will a standard stored pattern approach [Bas89]. 


\subsubsection{Hardware Pattern Generation}

In order to reduce the functional and memory requirements of the external tester required, relatively simple circuits, based on linear feedback shift registers (LFSRs) |Gol67| |Bar87| or cellular automata (CA) [Hor89], can be used to pseudorandomly generate tost sequences. In the most straightforward case, vectors are generated such that there is an equal probability of assigning a 1 or 0 to a CUT input. As mentioned in section 2.32 , it is intended that after a large number of these random vectors, a sufficiently high level of tault coverage can be achieved. Unfortunately, experience has shown (e.g. test lengths for C2670 and C7552 (ISC85|) that with current circuit densities, a number of these test. vectors orders of magnitude in excess of the maximum test length permited may be needed to attain this goal. Moreover a relatively small number of intial vectors cover a large portion of the detectable single stuck-at faults leaving the majority of the test sequence to be wasted in an attempt to detect random pattern resistant faults.

A possible alter native is to use a joint test strategy - apply a reasonable length of random test patterns and supplement this with stored pattern lesting. However, it has been found that in many cases, the size of the stored test sct, needed nears $70 \%$ of the full deterministic set [Bas89] Thus, this approach does not address the problem of limited storage.

Another possibility is the use of a store and generate approach (e g. |Agar81| |Abo8.3| [Bar85] (Fed86] [Eic87] (Ude88] [brg89]) where, relative to the size of a deterministic test set, a small number control words are used in conjunction with a randrom pat lern generator to produce a test sequence. Programmed testing is vet, another extension whereby functional test programs contained in a ROM generate the required test, set. This is common for microproressor based chips and systems | Bra84| |Kuh8.3|

Exhaustive testing uses a counter, LFSR or CA to apply a full functional test set (i.c. $2^{\prime \prime}$ distinct patterns where $n$ is the number of circuit inputs). This approach is guaranteed to detect all combinational fault, but since the test length increases exponentially with the number of CUT inputs, this type of test procedure may not be economical for 
circuits with with as few as 25 inputs. Thus partilioning can be emploved to pseude exhaustively [McC84] [Ude88] test the CUT as a set of smaller subcircuits. In sult approaches, care is must be taken to minimise the sizable amount of control needed for in-circuit partitioning.

There also exists a small group of regularly structured circuits, whose functionalih permits the ad-hoc design of very simple dedicated pattern generators Parity Im testing [Hon81] is an example. However, such solutions are, by far, the exception rather than the rule.

As will be shown in Chapter 5, generating test patterns in which there is a monl-11mform distribution of 1 's to 0 's can result in generated test lengths which are a small fiaction of those otherwise needed for uniform random pattern generation. One drawback of most existing such weighted generation hardware is that while much simpler than stored pattern testing, these generators are much more complicated than therr umlform random counterparts. However, chapter 6 describes a weighted genrratıon schemr which balances hardware complexity and test length at the expense of an accoptal,h. area penalty.

The root generator of many pattern generation schemes is a uniform random pallern generator often implemented using an LSFR or CA. The next two subsections introdur" these structures.

\subsubsection{Linear Feedback Shift Registers}

A linear feedback shift register is a finite state machine comprised of a unidirertionial chain of D-FF (unit delays) and XOR gates (modulo 2 adders). A lypical LFFil will XOR elements positioned between selected memory stages, is shown in figure 11

Such an LFSR generates a cyclic binary sequence by performing a linear tramsformatimn - polynomial division - on its input sequence (for move information see $\left.\left|\Gamma^{\prime} e t, 72\right|\left|n_{d 1} 87\right|\right)$ In implementing polynomial division, the feedback taps of an $n$ bit LFSR define lhe 


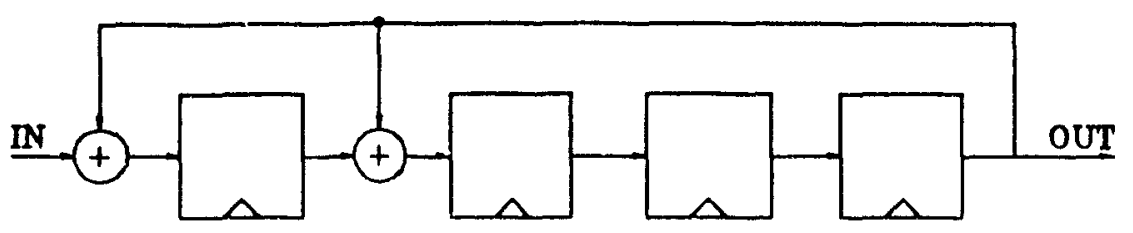

Figure 4.1 LFSR1 - polynomial divider - Characteristic Poly $r^{4}+r^{3}+1$

divisor or "characteristıc polynomial", and the input sequence (input polynomal) is the dividend. The $n$ bit contents ( or signature) of the unit after the last input bit has entered is the remainder polynomial and the sequence shifted out during the process a the quotient

A second LFSR structure is shown in figure 4.2. Here, a single XOR structure, positioned at the input of the first memory element of the LFSR, combines the previous states of seiected LFSR stages.

Both figure 4.1 and figure 4.2 share the same characteristic polynomial. $\left(x^{4}+x^{3}+1\right)$ and in terms of analysis, there exists a one to one mapping between the two LFill forms. LFSR1 is a true polynomial divider, and while both it and LFSR2 yield the same quotient stream, their respective signatures may differ.

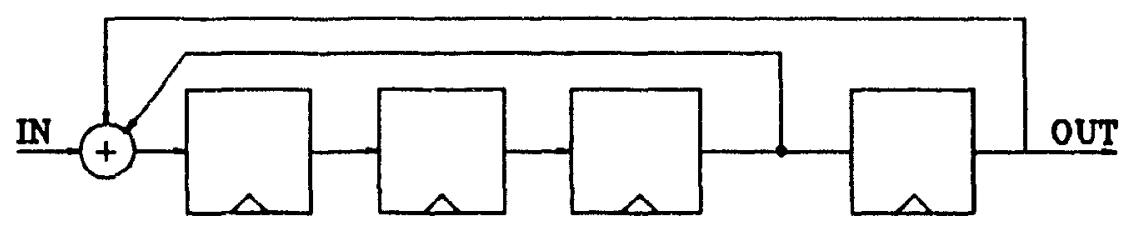

Figure 4.2 LFSR2 - Characteristic Poly $x^{4}+x^{3}+1$

An LFSR is said to be autonomous if there is no external input stream. For an n-stage autonomous structure, the number of unique states cycled through is determined bv the positions of the feedback taps.

If the characteristic polynomal is primitive ${ }^{4}$, the number of unıque internal states re-

4 A partial list of primitive polynomials can be found in [Bar87] 
peatedly cycled through is equal to $2^{n}-1^{5}$ In such a case the LFSR is said to be ol maximal length.

A property of maximal length LFSRs is that the distribution of output bits generaterl is uniformly biased to 0.5 (the ratio of 15 to 0 s occurning at edch memorv position one. the maximal sequence is $\frac{2^{n-1}}{2^{n-1}-1}$ ). This property and their relatively uncompliralon structure, make LFSRs an attractive base for pseudorandom number generators

\subsubsection{Cellular Automata}

Another sequential structure which exhibits pseudorandom (uniformly distributed) generating traits is the cellular automaton. The unit is a series of flip-flops where the communication is restricted to nearest neighbours. The structure and rommumication of each block is defined by a set of linear "rules" |Wol83| which deline the block's brhavior. Two common examples are blocks constructed rule 90 and rule 150 . (there aro 256 possible rules). They are shown below where $s[t]$ is the value of posthon/stato 1.11 time interval $t$ :

$$
\begin{aligned}
& \text { Rule 90: } \quad s[t+1]_{\imath}=s[t]_{\imath-1} \oplus s[t]_{\imath+1} \\
& \text { Rule 150. } s[t+1]_{\imath}=s[t]_{\imath-1} \oplus s[t]_{\imath+1} \oplus s[t]_{\imath}
\end{aligned}
$$

A hybrid CA is one which is constructed using more than rule.

[Glo88] [Hor88] [Ser88] have claimed that CA based generators possess superior randomness properties than LFSRs. In fact, [Ser88] establishes an isomorphism betwerll the two structures which tends to ease analysis since much theory has already beell developed for LFSRs.

\subsection{Test Response Evaluation}

There are many ways to analyze test responses. Conceptually. the simplest of these is lon externally compare the circuit output with the corresponding fault, free response fonml

5 The all zero state is excluded since it forms its own cycle 
through simulation As described in [Dav76], CUT operation may also be compared 1o that of a fault-free reference unit.

If test response evaluation is to be done on-chip or on-board, or if it is desired to reduce. by orders of magnituce, the amount of data transfer between the CUT and the test head. data compaction tec'iniques are employed. Compaction involves operating on the output data stream using some function which either performs a transformation or extract.s some qualitative feature of the output data, or both The most popular compartion scheme is signature analysis [Fro77] |Koe79] [Dav80| in which an LFSR or C $\Lambda$ pertom a polynomial division-like operation on the output stream. In this transformation, all possible input bit streams are mapped evenly onto the $2^{\prime \prime}-1$ possible sig ratures ol an $n$-bit signature analyzer. It follows that the number of $k$-bit input streams which produce the same signature is:

$$
\frac{2^{k}}{2^{n}}=2^{k-n}
$$

This raises an important point: since compaction is a reduction of information, there is an implied probability that useful knowledge is $\operatorname{lost}^{6}$ In the case of signature analysis using an $n$-bit signature analyzer, for every given fault-free signalure, there are potentially $2^{k-n}-1$ wrong bit sequences which could produre the same result The phenomenon where an incorrect bit sequence yields the fault-free signature is called aliasing. It has been found that as the length of the input sequence tends to infinity, an n-bit LFSR or CA alias with probability $2^{-n}$ [Wil86] [Iva88].

Typically LFSR based signature analysers accept serial data. The extension for multiplo output circuits is a multi-input signature register (MISR) which shown in ligure 4.3 lor a characteristic polynomial of $x^{4}+x^{3}+1$.

Other compaction techniques involve recognizing certain attributes of the circuit's output data stream, such as parity (Car82), the ratio of 1 's contamed in the stream and its extension to exhaustive testing (syndrome testing)[Sav80], and the number of transitions occurring [Hay;6]. Good overviews of various compaction techniques are given

6 compaction should not be confused with 'compression' in which all information is recoverable 
43 Built-In Self-Trat

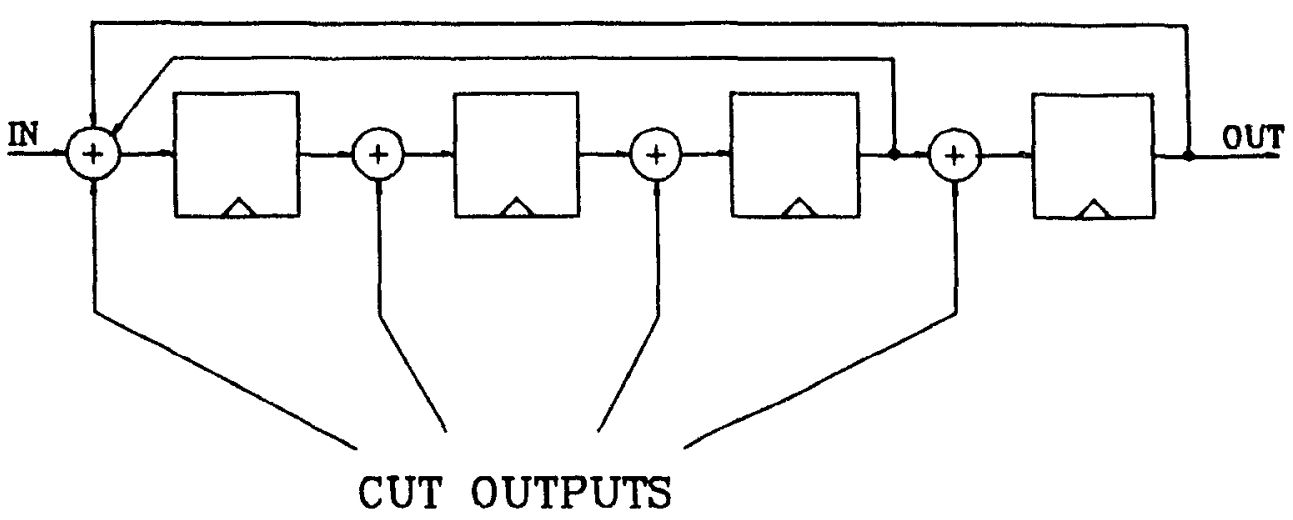

Figure 4.3 MISR $-x^{4}+x^{3}+1$

in $[\mathrm{Bar} 87]$ and $[\mathrm{Kar} 85]$.

\subsection{Built-In Self-Test}

In Built-In Self-Test (BIST), test generation and response evaluation hardware are IIcluded on-chip so that in-circuit tests can be performed with minimal noed of extornal test equipment, if any. The standard BIST set-up is shown in figure 4.4 Inder normal operating conditions, the additonal test circuitry is transparent to the lunctionality ol the device.

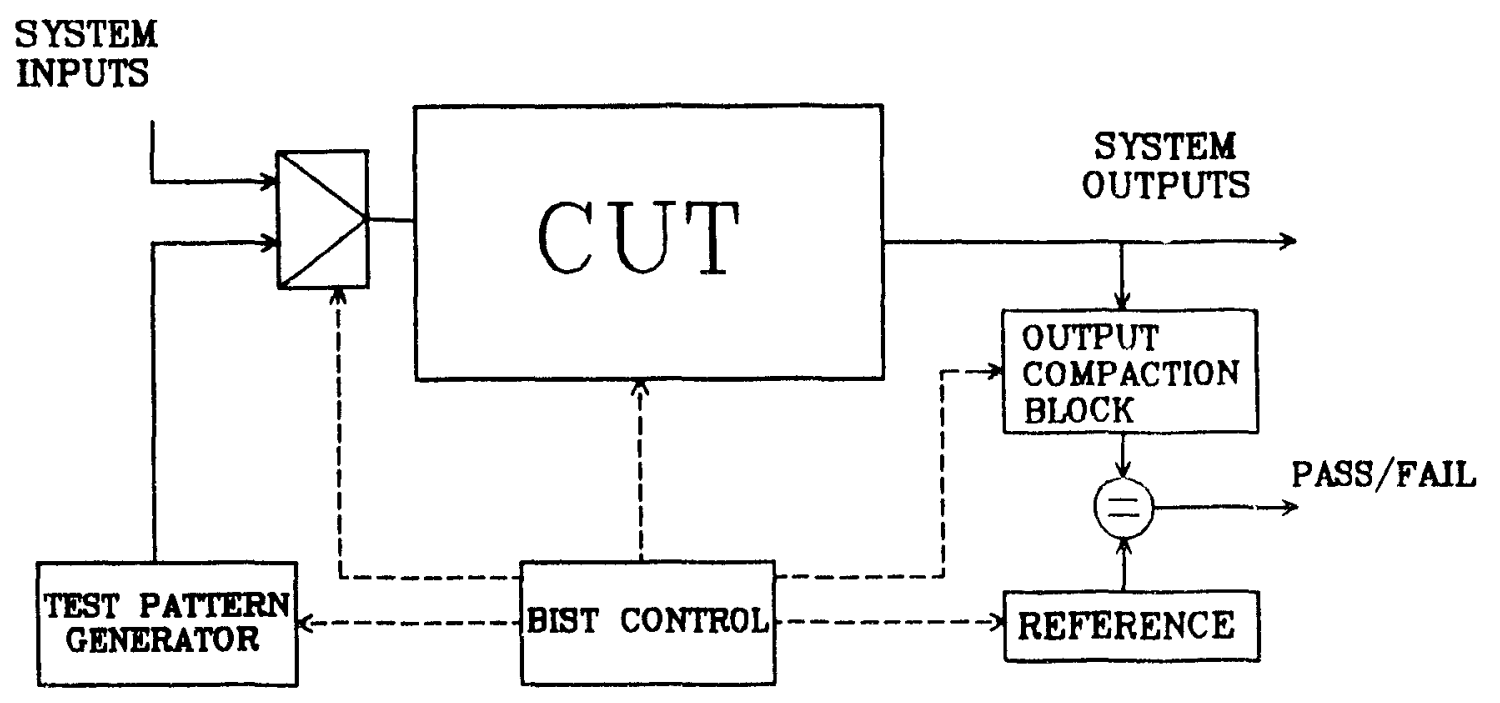

Figure 4.4 Standard BIST Sclieme 
During test mode, input stimuli are accepted from the test pattern generation blor $k$ (TPG) and the CUT's output responses prepared for analysis in the output compaction block (OCB) The most popular compartion technıque for BIST is signature analysıs At the end of the test session, the final signature(s) is / are compared with a referenc value(s) and a result flag is signaled. This type of process typically gives a global pass/fail diagnosis (if desired [Ast89] suggestis unclusion of more refined fault location features).

The reason for pursuing a self-test option is ultimately to reduce test costs, and their are, indeed, many potential advantages offered by such a strategy. For cxample :

- Less costly external test equipment is needed. thus the associated capital cost is reduced.

- Placing the application and verification corcuitrv withm the CUT eases the test access problem.

- Less dependency on physical leads incrcases the efficiency of testing at. other levels of packaging (e g. probe tests and board tests) |Bar87] [Par89|

- Shorter test times are possible since some tests can be run at circuit speerls [Kra87].

- Potentially lower (test) run-time costs because of possible circuit partitioning

- Increased portability of testing (espccially in-field test) allowing, lower long term maintenance costs (e.g user-initiated tests for microprocessors (Kub83!)

However, some of the obvious hurdles which thwart the acceptance of BIST are

- The extra area needed to implement the test circuitry reduc es device yield.

- There is a potential performance degradation if test circuits are included in a critical delay path.

- As with external pseudorandom testing, excessive generated test lengthis imply large time penalties.

- Common fault diagnosis techniques may be unattractive because, for example, constructing faut dictionaries for cincuits with compaction is $\mathrm{ex}-$ pensive |Ait89|.

- There is a general lack of knowledge and design techniques to solve the above problerns. 
Proponents of BIST claim that the long term benefits offered by this teat option lin outweigh the drawbacks - in some cases even if the area orerhead is $10 " 0$ "Tot $88 \mid$ Finthermore self-test methodologies may be the only solution to teat access problems which

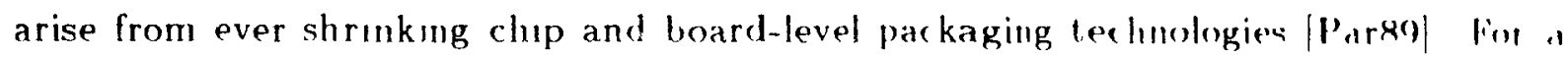
good introduction to BIST see [McC85a) [Mc(:85b) |Bar87]

Since this thesis presents an on-chip test pattern generation technique. the next section introduces two pattern gerierators used for BIST.

\subsubsection{Some BIST Test Pattern Generation Methods}

The TPG is usually implemented as a variation of simple random pattern generating structures, such as LFSRs or CAs, configured to generate a maximum length serfuence Sometimes, for example in the microcoded BIST of microprocessors, memors is includerl into the TPG Whether or not memory is included depends, however. on the amount al implementation overhead which can be tolerated.

One of the milestone structures proposed for BIST is a multifunction aheft register a alled a "built-in logic block observer" (BILBO) [Kon79] The BILBO, and lis cellular antomaron counterpart, CALBO (cellular automaton logic block observer (Hurg8|), facilitate partitioning the CUT into separate blocks for testing ln a particular lest session. Ilı unit acts as either a TPG or a signature analyzer (SA). Figure 4.5a is a tivpical BIGT set-up using a BILBO. Circuit olocks $A$ and $B$ are tested in separate fect, sessmill in the first, the BILBO acts as a compacter for circuit $\Lambda$, and in the secoml it ats as a pseudorandom TPG for B. In this case, since separate blocks share the IBII,IBO) for different purposes. the blocks $A$ and $B$ cannot be tested in parallel

In order to reduce the test time and hardware overhead in curred when implementuln a BILBO, some authors (e.g. [Kra87||Kim88]) s'ggest the use of highathe registers a TPGs. Such a system can increase parallelism when testing separate hlorks (provilor) there is no feedback between the blocks) This is demonstrated in Figure 45 , Will this setup, circuit blocks $A$ and $B$ can be tested in parallel because eac h intermerdial." 


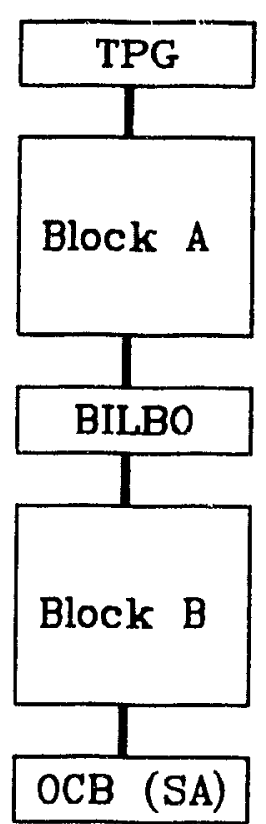

(a- Circuit Partitioring using BILBO)

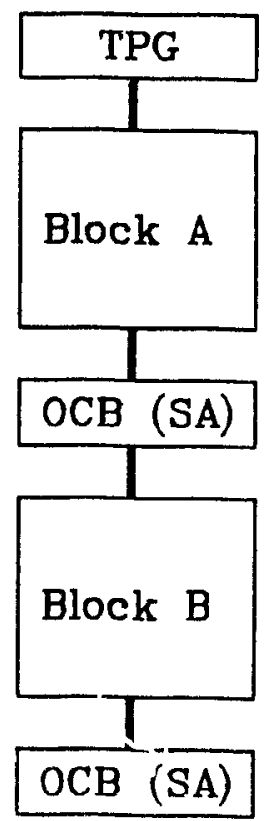

(b- Parallel testing of $A$ and $B$ )

Figure 4.5 BIST of Separate Circuit Blocks

signature provided by the OCB of A are used as test patterns for block B.

Circular BIST ([Kra87]) extends the idea of using signature registers for test pattern generation by creating a feedback shift register, where the CUT is the feedback logic, 1, simultancously generate and compact test patterns for the CUT itself. Somc altributes of this scheme are: tests can be run at system speeds, the entire circuit can be tested in one test session, and the overhead may be less than that for a standard BILBO scheme. In order to reduce the area penalty of implementing a full scan circular path. |Pra $88 \mid$ documents a method for selecting appropriate flip-flops for a partıal scan circular selftest path. The effects of phenomena such as fault masking should be evaluated in a circular BIST scheme. 


\section{Chapter 5}

\section{Testing With Weighted Random Patterns}

Conventional uniform random pattern generation, attempts to cover all faults willhin the CUT by generating a sequence in which there is an equal chance of each C'T'T inpul's assignment being 1 or 0 . Experimental results |Sch75| |Sav84||Lis87||Waik8||Wun88| [Bas89|, however, suggest that generating groups of test vectors such that there is : non-uniform distribution of 1's to 0's on the input lines, can result in test, lengths orders of magnitude shorter than that incurred when using uniform random pattern generalien alone. In such a generation strategy, the proportion of times an inpul. line assummen value of 1 is called the weight or bias of that line, and the complete distribution of biases for a group of CUT inputs is called a weight set or weight distribution Thus. according to the above definitions, a uniform random pattern generator possesses i characteristic weight set in which all pin biases are approximately equal to 05 t Test. pattern generation according to one or more non-uniform weight sets is a alled weighterd random pattern (WRP) generation.

This chapter is divided into two secions : First, some of the known algenillums used 1 " determine circuit specific weight sets are described; followed by a disc ussion of some of the current schemes used to implement. WRP generalion in handwat"

\subsection{Some Algorithms to Determine Weight Sets}

Unlike the new method proposed in this thesis, some documented methods usel I" 
calculate circuit specific weight sets involve one or more of the following techniques to gather information about the circuit function or structure correlation of internal switching activity to specific pin activity, formal detection probabilitv calculations, and circuit path tracing. The salient features of some of these methods are discussed below

In some earlier work intended for large-scale integration (LSI) proposed by Schnurmann et al. |Sch75|, the relative weight of a particular input pin is adaptively assigned based on the relative amount of internal switching activity caused by uniquely exercising that pin With respect to VLSI circuits, Siavoshi [Sia88] proposes an alternate adaptıve algorithm which reduces the prohibitive amount of simulation needed in [Schi55] to evaluate nodial activity. Instead of randomly toggling single input pins. most of the 1 initial information is gathered using a functional test set which alrearly exists for design verilic ation punposes By simulating each pattern of this set, the amount of activity per vertor is assessed from observing the circuit inputs in conjunction with the corresponding output states. This information is then used to develop an inttial weight set, from which weighted frst patterns are generated and simulated If any new test vectors are found as a result of WRP simulation, the estimated weight set is iteratively refined using a simılar proress A potential drawback of the method as presented is that a functional test set may not. always be a proper choice for the prime source of information, especially if it provides poor fault coverage. In such cases, the initial information may be biased resulting in the final test sequence being geared to test only a portion of the circuit.

Recently, Wunderlich [Wun88\} developed a detection probability baserl procedure to determine a user specified number, $k$, of weight sets. This is done by modifying the common test length formula (equation 1) to the form shown below (equation 2), which accommodates for $k$ generator weight distributions $X_{1} \ldots X_{k}$ and $k$ fault sets, $F_{1} \ldots F_{k}$.

$$
P(X) \leq \prod_{\imath=1}^{k} \prod_{f \in F_{\imath}}\left(1-\left(1-p_{f}\left(X_{\imath}\right)\right)^{N_{\imath}}\right)
$$


where

$$
N=\sum_{i=1}^{k} N_{\imath}
$$

If only $P$ is known, minimising the overall test length $N$ is an NP-harl task |Krik 4 | So, $k$ is made a free-variable and the new objective is to find a sufficintly small lest. sel. The problem is formulated as follows:

Given a probability $P$ that all faults are detected. let $k$ be the desired number of genetaton distributions Find $k$ partitions of the fault set $F=-F_{1} F_{k} \quad k$ associated weight distributions $\mathrm{X}=<X_{1} \quad X_{k}$ > and $k$ test lengths $=N_{1} \quad N_{k}$. such that the total leat length $N$ is sufficiently smaller than that of a unform random test

The procedure for determining multiple weight sets is an extension of the work contiainm in [Wun87] which solves the case of a single weight set.

\section{Single Weight Assumption}

For a single fault set (i.e. $k=1$ ), an optimal solution for $X$ can be found by minimicing the objective function $(O F)$ -

$$
\begin{gathered}
\ln (P(X)) \approx \sum_{f \in F}-\left(1-p_{f}(X)\right)^{N} \approx-\sum_{f \subseteq F} e^{-p_{f}(X) N} \\
O F=\sum_{f \in F} e^{-p_{f}(X) N}
\end{gathered}
$$

but this task generally requires exponential effort.

However, since the $O F$ can be proven to be strictly convex with respect to a single variable $^{7}$, heuristically, a suitable distribution $X$ can be found using Newton Iteratinn (or similar method e.g regula falsi) to minimise the $O F$ for each pin bias $x_{1}$.

\section{Multiple Weights}

The procedure for developing multiple weight sets involves partitioning the fault sct. $F$ into $k$ subsets $<F_{1} \ldots F_{k}>$ of possibly diflering sizes, such that the sum of thr $k$

7 Note that $P(X)$ is really a function of $m$ variables since the weight $X$ is a set of input biases . $r_{1}{ }^{5} m$. wher" $m=$ no CUT inputs 
51 Some Algorithms to Determune Weight Sels

associated $O F \mathbf{s}$ is sufficiently small. A weight is calculated for each separate fault grouping.

Again, the problem of finding an optimal division of the fault set requires exponential effort Instead. a multi-stage partitioning scheme is adopted. First, a subset of fault.s with the smallest detection probablities (see [Sav84]) is coarsly partitioned into $k$ groups using a first order algorithm afterwhich the contents of these $k$ fault groups then refined using a search tree. Each remanning fault is then assigned to one of these subsets. A complete description of the method is contained in |Wun88|.

\begin{tabular}{|c|c|c|c|c|c|c|c|}
\hline & \multicolumn{2}{|c|}{ ESTIMATED LENGTHS } & \multicolumn{5}{|c|}{ SIMULATED LENGTIIS } \\
\hline \multirow[t]{2}{*}{ NETLIST } & \multirow{2}{*}{$\begin{array}{l}\text { Random } \\
\mid \text { Wuns8| }\end{array}$} & \multirow{2}{*}{$\begin{array}{l}\text { Weighted } \\
\mid \text { Wun88 } \mid\end{array}$} & \multirow{2}{*}{\multicolumn{2}{|c|}{$\begin{array}{l}\text { Weighted [Wun8s] } \\
\text { Test len. \# Sets }\end{array}$}} & \multirow{2}{*}{\multicolumn{3}{|c|}{$\begin{array}{c}\text { Random } \\
\text { (Tulip) }\end{array}$}} \\
\hline & & & & & & & \\
\hline $\mathrm{C}, 432$ & $1.9 \mathrm{e} 3$ & $11 \mathrm{e} 3$ & 494 & 2 & 1088 & 608 & 1021 \\
\hline C:199 & $1.2 \mathrm{e} 3$ & $1.2 \mathrm{e} 3$ & 1.381 & 1 & 960 & 1216 & 1600 \\
\hline $\mathrm{C} 880$ & $2.4 \mathrm{e} 4$ & 710 & 578 & 3 & 21568 & 6848 & 9760 \\
\hline$(: 1355$ & 2106 & 2106 & 5288 & 1 & 3104 & 37,14 & 1824 \\
\hline C1908 & $5.1 \mathrm{e}$ & $2.1 \mathrm{e} 4$ & 1074 & 6 & 10592 & 6if88 & 15072 \\
\hline $\mathrm{C} 2670$ & 8806 & $8.1 \mathrm{e} 5$ & 47110 & 5 & $3.7 \times 6$ & 4306 & 6 1 1 6 \\
\hline $6 ; 3540$ & $8.0 \mathrm{e} 5$ & $1.1 \mathrm{e} 5$ & 11233 & 5 & 49248 & 84416 & 9280 \\
\hline C5315 & $4.0 \mathrm{e} 4$ & $1.1 e 4$ & 4430 & 5 & 1920 & 2656 & 1128 \\
\hline C6288 & $6.3 \mathrm{e} 2$ & $2.4 \mathrm{e} 2$ & 239 & 1 & 128 & 160 & $i 92$ \\
\hline C7552 & $3.1 \mathrm{e} 11$ & $2.8 \mathrm{e} 5$ & 240.37 & 6 & $\Rightarrow \ln 6$ & lef & 106 \\
\hline
\end{tabular}

Table 5.1 Comparison of [Wun88] Data to Random Pattern Simulation Results

Based on the results contained in $\left|W_{u n} 88\right|$, the performance of this methorl is dillicult to evaluate. Some of the data is reproduced in table $51^{8}$ Here, the estimated uniform random test length and the estimated 'optimal' weighted test length are shown against the number of simulated weighted patterns|Wun88| and three uniform random t.est lengths found via simulation (Tulip (Maa88)).

8 For C7552, after weighted simulation 27 faults were left undetected 
The following observations are made :

o Compared to the simulated random test lengths, the equiprohahle test length estimates seem excessive. In fact, in all but two cases (c2070) and C7552) the gain of using the determined weight sets is not significant This is possibly because the estmmates attempt to give a conficlence level that some large percentage (eg 99\%) of all test scruences of this lenglh will achieve $100 \%$ coverage

(2) The weighted test length estimates for 4(6:1908, C:1355, (:2670) and (:75:52) cases differ trom the weighted simulation value by at loast an order of magnitude

P The major advantage of using WRP is to diminish the fost length nerderl to achieve a high level of fault coverage Aside trom (2670) and (:7552, all of the ISCAS85 [ISC85) test circuits are fully lestable an a relatively short pseudorandom test. length thus don't benefit from the sheme The weighting algorithm of [Wun88] could be better evaluated if expentume were performed on circuits which require much longer random tes: lengths

Also, contrary to what is logically expected, it is found that when using this method, the test length does NOT monotonically decrease with an mcreasing number of determmerl weight distributions This in itself is a serious failure on the part of the algorithm The author attributes this to the inherent complexity of the problem and the approximation:; used in the applied heuristics The method's sensitivity to detection probability arcuracy and the size and contents of the initial fault list may be another sounc of ertor. For example, if the contribution of redundant faults is not neglecterl, the calculated detertion probabilities of this class of fault would corrupt intermediate test length estimatess and result in inaccurate weight sets and a pessimistic overall teest length projection

Bardell, Savir and McAnney [Bar87] discuss a path tracing algorithm fo find an InIl lal weight set. This is done based on the signal probability assignment to the inputs of a gate, and calculated according to the formulae given below Here, $p$, is the bias ol the inputs to the gate, $p_{0}$ is the output signal probability, and $k$ is the number of gate inputs.

The weight of a fanout stem is calculated as the mean of the signal probability assign- 


\begin{tabular}{|c|c|}
\hline BLOCK & $p_{\imath}$ \\
\hline AND & $p_{o}^{1 / k}$ \\
\hline OR & $1-\left(1-p_{0}\right)^{1 / k}$ \\
\hline INV. & $1-p_{0}$ \\
\hline NAND & $\left(1-p_{0}\right)^{1 / k}$ \\
\hline NOR & $1-p_{0}^{1 / k}$ \\
\hline
\end{tabular}

Table 5.2 Backtrace Signal Probability Update Formulae [Bar87]

ments at each of its branches This, however, may not always be the most approprialn compromise because the relative "importance" of each branch is not considered. For instance, due to the averaging process, the contribution of a branch bias intended to cover a relatively large number of circuit faults, may be outweighed or cancelled (the average reverts to 0.5 ) by the bias(es) of one or more other branches, whose cumulative number of associated faults is far less than that of the previous branch $\Lambda$ solution might be to rank each branch according to the size of the sub-circuit, thus the number of circuit faults, with which it is assocrated. The bias of a stem can than be estimatrol as a weighted average of the biases at each of its branches.

The weighting procedure of $[\mathrm{Bar} 87]$ is as follows :

For each gate in the circuit, an initial weight is assigned to each of its $n$ inputs according to the formulae.

$$
\begin{gathered}
A N D, N A N D: p_{\imath}=(n-1) / n \\
\text { OR,NOR: } p_{\imath}=1 / n
\end{gathered}
$$

and the gate's contribution to the signal probability assignments at the CU'T inpuls is computed by propagating back the gate's output signal assignment along all possible backtrace paths, and updating according to the equations outlined in table 5.2.

As a result, after all gates are processed, each CUT input will have $m$ assigned local weights, where $m$ is the number of possible gates which can be traced to that CUT input. The bias of a CUT input is then calculated as the average of all assigned local 
51 Some Algorithms to Determine Weight Scte

weights. See $[$ Bar87] for an example of this.

Multiple weight sets are found in the following manner: Using the initial weight set calculated above, a threshold number of weighted lest vectors are simulated. The fallis left undetected after sequence are identified. ATPG is performed to determine a teal vector for one of these remaining faults and a test weight is computed according to the form of this new vector. That is, for each logical 1 in the test, a sulficiently high signal probability (e.g. 0.9) is assigned to that input position. Similarly, mput positions corresponding to 0 values are assigned a low weight (e.g. 0 1) Sio, the test vector ly yo()I may produce the weight $(0.9,0.5,0.5,0.1,0.1,0.9)$. Again, simulation is performed and the fault list is further reduced. The ATPG-simulation based procerlure repeats lo fiml other deterministic weights ${ }^{9}$.

An intermediate procedure which can be used in conjunction with the $\triangle T P(i$ ste to find multıple weights is to iteratively perform the initial sıgnal probability based weighting algorithm on progressively reduced fault sets. Thus, dedicated weights will be algorithmically found for faulty 'sections' of the CUT This would be beneficial if weights defined by different subcircuits conflict, and tend to 'smooth' parts of the final weight set back to a near uniform random distribution.

An algorithm which uses such an idea is proposed by [Lis87]. Test generation system ESPRIT ( Enhanced Statistical PRoduction of Test vectors) is designed to use lestiability measures and feedback form fault simulation to dynamically generate test vectors according to multiple weights

In this approach, testability estimates $[\mathrm{Brg} 84 \mid$ are used to calculate input probabilit,m by minimising a cost function which balances CPU time and simulaterl test length Patterns are generated according to the assigned weight set and fault cimmlation 14 done until a threshold rate of fault coverage is reached. Using the resulting reducorl

9 In such methods, it is always an option to store a few test patterns rather than implement a pure weighted random test strategy 
undetected fault list, (thus for simulation purposes, a reduced representation of the circuit) the process repeats to find a supplemental weight distribution This procedure of determining weights and updating the fault list continues until a threshold ievel of fault coverage is attained

Experimentally, compared to a random pattern test length. the scheme is found to successfully diminish the generated test length needed to test the ISCAS85 benchmaık circuits[ISC85). The results of $\mid$ Lis87| did not, however, discuss the number of weight distributions computed during the procedure

In an algorithm patented by IBM $[\mathrm{Eic} 87]$, all possible paths from an output terminal to an CUT input are traced and an initial weight is calculated as a function of the number of CUT inputs feeding the blocks along the trace-back paths. Weighted simulation is then peiformed and DTPG is used to find test vectors for any faults left undetected Additional weight sets are derived from the DTPG results. The procedure for estimating the initial weight is discussed below :

In this algorithm, each input of a circuit block, $X$, shares the same weight value The individual device blocks are limited to AND, NAND, OR and NOR primitives. Assuming that identical circuitry feeds each of a block's $N$ inputs, the probability, $P$. of placing a non-controlling value on any block input is approximated by (no justification for this was given in (Eic87]):

$$
P_{m i n}=\left(3-N-\left(N^{2}-2 N+5\right)^{1 / 2}\right) /(2-2 N)
$$

It follows that the ratio of occurrence of a non-controlling setting to controlling assignment is

$$
R_{m i n}=P_{m i n} /\left(1-P_{m i n}\right)
$$

For example, in order to minimise the generated test length needed to test all stuck-at faults on a 4 input OR, the probability of placing a 0 on each gate input should be 0.768. In accordance with the definition of weighted generation given previously, the bias of each input line should then be 0.232 . 
Since identical input circuitry is a rare occurrence, a compensalion factor in int rolluc (..1 based on the number of CUT inputs which can be traced to the biock $r$ This in calculated as :

$$
K=0.5\left(N D I_{x,} / N D I_{y}\right)+R_{m n}\left(N_{r}\right)
$$

where,

$N D I_{r}=$ No. of CUT inputs controlling $X$

$N D I_{y}=$ No. of $\mathrm{CU}^{\prime} \Gamma$ inputs controlling the block $Y^{r}$ which feeds $\mathrm{X}$ along the selected traceback path

$N_{x}=$ No. inputs to $X$

Equation (11) can be interpreted as the ratio of non-controlling to controlling inpul probability being offset by the ratio of the number of CUT Inputs feerling the somun" block $X$, versus the number of CUT inputs feeding the block (Y) whirh is inpul In I

Each circuit block is assigned two numbers $W 1$ and $W 0$, which represent. the 1 and weights shared by all of the block's input lines. These values are illitıalized lo 1. anil due to the different functionality of the 4 block types, they are conditionally updaterl as in table 5.3.

\begin{tabular}{|c|c|c|}
\hline BL,OCK & $W 0$ & $W 1$ \\
\hline AND & $W 0_{x}$ & $K / W 1_{x}$ \\
\hline NAND & $W 1_{x}$ & $K / W 0_{x}$ \\
\hline OR & $K * W 0_{x}$ & $W 1_{x}$ \\
\hline NOR & $K+W 1_{s}$ & $W 0_{r}$ \\
\hline
\end{tabular}

Table 5.3 Weight Number(W'1.W0) Update Calculation |Eic 87|

Starting from each CUT output, a recursive backtrace is performed, pogsessively "1" dating the $W 1$ s and $W 0$ s of the input blocks encountered along the larktrace pathe The W1(W0) of an input block, $Y$, is modified as the larger of the W1(IO) value ale 11 . lated from table 5.3, and the $W 1(W 0)$ already assigned to the input block for axtmplr if block $X$ has a NAND as an input circuit block, the Ws for the NAND ( $i$ e blok 
$Y)$ are updated as :

$$
\begin{gathered}
W 0_{y}^{\prime}=M A X\left(W 1_{x}, W 0 y\right) \\
W 1_{y}^{\prime}=M A X\left(K / W 0_{x}, W 1_{y}\right)
\end{gathered}
$$

The backtrace then continues from the NAND. When an CU'T input is reached, its bias is indicated by the ratio of the $W 1$ and $W 0$ assigned to the line. For example if $W 0$ $W 1$ then the line is more strongly biased towards 0 . The reader is referted to $\mid$ Eic $87 \mid$ for further details.

Note that the compensation (equation. 11) is not accurate for circuits with fanout. and/or reconvergence. The root of the problem is the assignnent of identical W1 and W0 values to each of a block's input lines.

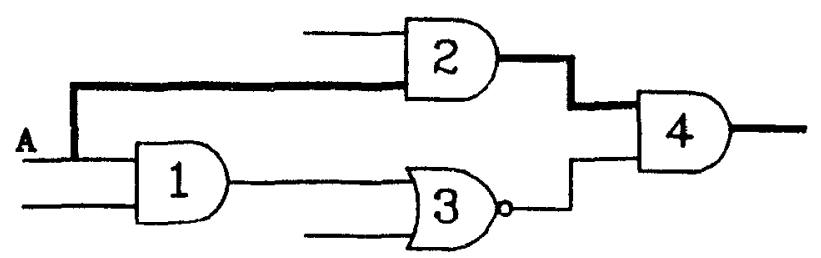

(i)

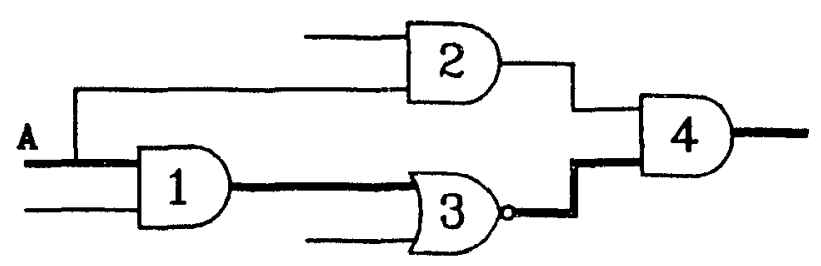

(ii)

Figure 5.1 Failure of [Eic87] due to Fanout

If a particular circuit line fans-out, then it is shared by more than one subcircuit and hence, more than one backtrace path. It follows that the weight assignment of the fanoul, stem dictated by each backtrace path may be different. Figure 5.1 is an illustration of 
this. The fanout stem $A$ is shared by gates 1 and 2 , thus the calculated W' for stem 1 may be conflicting depending on which of the backtrace paths shown is taken.

Regardless of the inherent inaccuracies of the respective weight estimation proresses. the acceptable performance of weighted test patten in generaton suggeste that the weight sets do not have to be optimal. In a practical sense, since thousands of test patierns an generated, the weight must simply represent enough information so that its performanc" is sufficiently superior to uniform random pattern testing

\subsection{Weighted Generators}

This section outlines some of the known implementation schemes used to realize WIR generation in hardware. Depending on how input stimuli are mapped onto circuit inputs. hardware WRP generators can be divided into two classes of architecture

- Local generators, and

- Global generators

\subsubsection{Local Generators}

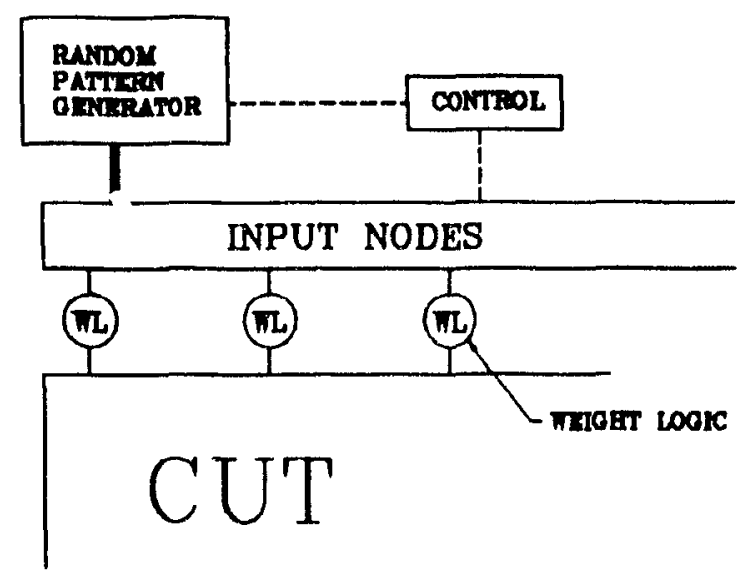

Figure 5.2 Local Weiglited Pattern Generation

In local generation, weighted inputs are computed and applied directly at an input, norls 
by logic associated with that node (figure 5.2). Since weight conversions are performed at the input sites, the hardvare overhead for this type of architecture tends to grow as a function of the number of weighted CUT inputs required. This implies that the weight. logic assigned to each CUT input should be simple. Moreover, the size and complexity of hardware required to locally generate weighted patten ns according to multiple nonuniform weight sets may be unacceptable for implementation

Some of the earliest wo-k in external local weighted pattern generation is containerl in $[$ Sch $75 \mid$ and reproduced in figure 5.3. Here, the weight at a CUT input reflects the relative amount of switching it undergoes. This however, does not detract trom the previous definition of weighted generation given at the begining of this chapter

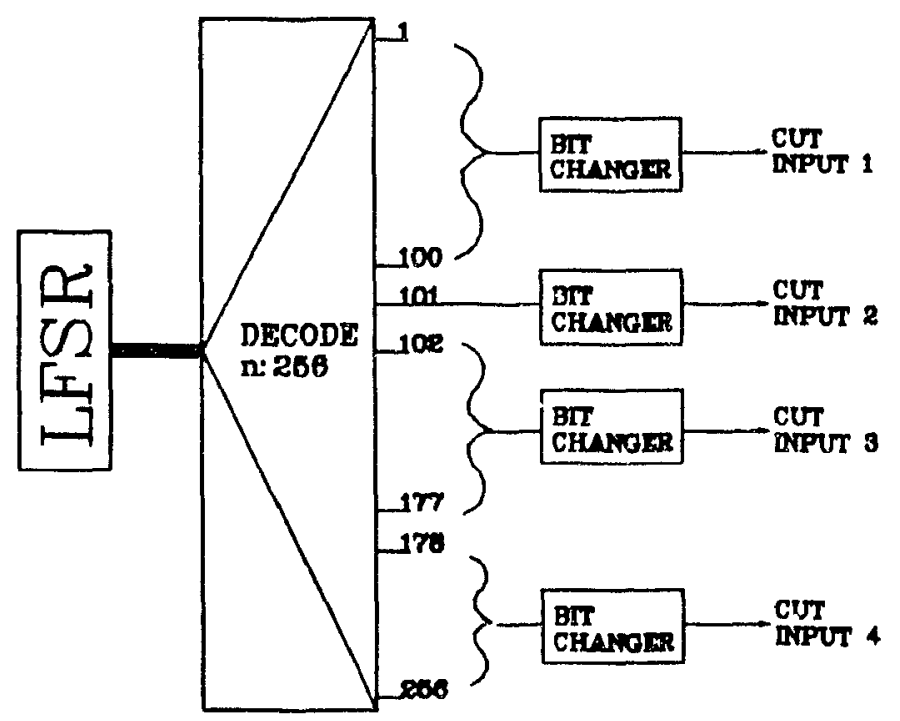

Figure 5.3 Weighted Gellerator of $[5 \operatorname{ch} 75]$

An LFSR is used to stimulate each input of a large decoder a uniform number of limes Each generator output is driven by a "bit changer" or binarv trigger. which toggles each time a signal is applied to it Thus, a relative weight, is assigned by diving a r:IIT input's bit trigger by a bundle of (ORed) decoder outputs. For example in figure 5 ,3,

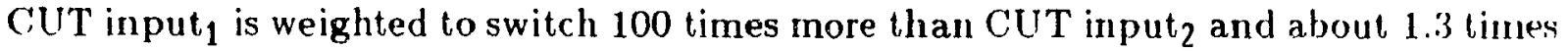
more than CUT input 3 and CUT input 4 . 
This structure is actually a good example of how technology change: impact test harrware. The technique may have been appropriate for circuits of the mid-70) (the test circuits in $[S c h 75 \mid$ contanned less than 50 inputs) but nowadays, the comparatively large number of circuit inputs resulting from techniques such as scan design, and the associated serial manner in which most of these CITT inputs are arcessed, renders this generation scheme infeasible. Incorporating the generator on-chip is impractical dur to the high degree of routing necessary.

\subsubsection{Global Weighted Pattern Generators}

The main characteristic of global WRP generators (figure 5.4) is that weighted hit streams are mapped onto the appropriate CUT inputs. Frequently, the actual patiern generation circuit is autonomous to the $U^{\top} T$ and a control block is needed to perlorm the mapping An advantage of this architecture is that the control blork can be constructed to enable test pattern generation according to multiple weight sets. In such cases, however, the size of this block may make the global WRP' generator unattractive for full on-chip implementation.

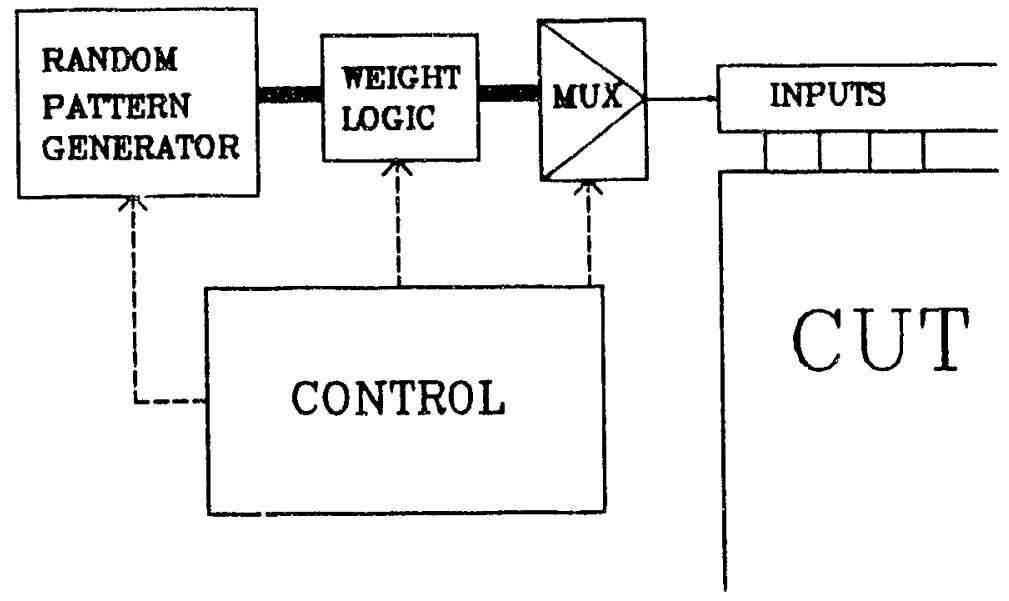

Figure 5.4 Global Weighted Pattern Generation

[Fed86] uses a memory based global WRP generator for the testing of microprocessors 
First, a feedback shift register is designed such that there is no correlation between subsequent states. As shown in figure 5.5, the uniform output of this n-bit. FSR is decoded to non-uniformly address a store (ROM) of $k$ instruction words, where $2^{\prime \prime}$ is greater than $k$ Thus, complete instruction patterns (not bits) are weighted by mapping a desired number of FSR states to that pattern's address in the ROM. The mothod is well suited to off-chıp functional testing but the ROM is may be too large for inclusion on-chip.

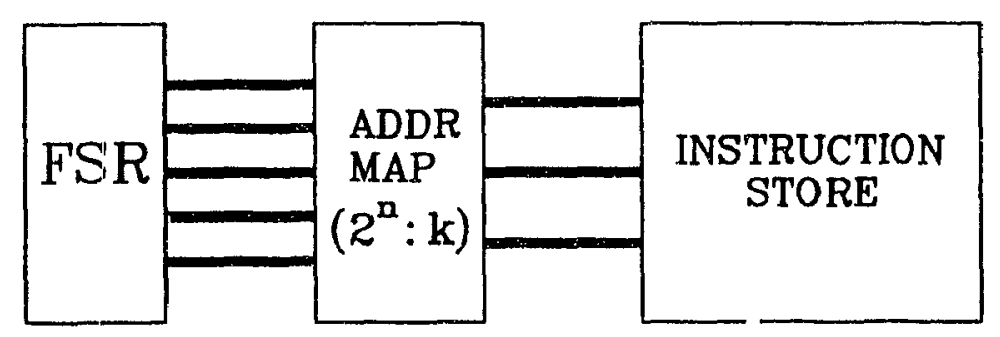

Figure 5.5 Weighted Instruction Generator

[Wun87] suggests a generator of unequiprobable random tests or GURT. This mullifunction register unit has 4 modes of operation -

- normal system operation wherein each register serves as a D-fiip-flop.

- a shift register

- a signature analyser

- a weighted generator

Of interest to this text is the last mode.

As a weighted generator, each GURT is configured to generate a 1 according to throc probabilities $p, 1-p$ and 0.5 . This is done in a manner similar to combining the random bits contained in distinct LFSR stages through a small combinational block, the ontpul. of which is brased to the desired weight In some cases, in order to amplement differnit weights (eg. 1/4 and 1/16) multiple GURTs are constructed $A$ major drawback of this test architecture lies in the mapping of the weighted stream(s) onto circuit inputs.

Since a GURT can only generate 3 weights, input (scan) nodes of similar bias must, be grouped to minımise ronting costs and avoud repeatedly constructung CiURTs of thr same weight. However, weight sets are usually developed after the CUT is designed and 
the resulting assignment of bias levels to CUT inputs can be in anv order. P'ost-design reordering of scan cells to regularize the weight assignments is not a simple task anl can (and probably will) introduce a severe routing overhead. Replicating (IIR's of Ilie' same weight may also introduce an unreasonable area penalty

|Eic87| describes a weight generation system which is integrated into an external lestor The basic weighting circuit is shown in figure 5.6 WRP gencration is based on combining (ANDing) the random bits output from up to 5 consecutive stages of a :32-bil. LFSR.

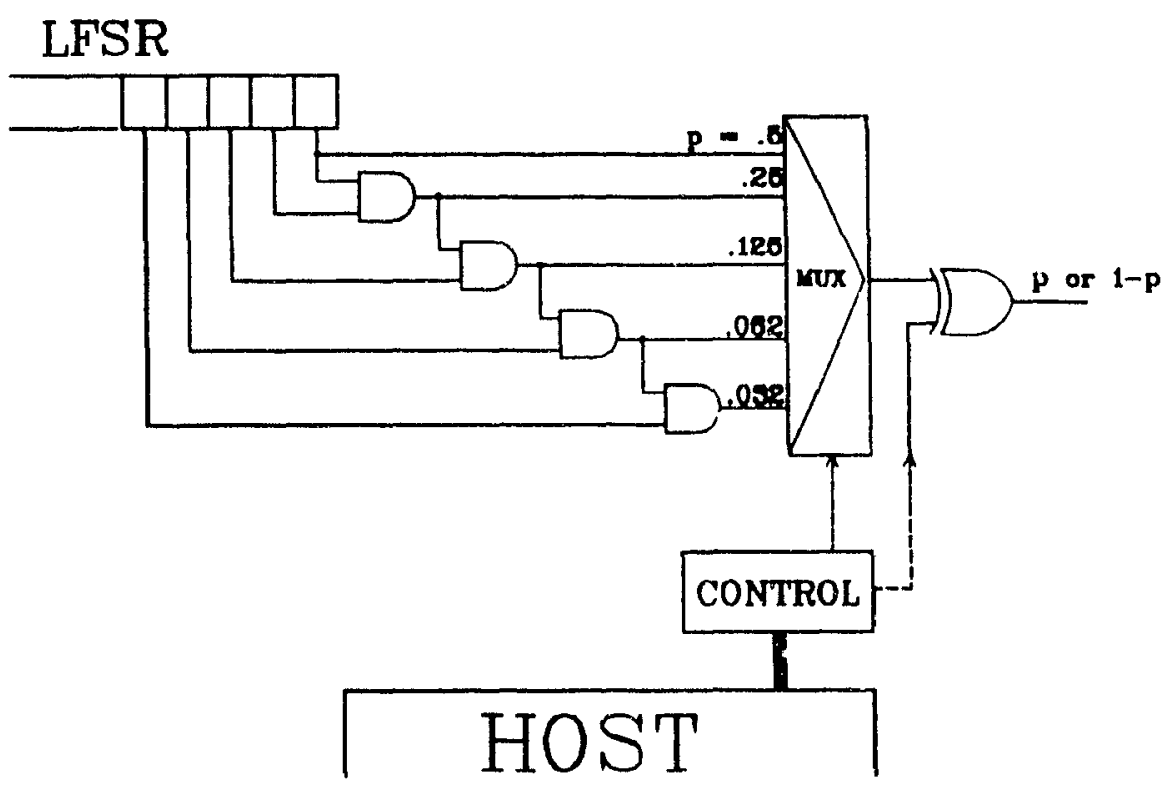

Figure 5.6 Cascade AND Weight System

Stream biases of 5 bit resolution are possible. The cascaded $A N D$ strurture rompules biases of $2^{-\imath}, \imath=1 \ldots 5$. The output XOR is used to logically invert the generated stream thus provide complementary biases of $1-2^{-1}$ for the same range of

In order to reduce interdependencies within the generated bit at ream, a delay-shift method of generation is used to stagger the spatial rate at which bits are combined For instance, if a bias of 05 is required, line \#1 of the output mux would be solentorl every shift cycle. On the other hand, because a bias of 0.125 is the product of $\Lambda$ NI)ing 
3 consecutive LFSR stages, when needed, line \#3 is gated through the MUX only after every 3rd cycle. The other non-equiprobable biases are generated in a similar manner.

Each output channel of the external test unit has a dedicated weight circuit, the LF, SI of which has unique feedback taps and a unique initial seed This is done to help ensure that a pair of independent device inputs do not repeatedly receive identical test stimuli The sizable amount of control information needed to select and map biases onto inpul pins is handled by an external host computer.

Another global patten generation system based on a "canonical weighting circuit" is suggested by $|\operatorname{Brg} 89|$ and shown in figure 57 The weighting circuit, is a cascade of , multiplexers whose select lines are determined by $r$ distinct taps of a maximal length pseudorandom source biased to 0.5. Dedicated to each of scan input. (s, ) of the $(: T T$ is a control word $\left(W^{r} W^{0} \ldots W^{r-1}\right)$ which is input to the multiplexer bock and designates the weight of the scan cell according to :

$$
W e i g h t\left(S_{\jmath}\right)=: 2^{-r} W^{r}+\sum_{\imath=1}^{r} 2^{-\imath} W^{r-\imath}
$$

In this way the unit generates patterns with a weight resolution accuracy of $2^{-r}$. The reader is referred to $[\mathrm{Brg} 89]$ for a detailed discussion of the weight derivation.

The size memory control block may tend to prevent the entire system from being integrated on-chip, however, on-board implementation is possible. The memory is expecterl to grow as $O(k n r)$, where :

$$
\begin{aligned}
& -k=\text { number of weight sets } \\
& -n=\text { number of scan cells. }
\end{aligned}
$$

Thus, chips with the order of 1000 scan inputs and 2 weight sets of precisior $2^{4}$, requirc the order of $10 \mathrm{~K}$ bits of storage. Since test time can be traded for hardware complexity, a more coarse weight set may be chosen in order to reduce the size of the memory.

The design of a new on-chip local WRP generation mechanism is presented and evaluated in the next chapter. 


\section{RANDOY PATTERN GENERATOR}

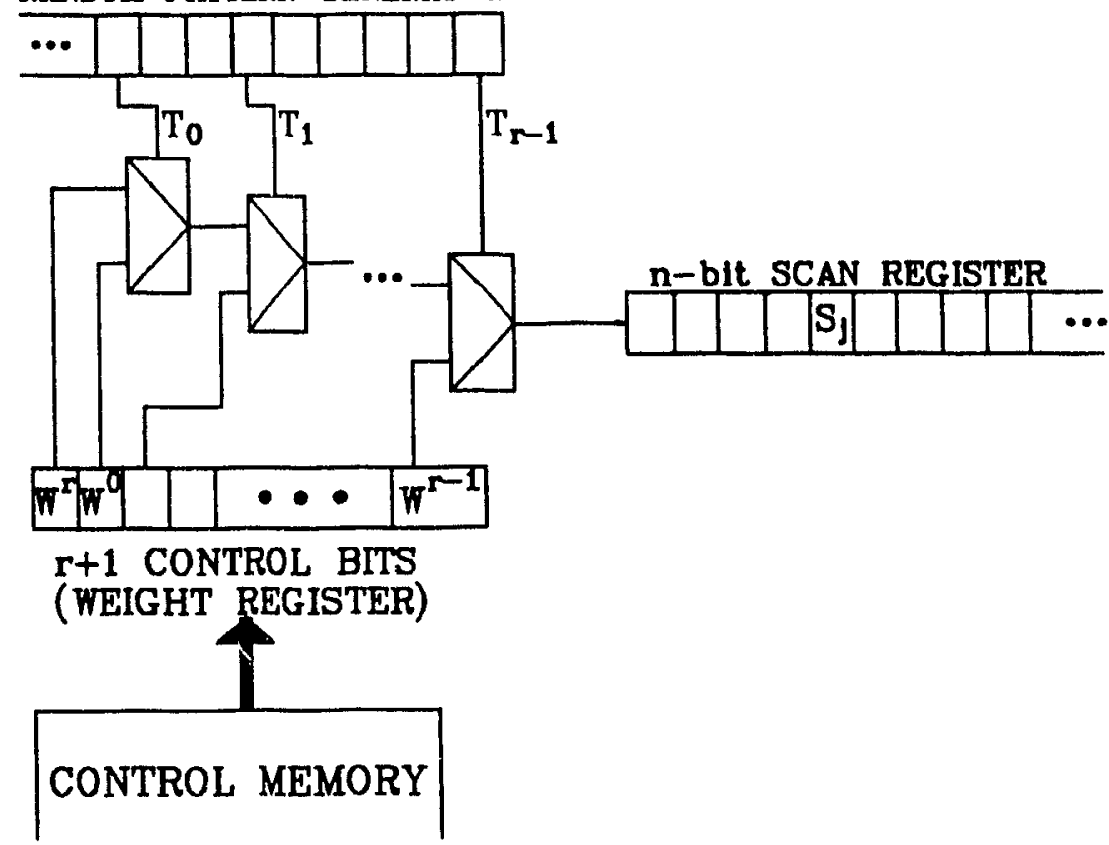

Figure 5.7 Canonical Weighting Circuit 
This chapter examines a BIST implementation of a WRP generalor for full serial scallpath (hundreds to thousands of scan cells) circuits. Previously it has been suggesterl [Wun88] [Wai88] [Lis87] that multiple weight sets can be used to minimize the overall test length, however in a BIST environment, the high cost of implementing different weight distributions must be considered. For this reason, test pattern generation is performed using only one uniform random weight set and one non-uniform weight set. of fairly coarse resolution. Experimental results will demonstiate that the peiformance of such a strategy is orders of magnitude better than uniform random pattern testing alone

Thie self-test methodology involves serially scanning in a test pattern and scanning out the corresponding test response into a compaction unit. Using such an approach makes the suggested BIST solution extendible to external testing. Experiments arr performed using the single stuck-at fault model, however, the analysis can be applierl to any fault model which does rot require a specific ordering of input, test patterns le.g non-feedback bridging faults, multiple stuck-at. etc.)

The text is divided into two sections: first, an ad-hoc simulation based method of determining the characteristic weight sets is outlined; and second, a distributed WRP generator is proposed for local generation of the dually weighted sequence. This generator design is intended for BIST and is suitable for automated design. At various 
stages, performance results are given in order to help demonstrate the effertiveness ol the procedure.

\subsection{Weight Set Estimation}

Much work has been done concerning the off-line development of compact test seta and sequences (chapter 2), but on-chip test application schemes are rare (recall lhal on-chip storage requirements of even a fraction of the test set mav be loo large [Bask(1) and unrealistic test lengths may be incurred when using only uniform inndom palton genertion). The test pattern generation scheme discussed here is geared for officient (in terms of time, storage and implementation) test application. It can be considered: means of transforming a conventional randomly generated test sequence into a pair al relatively short test sequences, one of which is non-unformly woighted Conceptualls. the approach is similar to the joint random pattern and stored pattern testing scheme which was described prevously in section 412 , except that in this race, stened palterll generation is replaced by WRP generation The rest of this section focuses on the dragin of the non-uniform weight set.

Unlike much of the previous work (Chapter 5), the weight estimation procedure presented relies neither on formal signal probability calculations nor on explicit analysı of circuit structure. As mentioned in chapter 5, in some methods which depend on such details (eg. [Bar87][Eic87]), fanout may dirertly affect the accurar of the final weight set. because conflicting woight assignments may be assigned to separate cim nil nets which converge at the same gate. Also, if the weighting algorithm is such that " detectability value for each potential fault contributes to the resull. unat probahilit estimates for redundant fauit sites may pollute the weight computation

In the work at hand, the conjecture is that sufficient circuit information pertinent In designing a weight set is contained in a test set, and can be extracted using simulation techniques. In fact, since the weight estimation process is based on the circuit's alm 
ulated behaviour during test mode, the circuit can be considered a functional "black box" during the procedure. Because fanout and redundant faults are not explicilly considered in a functional circuit reptesentation, they should not disectly contribute to the arcuracy of the final weight set. The existence of fanout and redundancies rlo, as usual, impact the computational effort of simulation tasks performed.

As an aid to the ensuing discussion, a flowchart of the entire scheme is provider in figure 6.1. The alphabetic tags associated with the various steps are refered to in the subsection(s) dcaling with that stage of the process.

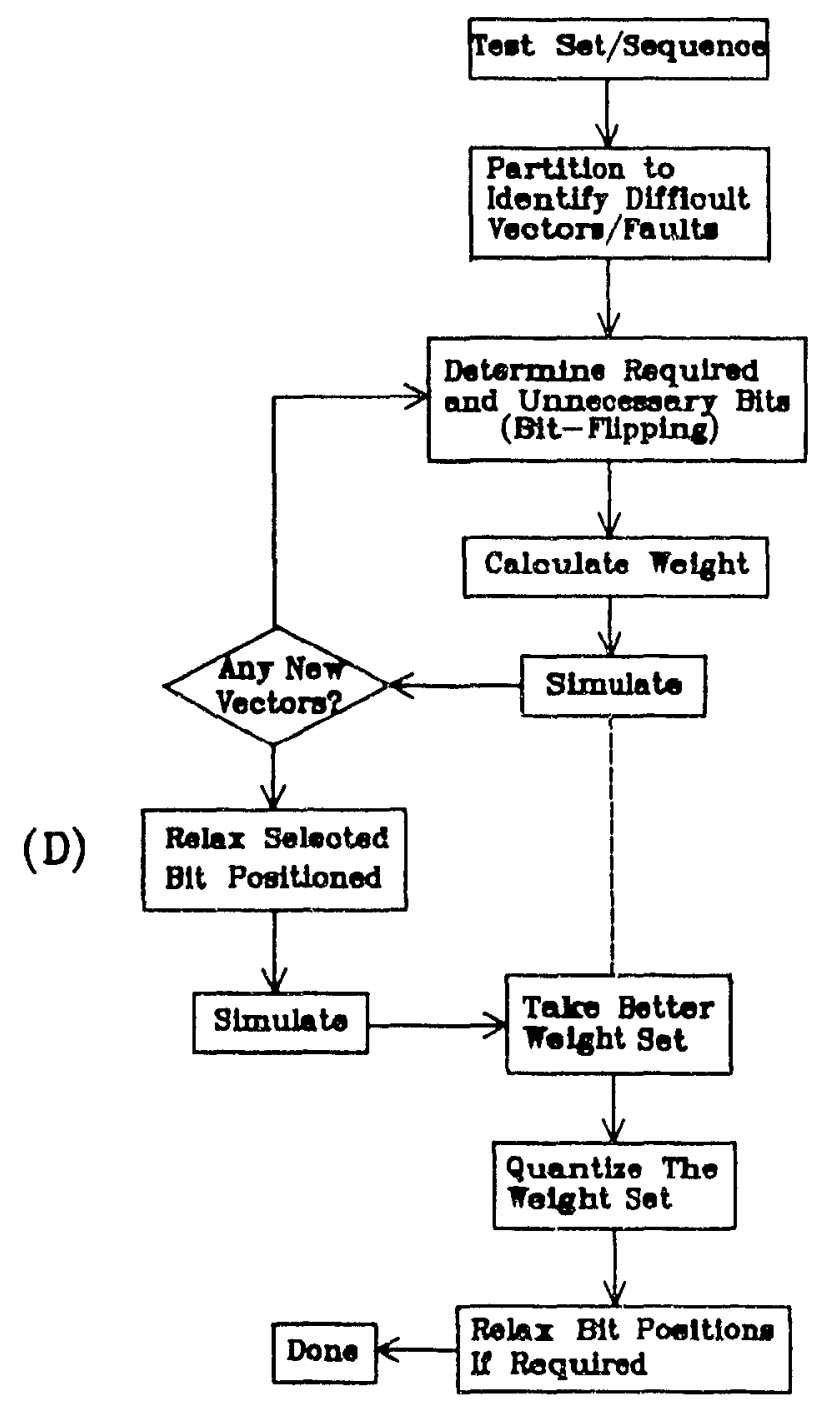

(A)

(B)

(C)

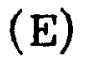

Figure 6.1 Flowchart of the Weight Estimation Procedure 
The first step (A) is to identify the required test vectors which tend to extend lhe conventional random test Jength needed to generate a lest set. This can be dome ho tracking an associated random pattern detection profile 6.2 ) and partitioning the ( urve at the point at which the rate of coverage begins to decrease by a user-defined threshold amount. Typically this might occur in the "knee" region of the curve. The groulp" of vectors found on either side of the partition point form two subsets, each of which defines a different weight.

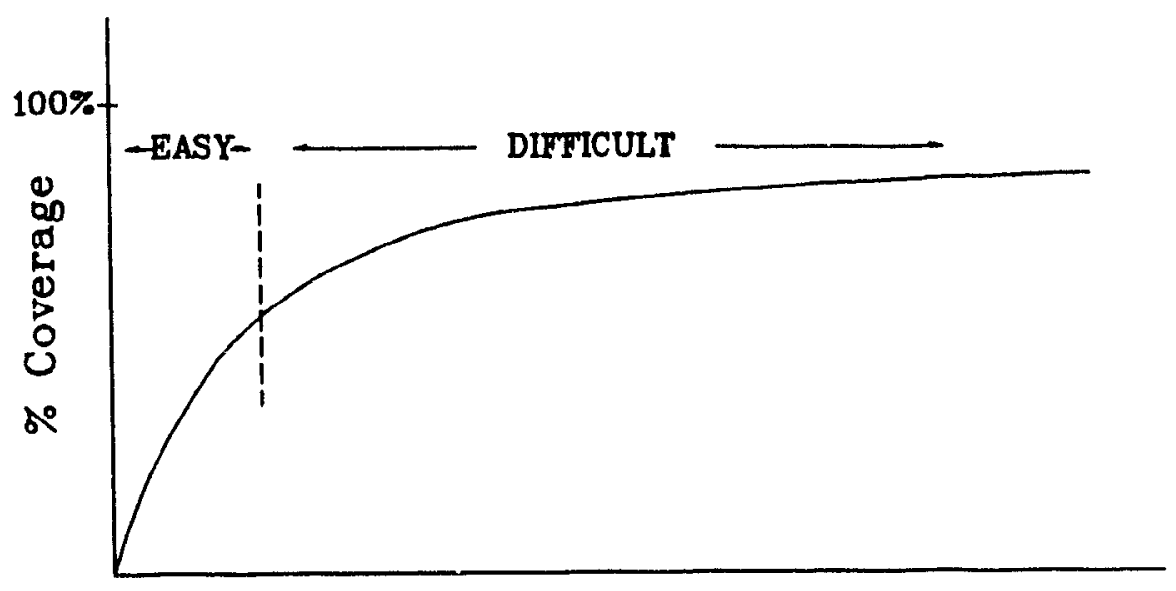

No. of Patterns

Figure 6.2 Progression of Coverage with Uniform Random Patterns

In the steeper sloped region to the left of the partition, faults classified as random easy are quickly detected in a relatively short test length. Since the perfonmance of random patterns is already acceptable in this region, a uniform weight of 0.5 (random) is uswel to cover these faults. As the test length approaches infinity, iandom difficult ${ }^{10}$ finllu are found at a comparatively slower rate. This is characterised by lhe long flattenert tail region to the right of the partition. Because the vertors orcurrmg willun thin lall

10 Note that the qualitative classification of a fault as "difficult" or "easy" is always will respect to a specifle type of generator, be it random or biased according to a specific weight set. unless specified as otherwisn "difficult" will refer to uniform I andom generation 
segment are more resistant ${ }^{11}$ to random pattern generation, a weight set is calculated to cover them (and thus their associated faults) more rapidly The experted effert of using such a pair of weights is shown in figure 6.3 .

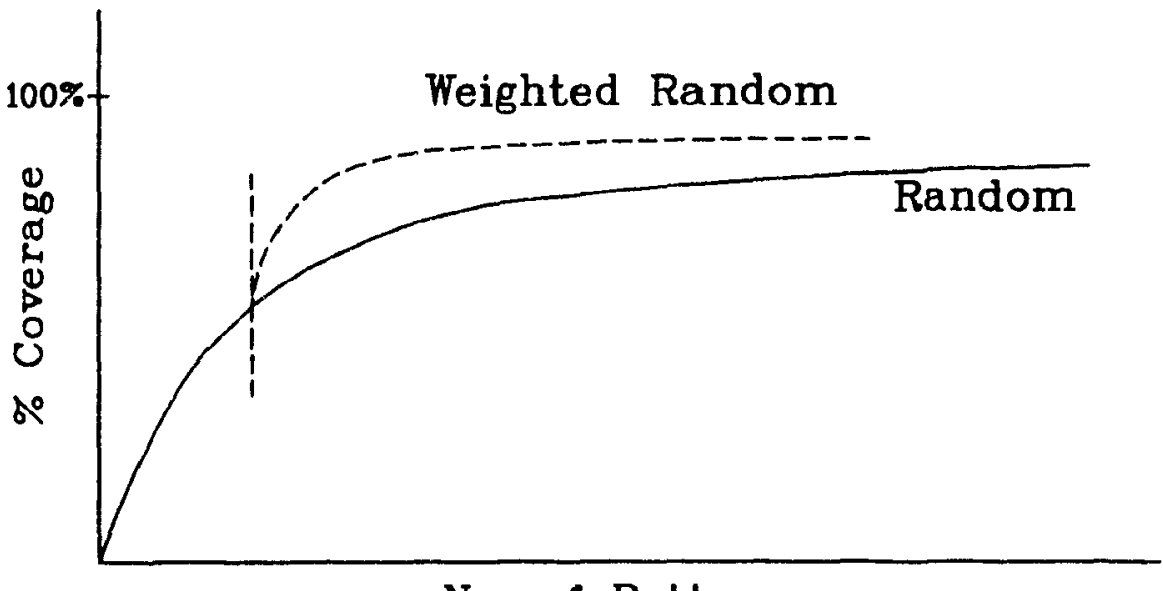

No. of Patterns

Figure 6.3 Expected effect of 2 weight sets

\subsubsection{Processing the Tail Vectors (B)}

In many cases it may be unrealistic to perform fault simulation until all difficult faults are detected. Instead, a cut-off limit may be inposed on the rate of coverage, aften which simulation is terminated In such an instance, although it is possible that not all difficult faults are covered by the test sequence, if the rate of coverage at the cut-off is chosen to be sufficiently small so that most of the difficult faults are found, the tail vectors can still be used to determine an initial non-uniform weight set. Since, aside from the circuit itself, the vectors for the detected difficult faults represent the onlv available information for determining the weight set, the estimated weight set will be geared to cover the detected difficult faults (called target laults) ATP(: tonls or a method such as that described in section 6.6 can be used to determme supplemental tail vectors for missed faults.

11 The degree of a fault's "resistance" depends on the threshold criteria. thus the position of the partition 
The pool of vectors which detect target faults is processed to provide llie necentiv information needed to calculate the non-uniform weight. Each test vector continins information concerning the circuit faults which it detects. Specifically, a subset of thr bit assignments within a vector represents a bit ordering which detects the associator faults. However, some of this information may be redundant since some fanlts are repeatedly detected by subsequent tail vectors.

In order to formulate a weight set characteristic of the target faults, an attempt is made to reduce, if not remove. any redundant information contained within each lail end vector and isolate the bit assignments useful in detecting fauls. The first task is acromplished by using a fault dropping scheme, demonstrated in figure 61 , to assign 10 each tail vector $(V)$ a list of unique target faults $(t)$ which excludes those faults deterted by any previous vector.

\begin{tabular}{|c|c|c|}
\hline Vector & Original Fault List & Assigned Fault List \\
\hline$V_{1}$ & $t_{1} t_{2} t_{3} t_{6}$ & $t_{1} t_{2} t_{3} t_{6}$ \\
\hline$V_{2}$ & $t_{1} t_{3} t_{4}$ & $t_{4}$ \\
\hline$V_{3}$ & $t_{1} t_{2} t_{3} t_{5}$ & $t_{5}$ \\
\hline
\end{tabular}

Table 6.1 Reducing the contribution of multiple detection of faults

For each tail vector, $V_{\imath}$, not all bit assignments are instrumental in detecting its associated faults. Some simulation tools [Maa88] can be used to provide a partial estimalo of the necessary bits per vector. ATPG tools can also be configured to provide some ol this information.

In the experiments performed a bit flipping algorithm was used to determine (he neederl bits per vector This is introduced here .

- Given an $n$ bit tail vector $V_{j}$, create $n$ new unique vertors $\left(r_{1}, \ldots, \nu_{2},,, v_{n}\right)$ each of whose Hamming distance from $l$; is 1 . This is done by inverting the $\imath$ th bit of $V$, to create $v_{\imath}$. 
- Simulate each vector $v_{2}$ with respect to the target faults associated wilh $V_{j}$.

- if the coverage decreases then bit $\imath$ is needed else bit $\imath$ is marked "don't care" (unnecessary)

The "filtering" process described above results in a sparse test set in which only the needed bit assignments per tail vector are retained. Using this test set representation. a weight set which can cover the set of target faults can be estimated by calculating the ratio of the number of 1 s to 0 s within each bit position (input position). A generator defined as such, attempts to produce a test sequence containing vectors similar in (ideally identical to) that of the filtered test set

\begin{tabular}{|c|cccccc|cccccc|}
\hline Vector & \multicolumn{1}{|c|}{ Before Bit } & Flipping & \multicolumn{1}{|c|}{ After Bit Flipping } \\
\hline$V_{1}$ & 1 & 1 & 1 & 0 & 0 & 1 & $\mathrm{x}$ & 1 & 1 & $\mathrm{x}$ & $\mathrm{x}$ & $\mathrm{x}$ \\
$V_{2}$ & 0 & 1 & 1 & 1 & 0 & 1 & 0 & 1 & $\mathrm{x}$ & 1 & $\mathrm{x}$ & $\mathrm{x}$ \\
$V_{3}$ & 1 & 1 & 0 & 0 & 0 & 0 & 1 & 1 & $\mathrm{x}$ & $\mathrm{x}$ & $\mathrm{x}$ & 0 \\
$V_{4}$ & 0 & 1 & 0 & 0 & 0 & 1 & 0 & 1 & 0 & $\mathrm{x}$ & $\mathrm{x}$ & $\mathrm{x}$ \\
\hline weight & .5 & 1 & .5 & .25 & 0 & .75 & .33 & 1 & .5 & 1 & .5 & 0 \\
\hline
\end{tabular}

Table 6.2 Weight Set Estimation

In table 6.2, a weight set is calculated for a sample set of vectors before and after extraction of the needed bits. Notice that that the removal of the unnecessary bits (shown as ' $x$ ' after bit flipping) results in a much different weight, for the first. fourth. fifth and sixth bit positions. Before bit flipping is performed. the definile assignments to the unnecessary bit positions act as "noise" which corrupt the weight natumate This is apparent, for example, in the sixth bit position - before bit flipping the weight is 0.75 but it is found that a 0 bias is more appropriate. Filtering off these assignments should result in a more accurite weight set. 


\subsubsection{Results I}

The test generation scheme is evaluated using five scannable circuits of varving sizn 1 ' The complexity of their topology is indicated in table 63.

\begin{tabular}{|c|c|c|c|c|c|}
\hline NETLiST & \#Faults & \# Inputs & \# Outputs & \# Gates & \# Leicls \\
\hline C6941 & 5673 & 247 & 250 & 3780 & 55 \\
\hline C9389 & 8075 & 725 & 786 & 5044 & 53 \\
\hline C11657 & 9836 & 615 & 687 & 6541 & 72 \\
\hline C30989 & 28572 & 1668 & 1668 & 16299 & 44 \\
\hline C35002 & 32985 & 1464 & 1578 & 16820 & 45 \\
\hline
\end{tabular}

Table 6.3 General Circuit Information

The first stage of the process is to determine an initial test set and ssolate the difficult faults. This is done by simulating ${ }^{13}$, with a collapsed fault set, the order of 1 million random patterns and partitioning the sequence at the point where less than roughly :30 faults were detected in a window of 3000 consecutive random patierns ${ }^{14} \mathrm{As}$ mentionsul before, the partition threshold is flexible. The results are shown in table 6.4 For earh circuit, two test lengths and their respective fault coverages in terms of the number ol irredundant faults left undetected are given. First, in column II, the test length requirer to reach the partition point (i.e. that covers the easy faults) is shown. (oolumn III list, the fault coverage of this short sequence. The maximum number of random palline simulated $\left(L_{0}\right)$ and its associated coverage are given in columms IV and $\downarrow$ resperefivels Finally, the number of target difficult faults identified in the test serpuence after 1 her

12 These were provided by Bell Northern Researcli

13 The fast fault simulator used is Tulip [Maa88] and redundancies were later found using $|\operatorname{Cox} 90|$

14 Although the initial test set was determined using simulation, it is stressed that it is not important how thr test set was developed 


\begin{tabular}{|c|c|c|c|c|c|}
\hline I & $\begin{array}{c}\text { II } \\
\text { NETLIST }\end{array}$ & $\begin{array}{c}\text { III } \\
\text { ReP. Test } \\
\text { \# Undet } \\
\text { Faults }\end{array}$ & $\begin{array}{c}\text { IV } \\
\text { Max. R.P. Test } \\
\text { Length }\left(L_{n}\right)\end{array}$ & $\begin{array}{c}\text { V } \\
\text { \# Undet } \\
\text { Fault.s }\end{array}$ & $\begin{array}{c}\text { VI } \\
\text { \# Target } \\
\text { Fault.s (V - III) }\end{array}$ \\
\hline C6941 & $55 \mathrm{~K}$ & 751 & $3 \mathrm{M}$ & 7 & 7.4 \\
\hline C9389 & $12 \mathrm{~K}$ & 508 & $02 \mathrm{M}$ & 0 & 508 \\
\hline C.11657 & $53 \mathrm{~K}$ & 517 & $1 \mathrm{M}$ & 94 & 423 \\
\hline C;30989 & $13 \mathrm{~K}$ & 1954 & $2 \mathrm{M}$ & 41 & 1913 \\
\hline C35002 & $15 \mathrm{~K}$ & 370 & $1 \mathrm{M}$ & 33 & 337 \\
\hline
\end{tabular}

Table 6.4 Initial (random pattern) Circuit Data

partition point (i.e after the test length of column II) is shown in column VI. This fault pool provides information used in the bit-flipping process.

The next step is to isolate the difficult vectors and extract, the needed hits. Using thin information, a weight set is then calculated and a set of $40 K$ WRPs is simulated (tablr 6.5). This limit on the WRP test length is chosen to satisfy approximate test time restrictions ${ }^{15}$ but in practice, of course, the upper bound can varv Column III (Lurr) of table 6.5 is the point in this WRP test sequence after which coverage of the difficult faults does not increase significantly $L_{r p}$ (col. II) is the number of extra random patterns required to cover the easy faults not yet detected. The coverage of the pair of weight sets is indicated in column IV by the number of faults left undelected after applying $L_{w r p}+L_{r p}$.

Test length improvements ranging from 1 to 3 orders of magnitude ( $i$ e powers of 10 ) are recorded. For example, more than 2 million pseudorandom patterns were needed to test C30989 with a coverage of 41 undetected faults. while 31 undetected faults remain after a joint sequence of $53 \mathrm{~K}$ patterns Also, allhough a few tanget faults are misserl. a number of previously undetected ones are found (col V) Recall that these newlv detected faults could have required a number of random patterns far in exress of the

15 Another measure of this would be to more specifically consider the scan chain length and limit the number of bits in the test sequence 


\begin{tabular}{|c|c|c|c|c|c|c|}
\hline $\begin{array}{c}\text { I } \\
\text { NETLIST }\end{array}$ & $\begin{array}{c}\text { II } \\
L_{r p}\end{array}$ & $\begin{array}{c}\text { III } \\
L_{w r p}\end{array}$ & $\begin{array}{c}\text { IV } \\
\text { \# Undet. } \\
\left(L_{w r p}+L_{r p}\right)\end{array}$ & $\begin{array}{c}\text { V } \\
\text { \# New } \\
\text { Found }\end{array}$ & $\begin{array}{c}\text { VI } \\
\text { Targets } \\
\text { Missed }\end{array}$ & $\begin{array}{c}\text { VII } \\
\text { \# Indet } \\
\left(L_{w r}+L_{t^{\prime}}\right)\end{array}$ \\
\hline C6941 & $5.5 \mathrm{~K}$ & $38 \mathrm{~K}$ & $\mathbf{8}$ & 7 & 8 & 0 \\
\hline C99389 & $12 \mathrm{~K}$ & $34 \mathrm{~K}$ & 0 & 0 & 0 & 0 \\
\hline C11657 & $5.3 \mathrm{~K}$ & $34 \mathrm{~K}$ & $\mathbf{2 4}$ & 77 & 7 & 17 \\
\hline C30989 & $13 \mathrm{~K}$ & $40 \mathrm{~K}$ & 31 & 33 & 23 & 8 \\
\hline C,35002 & $15 \mathrm{~K}$ & $37 \mathrm{~K}$ & $\mathbf{2 1}$ & 22 & 10 & 12 \\
\hline
\end{tabular}

Table 6.5 Initial WRP data

maximum amount originally simulated (table 6.4 ).

\subsection{Improving the Estimated Weight Set (C,D)}

Referring to figure 6.3 , as expected, within the WRP region there is again an extenderl tail segment where WRP difficult faults are detected. This region may be excessively long because not all faults were detected in the original random pattern simulation. and/or characteristics of all faults are not sufficiently sepresented by one non-uniform weight set.

To overcome these problems, we propose two modifications to alter the weight set. :

- Update the initial pool of target faults.

- Relax selected input biases.

These modifications are ad hoc and iterative in nature.

\subsubsection{Phase 1 - Update Target Pool (C)}

The effertiveness of the method depends on how well the existing pool of target, fault: represents all the existing difficult faults ${ }^{16}$. It is possible that simulation according In

16 In the experiments performed, redundancies were not known initially 
the the initial weight set can detect some extra faults which are not detectable using realistic random test lengths. These new test vectors are included into the target pool and the weight set is re-estimated. This process of iterative WRP generation and reestimation of the weight set repeats until no new friults are detected.

\subsubsection{Results II}

The results of Phase 1 modification of the initial weight set is shown below in table 6.6. Here the number of new faults detected (col V) does not include those found in the initial WRP run (table 6.5). 'The total number of target faults is updated to the total numbe: of difficult faults found thus far in the process.

\begin{tabular}{|c|c|c|c|c|c|c|}
\hline $\begin{array}{c}\text { I } \\
\text { NETLIST }\end{array}$ & $\begin{array}{c}\mathrm{II} \\
L_{\mathrm{rp}}\end{array}$ & $\begin{array}{c}\text { III } \\
L_{t w r p}\end{array}$ & $\begin{array}{c}\text { IV } \\
\# \text { Undet. } \\
\left(L_{u r p}+L_{r p}\right)\end{array}$ & $\begin{array}{c}\mathrm{V} \\
\text { \# New } \\
\text { Found }\end{array}$ & $\begin{array}{c}\text { VI } \\
\text { \# Target } \\
\text { Missed }\end{array}$ & $\begin{array}{c}\text { VII } \\
\# \text { Indet. } \\
\left(L_{n, r)}+L_{n}\right.\end{array}$ \\
\hline C6941 & $5.5 \mathrm{~K}$ & $27 \mathrm{~K}$ & 5 & 0 & 5 & 0 \\
\hline C9389 & $12 \mathrm{~K}$ & $34 \mathrm{~K}$ & 0 & 0 & 0 & 0 \\
\hline C11657 & $5.3 \mathrm{~K}$ & $22.2 \mathrm{~K}$ & 7 & 17 & 7 & 0 \\
\hline C30989 & $13 \mathrm{~K}$ & $40 \mathrm{~K}$ & 31 & 0 & 23 & 8 \\
\hline C.35002 & $15 \mathrm{~K}$ & $29 \mathrm{~K}$ & 12 & 4 & 5 & 7 \\
\hline
\end{tabular}

Table 6.6 Phasel runs of $40 \mathrm{~K}$ length

As expected, there is a general improvement in the weighted test length (col. III); in three cases (C6941, C11657 and C35002) accompanied by an increase in coverage The reduction of test longth may in some cases seem small but depending on the number of input nodes, the impact, on test application time can be quite significant,

\subsubsection{Phase 2 - Relax Pin Biases (D)}

In an attempt to further reduce the weighted test length, the biases of selected pins are relaxed. After a suitable weight set is found in Phase 1, the circuit is re-simulaterl 
using the weighted generator and only the target pool of faults is considered. Ignill. by noting the progression of coverage, there will be a small group of vectors which aro found after a long WRP sequence This effect is expected since one has in hemg unent to characterize many possibly different faults. The assumption is that these low WRI' difficult vectors strongly differ from the generator bias at several positions. A partial remedy is to force some of these conflicting pin biases to $50^{\text {nts }}$. This compromise ahould not adversely affect the test length if the number of positions modifierl is small

The method of selecting bit positions to be relaxed is rather straighllorwarl Accombling to a threshold criteria, vectors found in the end of the WRP tal reginn are atripper from the WRP test set. An example of such a threshold is to strip all vectors fouml after the point where no fault is detecled by 1000 consecutive WRPS

Using the algorithm of section 6.1, a weight set is estimated from the stripperl WIR vectors and is compared to the generator bias. Bit positions which dilfer by a userdefined amount ( e.g. 07 ), are relaxed. For example:

\begin{tabular}{|c|ccccccc|}
\hline Generator Weight & 1 & 0 & .2 & .7 & .8 & .2 & .1 \\
\hline Tail Weight & 1 & 0 & .4 & .8 & .1 & .9 & .3 \\
\hline New Weight & 1 & 0 & 2 & 7 & .5 & .5 & 1 \\
\hline
\end{tabular}

Note that this is a potentially volatile step because disturbing the generator hias mav negatively affect the detection of WRP easy faulis. As a result. it is recommender that this phase be performed only once and the number of positions relaxed be small.

\subsubsection{Results III}

Table 67 is the resulis after phase 2 modification.

Aside from c9389, there is little difference in the WRP test leng his of this and the phase 1 run. In 3 cases though, the coverage increased slightly. Also, notice that for C.30989, $L_{w r p}$ is the $40 \mathrm{~K}$ limit. If ten thousand more weighted patterns are simulatol, 
63 Hardware Implementation

\begin{tabular}{|c|c|c|c|c|c|c|}
\hline $\begin{array}{c}\text { I } \\
\text { NETLIST }\end{array}$ & $\begin{array}{c}\text { II } \\
L_{r p}\end{array}$ & $\begin{array}{c}\text { III } \\
L_{w r p}\end{array}$ & $\begin{array}{c}\text { IV } \\
\# \text { Undet. } \\
\left(J_{r p}+L_{w r p}\right)\end{array}$ & $\begin{array}{c}\mathrm{V} \\
\text { \# New } \\
\text { Found }\end{array}$ & $\begin{array}{c}\text { V1 } \\
\text { \# Target } \\
\text { Missed }\end{array}$ & $\begin{array}{c}\text { Vll } \\
\text { \# Bits } \\
\text { Relaxed }\end{array}$ \\
\hline C6941 & $5.5 \mathrm{~K}$ & $33 \mathrm{~K}$ & 4 & 0 & 4 & 5 \\
\hline C9389 & $12 \mathrm{~K}$ & $4 \mathrm{~K}$ & 0 & 0 & 0 & 6 \\
\hline C11657 & $53 \mathrm{~K}$ & $25 \mathrm{~K}$ & 0 & 0 & 0 & 10 \\
\hline C30989 & $13 \mathrm{~K}$ & $40 K$ & 30 & 0 & 22 & 16 \\
\hline C35002 & $15 \mathrm{~K}$ & $29 \mathrm{~K}$ & 12 & 0 & 5 & 6 \\
\hline
\end{tabular}

Table 6.7 Refıned WRP Data

the coverage is 22 undetected faults, thus illustrating the possible tradeoff between lest time and coverage. Relaxing an input's bias to 0.5 also reduces the implementaliun overhead since a modified scan cell is not needed at that position.

In all the cases, because test length did not suffer, it could be beneficial to investigate the maximum number of inputs which could be relaxed without serinusly increasing lihe WRP test length and coverage

A summary of the performance of the complete generation scheme in reducing lihe random pattern test length otherwise needed to achieve equivalent coverage, is shown in table 68 . It was mentioned earilier that weighted generation can detect faults which would require a number of random patterns far greater than the amount originally simulated $\left(L_{0}\right)$. If this trend persists in other circuits, the conventional 2 part test, generation strategy of using random vectors followed by DTPG can be modified t, include a weighted random stage after the random pattern step. The results of such a process is shown in column $\operatorname{VII}\left(L_{w r p}+L_{o}\right)$ of tables 6.5 and 66

\subsection{Hardware Implementation}

One of the major conrerns when building an on-chip generator is to minimize the aled overhead without severely compromising test quality and testability of the BIST cir- 
63 Hatdware Implementalion

\begin{tabular}{|c|c|c|c|c|c|}
\hline \multirow[b]{2}{*}{ NETLIST } & \multicolumn{3}{|c|}{ Refined WRP Data } & \multirow{2}{*}{$\begin{array}{l}\text { Equir } \\
\text { Rand Pat } \\
\text { Test Lon }\end{array}$} & \multirow{2}{*}{$\begin{array}{l}\text { lent } \\
\text { Length } \\
\text { Reduction }\end{array}$} \\
\hline & $L_{r p}$ & $L_{u, p}$ & $\begin{array}{c}\text { \# Undet } \\
\text { Faults }\end{array}$ & & \\
\hline C6941 & $5.5 \mathrm{~K}$ & $33 \mathrm{~K}$ & 4 & $4 M$ & $99^{\prime \prime} ;$ \\
\hline C9389 & $12 \mathrm{~K}$ & $4 \mathrm{~K}$ & 0 & $0.5 \mathrm{M}$ & 1720 \\
\hline C11657 & $5.3 \mathrm{~K}$ & $25 \mathrm{~K}$ & 0 & $30 \mathrm{MI}$ & (1) $)^{11}$ \\
\hline C'30989 & $13 \mathrm{~K}$ & $40 K$ & 30 & $4 \mathrm{M}$ & $\left(18^{17} ;\right.$ \\
\hline C.35002 & $15 \mathrm{~K}$ & $29 \mathrm{~K}$ & 12 & $.5 \mathrm{M}$ & $90 \%$ \\
\hline
\end{tabular}

Table 6.8 Weighted Testing $v$ s Random Testıng

cuitry. Two interdependent issues are involved in the proposed implementation stratens

- The hardware used to generate patterns accorrling to a pair of wonghi sets must be simple and transparent to the CU'T It must also be general enough to be used in any scan circuit testable with one non-unilonm weight

- The weight set determined must be adapted to suit the generator

The suggested approach is to redesign specific scan rells so that from the pattern generition point of view, the CUT appears testable with a uniformly distributed pseudorandon! sequence.

\subsubsection{Local Generation of Dually Weighted Test Patterns}

$\Lambda$ Distributed BIST Generator provides test vectors according to both the crupiprobab)/ and determined weight sets. It consists of a generatıng scan chanl (GSC:AN) and an on-chip autonomous random pattern generator such as a $C: A$ of l,FiR The glolial effect is that each gen^rator output (CUT input) can be made to be dualls weighterl - generating patterns according to the bias of the source generator. "I acording ln " hard-wired weight ( figure 6.4)

GSCAN is an altered scan chain which serves (apart from its other fumclions) as an interface between a random pattern generator and a WRP testable cirruit At a numbm 


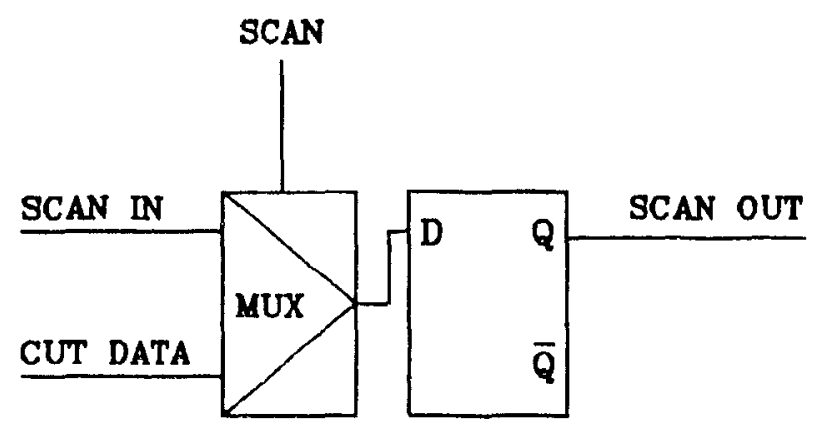

Figure 6.5 Generic Scall cell

of times proportional to the ratio of the WRP test length to the random test length. the random input at selected pin positions is locally converted to a weighted cincuil inputs of specific biases This is done by replacing standard scan elements by morlifierl blocks which perform the conversion. These new cells are themselves generic and can be included in a CAD library for zutomated design and layout.

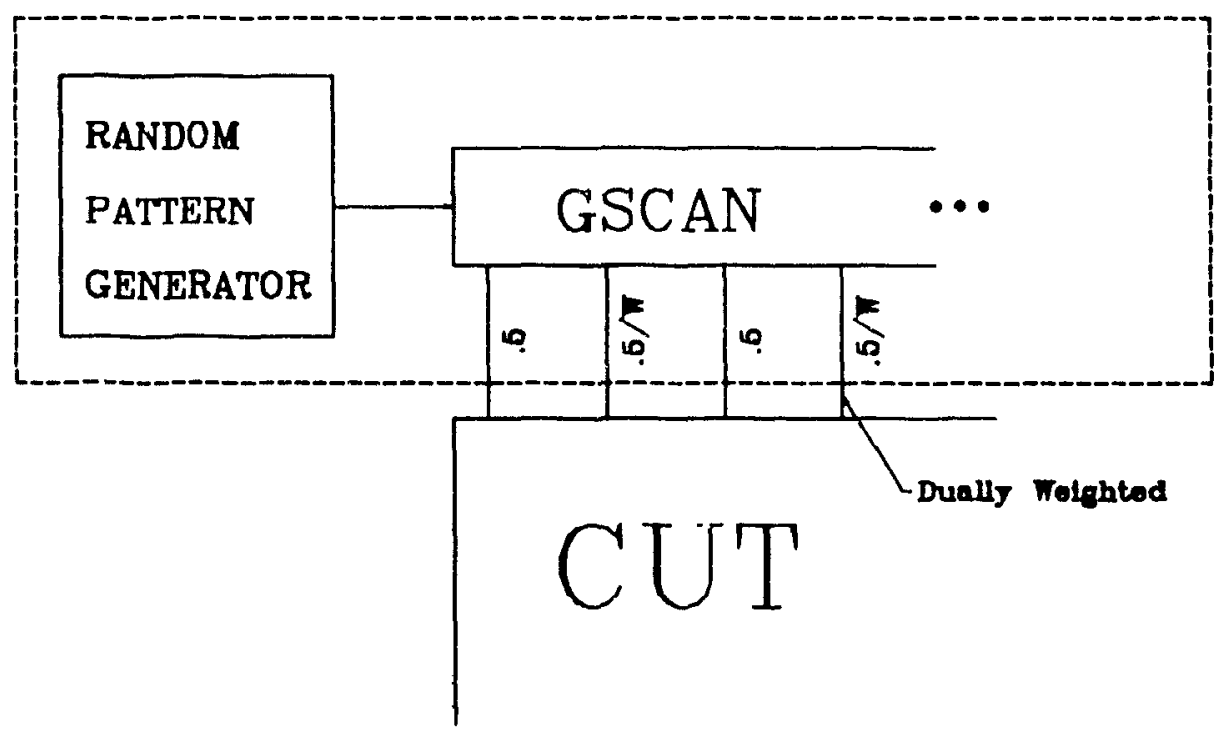

Figure 6.4 Distributed Generation of Patterns according to 2 weight scts

Since the weight set is a one to one mapping of blas values to input pins. the scan rells to be replaced are those which correspond to a bias other than 0.5 .

\subsubsection{The Circuit}




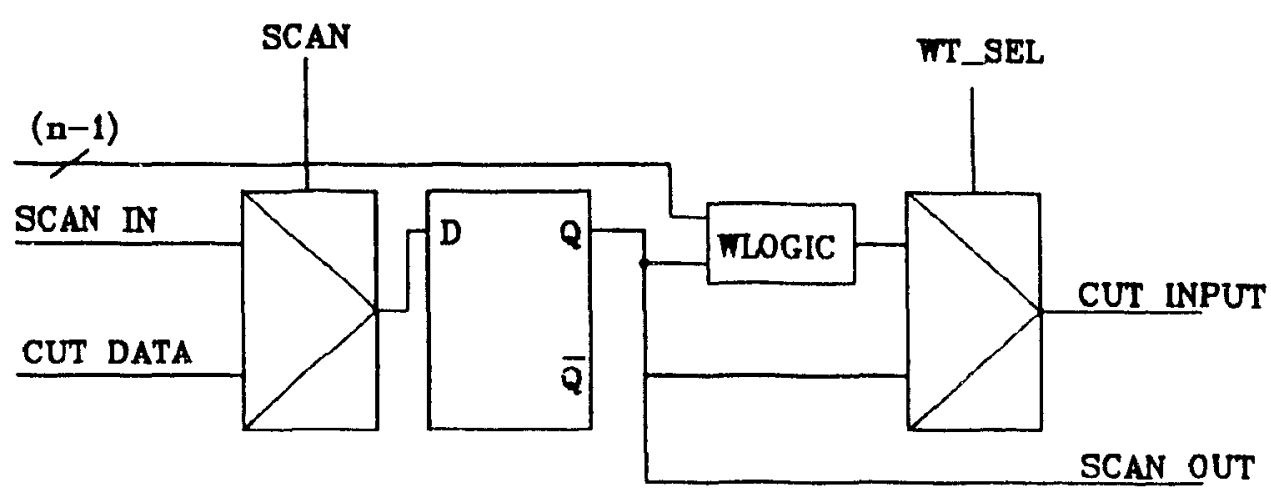

Figure 6.6 Scan cell inodified for WRP generation

The logical block diagram of the circuit for locally generating a biased input sefurenr". is shown in figure 6.6. It differs from a regular scan element (figure 6.5) in that there are :

- $n-1$ possible extra input lines from previous scan elements.

- Extra combinational logic (WLOGIC') to generate the biased stream.

- Output multiplexing to choose between biased streams

- Independence between the scan output and tie input to llie ('I'T

- An extra control line (WT-SEL) required globally

The means of generating a weighted sequence exploits the output signal probability of logic gates. Extra combinational logic (shown as WLOGIC in figure 6.6) combine"s" random input streams, one from the resident flip flop and $n-1$ from the $n-1$ previrms cells, to give a weighted output stream For example if WI,OCil: is an AND gate and $n$ is two, the bias of the weighted sequence is 0.25 .

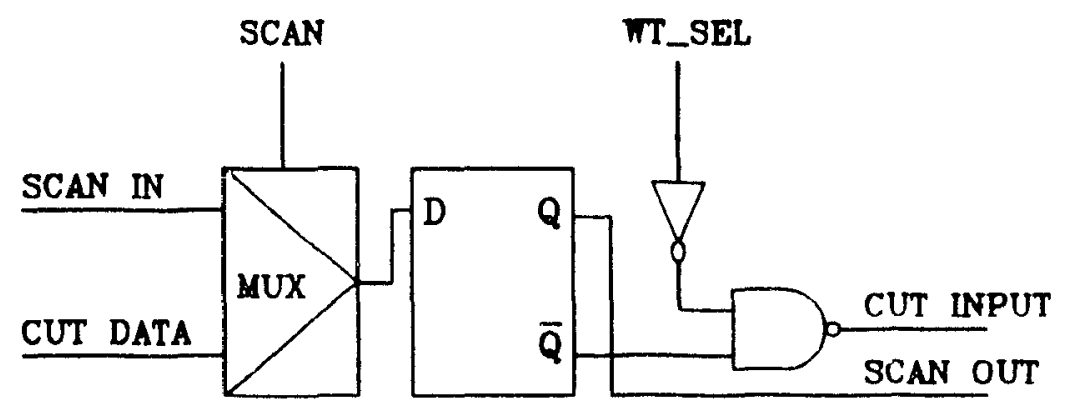

Figure 6.7 GSCAN cell for a bias of 1 
In general, the output multiplexer and WLOGIC can be represented by a small. func tionally equivalent, combinational circuit. Sample GiSCAN rell designs which ronditionally generate bit streams of bias $1,0,0.25$ and 075 are shown in figures 6.7 1.0 6.10 Mole finely tuned weights such as 0.125 and 0875 , etc., can be generated if values from greater than 1 neighbour are combined (i.e. $11 \geq 3$ ) In such cases, however, the rost of routing might become prohibitive

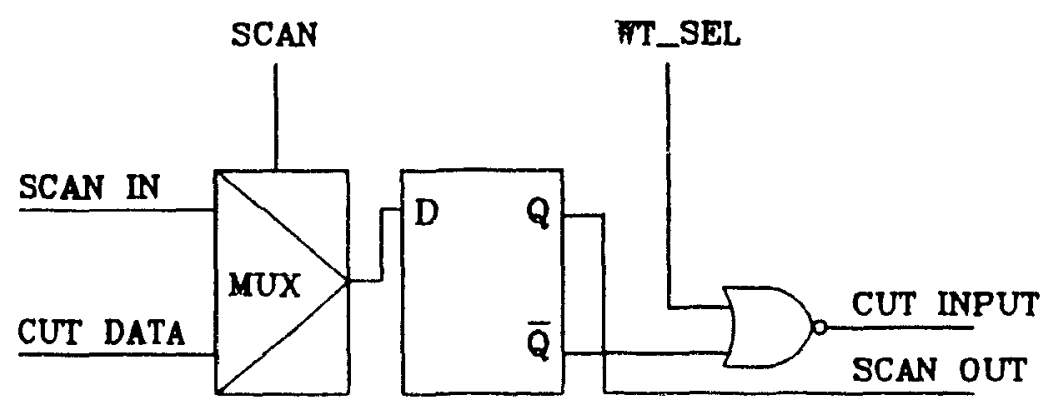

Figure 6.8 GSCAN cell for a bias of 0

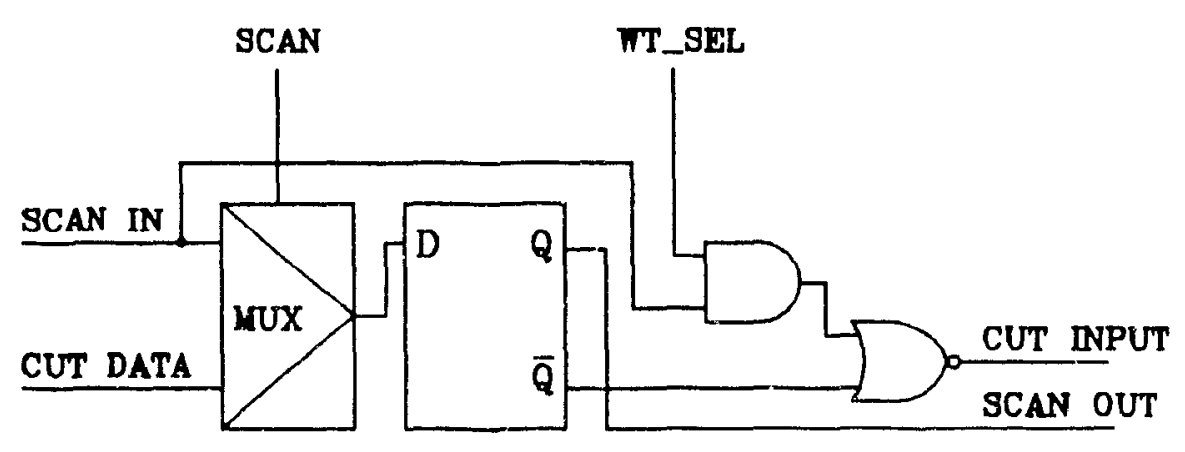

Figure 6.9 GSCAN cell for a bias of 025

\subsubsection{Mixed Generation/Application of Uniform and Weighted Patterns}

WT-SEL is a control line used to select between the weighted bit stream generaterl locally and the contents of a GSCAN cell's resident D-flip nop (D-FP). The signal ran be applied externally or, in a true BIST sense, generated by logic connected to the RPG. Furthermore, the signal can be encoded such that weighted and non-weighted test 
In general, the output multiplexer and WLOGIC call be represented by a small. linitionally equivalent, combinational curcuit Sample CiSCIAN cell designis which couch tionally generate bit streams of bias $1,0,0.25$ and 0.75 are shom generaled if values fromil More finely luned weights such as 0.125 and 0.875 . In such cases, however. the cunt il greater than 1 neighbour ale combine

routing might become prohibitive.

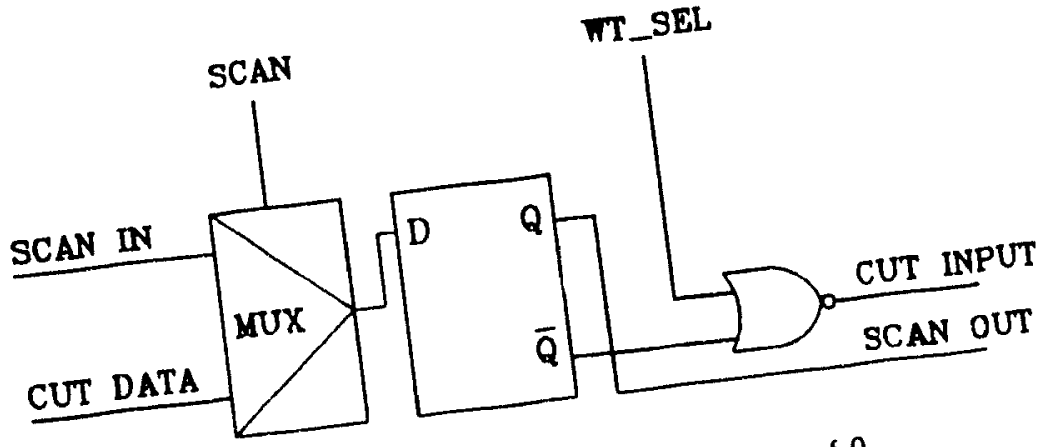

trol

Figure 6.8 GSCAN cell for a bias of 0

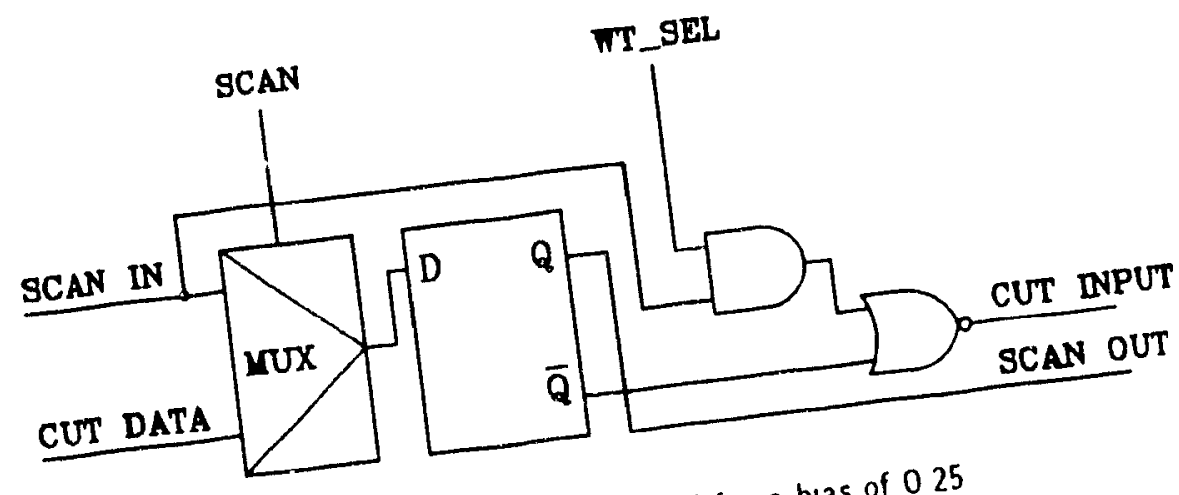

Figure 6.9 GSCAN cell for a bias of 025 WT-SEL is a control line used to select between the weighted bil strat

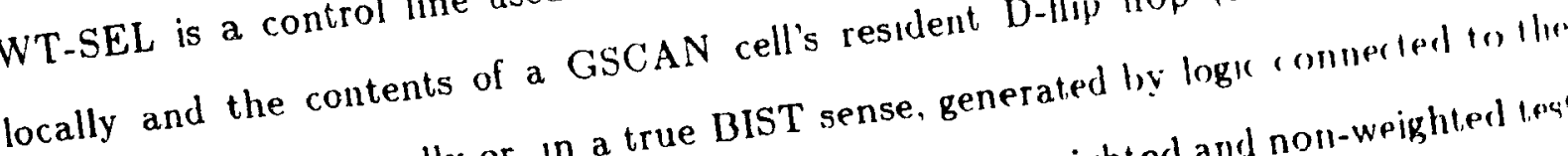
can be applied externally or, in a true BIST RPG. Furthermore, the signal can be encoded such 
During TEST, WT-SEL is itself a weighted sequence whose weight reflects the proportional sizes of the WRP and random pattern test lengths. It is used to clioose eilliel the biased stream generated or the random sequence scanned in from the RPC: This technique is possible because the order in which the individual test vertors (weighted vs. random) are applied is not critical when testing combinational circuits, using the' single stuck-at model.

\section{0 - Mode}

In order to arbitrarily select the D-FF line, the value of WT-SEL can be forced to 0 (or 1)

It is a simple task to integrate other modes of operation to satisfv other desiled sc an functions.

\subsubsection{Area Penalty}

Compared to a regular scan element, the area overhead of a redesigned scan coll in which the weighted stream is $0.25,0.75$ or 1.0 is :

- a pair of 2 input gates.

- the extra routing since the scan output is independent of the CUT input.

In the case of a 0 bias, a simple NOR ( 4 transistors) is needed. If pin biases are choscn such that inputs are needed from greater than 1 neighbour, the overhead and lavoul complexity increases due to the routing from progressively distant cells

With reference to a conventional random pattern BIS'T implementation, the extra arei penalty of this scheme is due to the contribution of each modified scan cell, the logic needed to generate WT-SEL and the additional interconnect required ton globalls rlistribute this control signal. More specific results concerning the atea overhead of the five curcuits under consideration are given in section 6.35 .1 


\subsubsection{Quantization of Pin Biases (E).}

Thresholds are ostablished to force the individual biases of the estimated weight set to implementable values. The criteria should ensure that:

- The overall features of the experimental bias, especially the extreme talues, are retained.

- The levels are chosen to facilitate minimum area overhead.

The latter point implies a tradeoff between design complexity, fault coverage and teit time. For example, the most attractive levels in terms of area and routing are $0,025,0 \%$, 0.75 and 1 . However there is a potential for loss when only these values are used ber anis extreme pin biases, such as 0.05 and 0.95 , are forced to 0.25 and 0.75 respertivels Thi'; will, in most cases, result in an increase in test length or a possible reduc tion of coverane if the test size is limited. One solution, of course, is to use more runntizalinn levele lunl this results in higher overhead.

In general, the thresholds and number of quantization levels used depend on what $k$ deemed acceptable production-wise. It will be shown experimentally in the next sertinn that biases restricted to $0,0.25,0.50 .75$ and 1 are indeed sufficient to attain accoptable fault coverage. The results of including levels 0.125 and 0.875 are also included for completeness.

\subsubsection{Results IV}

Thresholds are defined to allocate ranges of biases to fixed values The conditions land in the ensuing discussion are shown in figure 6.12.

For each test circuit the quantization effects on the weight cets found in phase 1 and phase 2 (after pin relaxing) are examined. The discussion is limited we ase 1 and rase 1 thresholds but more detailed information concerning all runs is given wh the appendix

Table 6.10 are the results of using the bias levels $-0,0.125,0.25,0.5,0.75,0.875,1$ 
CASE 1

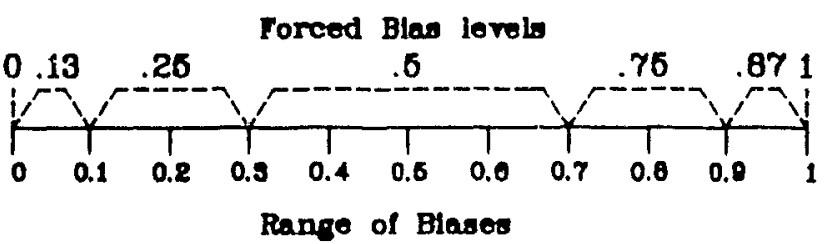

CASE 2

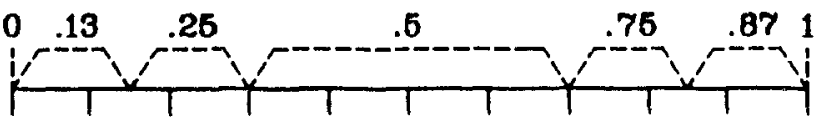

CASE 3

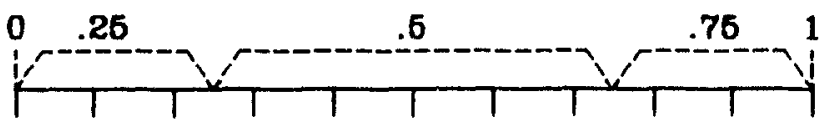

CASE 4

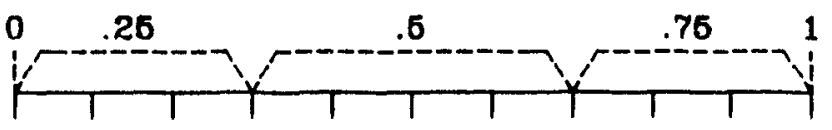

Figure 6.12 Thresholding conditions for Quantization Runs

\begin{tabular}{|c|c|c|c|c|c|c|}
\hline CASE 1 & \multicolumn{3}{|c|}{ Before Pin Relaxing } & \multicolumn{3}{c|}{ After Pin Relaxing } \\
\hline NETLIST & $L_{r p}$ & $L_{w r p}$ & $\begin{array}{c}\text { \# Undet. } \\
\text { Faults }\end{array}$ & $L_{r p}$ & $L_{w r p}$ & $\begin{array}{c}\text { \# Undet. } \\
\text { Fanlts }\end{array}$ \\
\hline C6941 & $0.5 \mathrm{~K}$ & $35 \mathrm{~K}$ & 9 & $0.5 \mathrm{~K}$ & $40 \mathrm{~K}$ & 7 \\
\hline C9389 & $11.5 \mathrm{~K}$ & $20.5 \mathrm{~K}$ & 0 & $11.5 \mathrm{~K}$ & $6 \mathrm{~K}$ & 0 \\
\hline C11657 & $5.3 \mathrm{~K}$ & $27 \mathrm{~K}$ & 9 & $5.3 \mathrm{~K}$ & $375 \mathrm{~K}$ & 4 \\
\hline C30989 & $13.5 \mathrm{~K}$ & $38.5 \mathrm{~K}$ & 56 & $13.5 \mathrm{~K}$ & $39 \mathrm{~K}$ & 54 \\
\hline C35002 & $15 \mathrm{~K}$ & $38.4 \mathrm{~K}$ & 17 & $15 \mathrm{~K}$ & $38.4 \mathrm{~K}$ & 17 \\
\hline
\end{tabular}

Table 6.10 Quantization Runs for Case 1 - 7 bias levels

and table 6.11 uses the levels $\langle 0,0.25,0.5,0.75,1\rangle$.

The impart of input pin relaxing (phase 2), in terms of covernge, is mixerl. This in itself is an interesting result When a decrease in coverage due to pin relaxing dors occur, it is very small but is accompanied by a reduction in the number of scan cells to be replaced. Thus there is possible merit to phase 2 modifications. Furthermore, it. 


\begin{tabular}{|c|c|c|c|c|c|c|}
\hline CASE 4 & \multicolumn{3}{|c|}{ Before Pin Relaxing } & \multicolumn{3}{c|}{ After Pin Relaxing } \\
\hline NETLIST & $L_{r p}$ & $L_{\text {urp }}$ & $\begin{array}{c}\text { \# Undet. } \\
\text { Faults }\end{array}$ & $L_{r r}$ & $L_{u r r}$ & $\begin{array}{c}\text { \#ndet. } \\
\text { faulls }\end{array}$ \\
\hline C6941 & $0.4 \mathrm{~K}$ & $39 \mathrm{~K}$ & 36 & $04 \mathrm{~K}$ & $39 \mathrm{~K}$ & 10 \\
\hline C9389 & $115 \mathrm{~K}$ & $205 \mathrm{~K}$ & 0 & $115 \mathrm{~K}$ & $6 \mathrm{~K}$ & 0 \\
\hline C11657 & $53 \mathrm{~K}$ & $28 \mathrm{~K}$ & 7 & $5.3 \mathrm{~K}$ & $36 \mathrm{~K}$ & 8 \\
\hline C30989 & $11.5 \mathrm{~K}$ & $39 \mathrm{~K}$ & 73 & $11.5 \mathrm{~K}$ & $395 \mathrm{~K}$ & 73 \\
\hline C35002 & $15 \mathrm{~K}$ & $308 \mathrm{~K}$ & 25 & $15 \mathrm{~K}$ & $308 \mathrm{~K}$ & 25 \\
\hline
\end{tabular}

Table 6.11 Quantization Runs for CASE 4 - 5 bias levels

is incorrect to assume that quantization performs the same degree of pin relaxing a phase 2. For example, using case 1 thresholdıng, a pin with bias 0.10 will be fored lo 0.125 whereas phase 2 operations may force it to 0.5 .

The result of using a joint scheme with 7 bias levels, in terms of both covrage and test length, is much better than that of uniform random generation alone. In a men" practical sense, the corser weight set of 5 levels uses onlv the less complex (isic:AN (all and also produces a siguificant improvement The implementation overhend (beyomil scan) for each circuit of this latter case is approximately $3 \%$ This is calculated by using an industrial standard cell library to estimate the size of each circuit and to this amount, the extra area needed in order to modify the scan chain is added. The distribution of pin biases and the size overheads cited in section 6.3.4 are used to approximate thr additional area. Information concerning input distributions and additional rums a all b" found in the appendix.

\subsubsection{LFSR-Based Scheme}

In the previous experimental analysis, software random pattenu kenerdion (therefer" almost no correlation between generated input bits) was used to evaluate lhe potent inl "1 a dually weighted generation scheme and examine the effect of using a coarse wright unt In order to evaluate the mixed-weighted implementation scheme, this section presente 
the results of simulating a model of the distributed test structure using a $32-b i t$ LF S SK (table 6.12) and 5 bias levels. The performance is compared to that of 'idcal' uniform random pattern testing implemented in software.

For cach circuit a 70 thousand mixed-weighted pattern test sequence (Test Len.) is generated with a weighted contribution indicated by the bias of the WT-SEL signal. The number of undetected faults at th? 50 thousand and 70 thousand mark is slown along with the random pattern (R P.) test length needed for equivalent roverage

\begin{tabular}{|c|c|c|c|c|}
\hline NETLIST & W'_SEL & $\begin{array}{c}\text { Mixed-Weighied } \\
\text { Test Len. }\end{array}$ & $\begin{array}{c}\text { \# Indet } \\
\text { Faults }\end{array}$ & $\begin{array}{c}\text { Equir R.P. } \\
\text { Test Len. }\end{array}$ \\
\hline C6941 & $75 \%$ & $50 \mathrm{~K}$ & 19 & $19 \mathrm{M}$ \\
& & $70 \mathrm{~K}$ & 15 & $19 \mathrm{M}$ \\
\hline C9389 & $13 \%$ & $50 \mathrm{~K}$ & 4 & $120 \mathrm{~K}$ \\
& & $70 \mathrm{~K}$ & 1 & $125 \mathrm{~K}$ \\
\hline C11657 & $75 \%$ & $50 \mathrm{~K}$ & 23 & $15 \mathrm{M}$ \\
& & $70 \mathrm{~K}$ & 13 & $-30 \mathrm{M}$ \\
\hline C30989 & $63 \%$ & $50 \mathrm{~K}$ & 95 & $1 \mathrm{M}$ \\
& & $70 \mathrm{~K}$ & 55 & $17 \mathrm{M}$ \\
& & $150 \mathrm{~K}$ & 23 & $5 \mathrm{M}$ \\
& & $200 \mathrm{~K}$ & 18 & $-5 \mathrm{M}$ \\
\hline C35002 & $37 \%$ & $50 \mathrm{~K}$ & 58 & $1.4 \mathrm{~K}$ \\
& & $70 \mathrm{~K}$ & 28 & $25 \mathrm{M}$ \\
\hline
\end{tabular}

Table 6.12 Comparison of LFSR-based Mixed-Weıghted Scheme and Random Pattern Testing

Higher fault coverage is expected if the entire analysis (partitioning fault, sets etc.) was performed using an LFSR. It has also been found from expericnce that changing the LFSR seed during test mode positively affects the rate of covelage.

Ciraphs of the progression of coverage of both the uniform random and the LFSK-baserl mixed-weighted testing techmques are given for all 5 circuits in figure 6.13 1.0 $6.17 \mathrm{~T}$ \%" test lengths are plotted on a $\log$ scale and the fault coverage is the percentage of all irredundant faults which are detected. 
63 Hardware Implementation

$x$

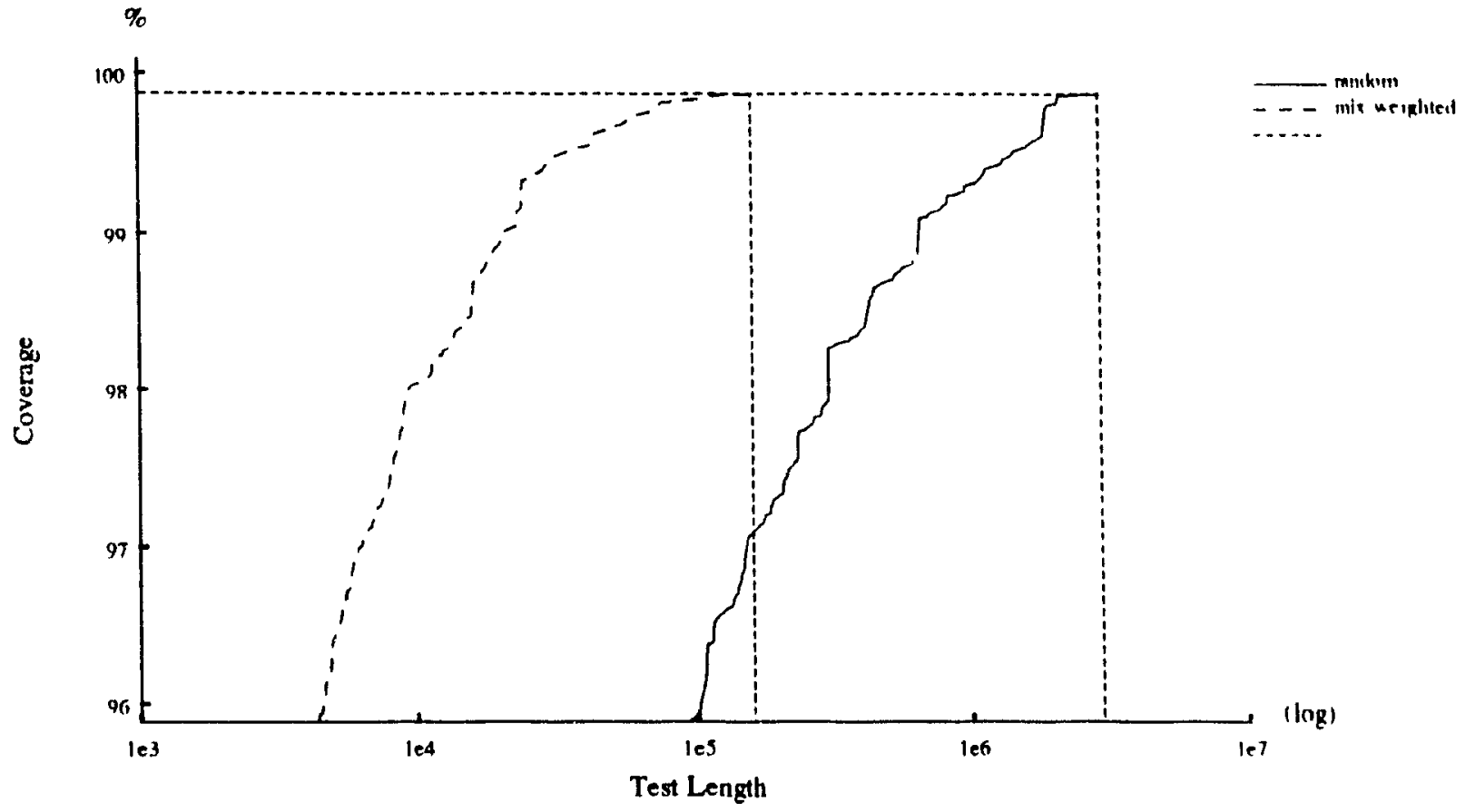

Figure 6.13 Covelage Plots for C6941

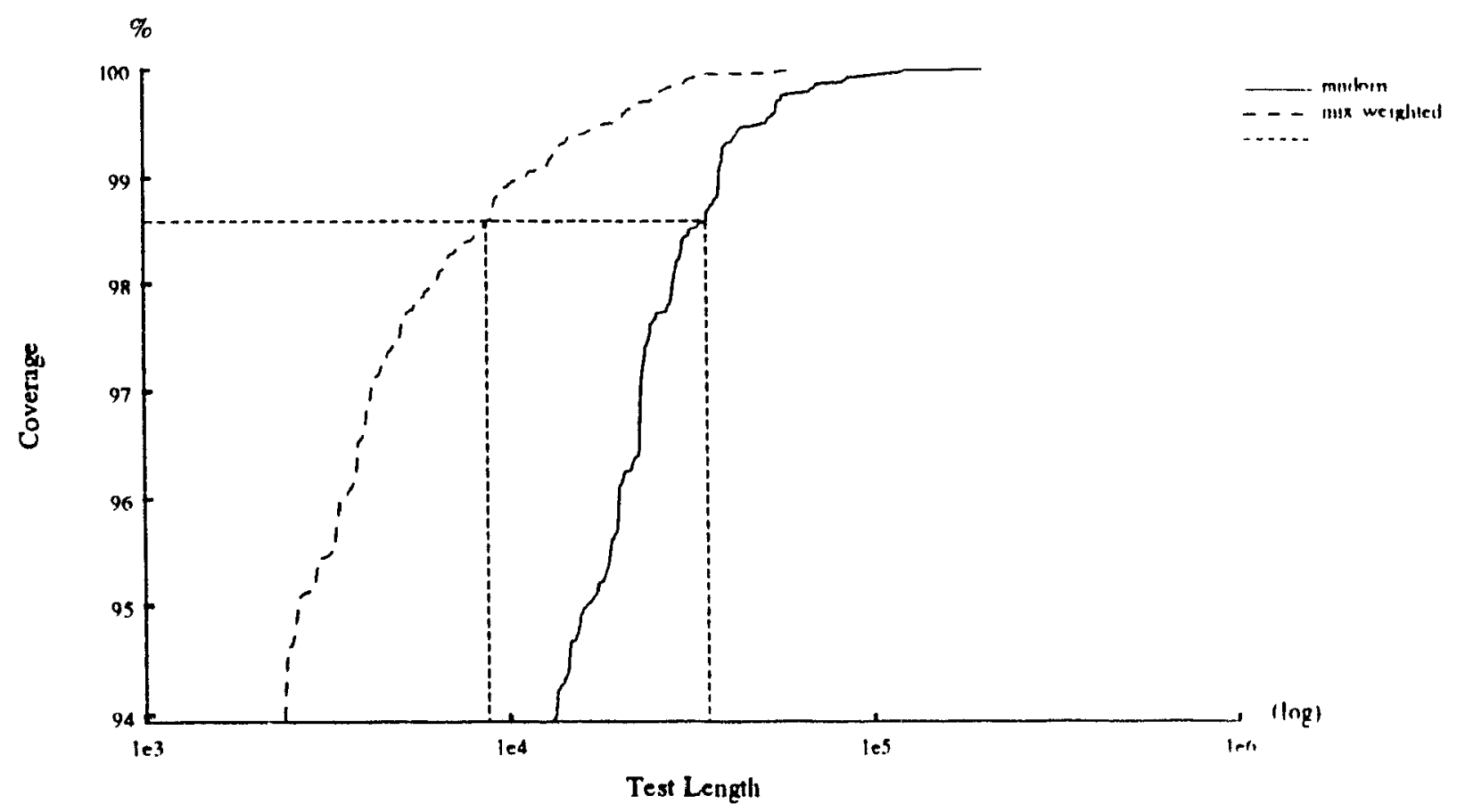

Figure 6.14 Coverage Plots for C9389 
63 Hardware Implementation

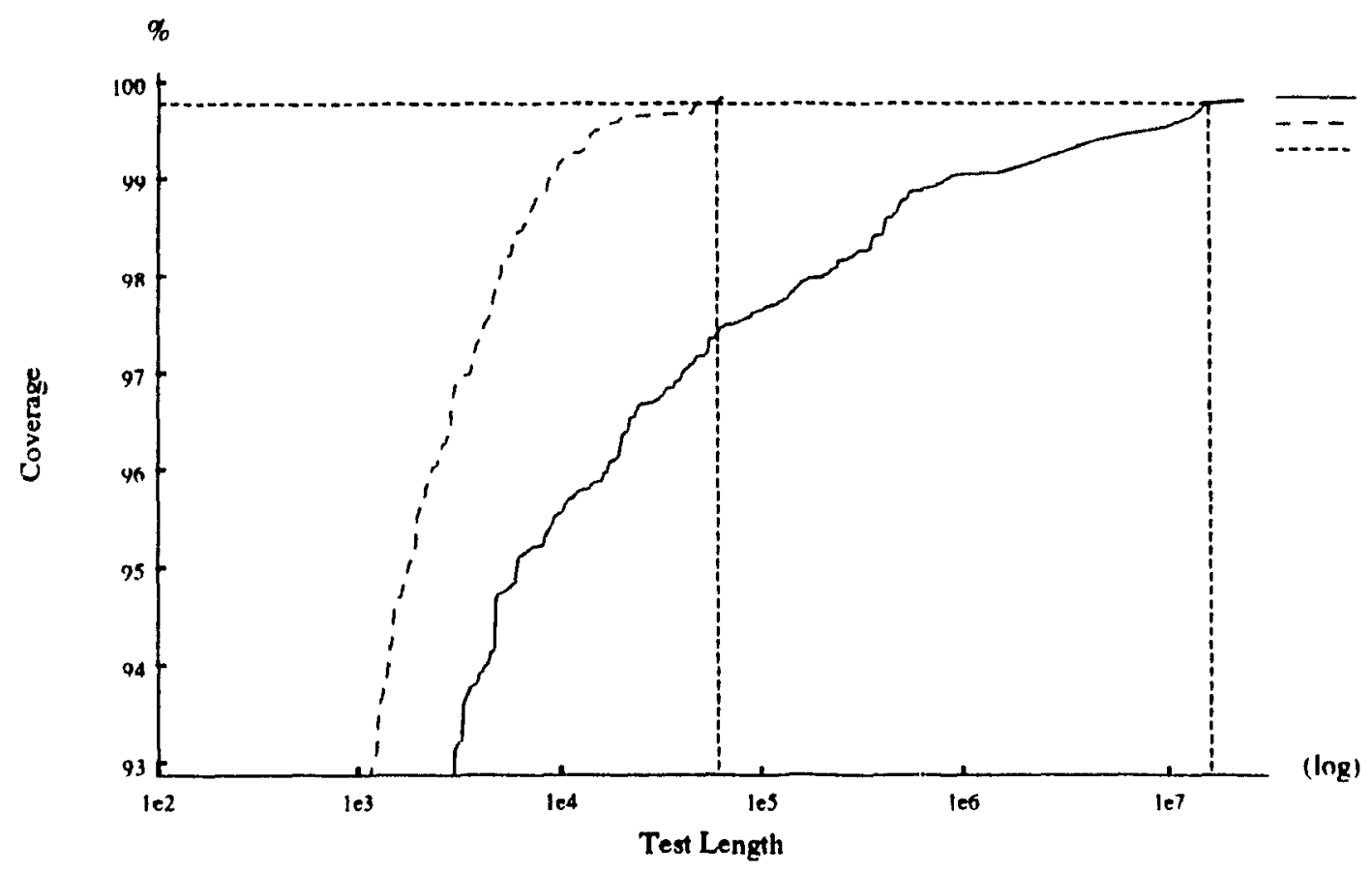

Figure 6.15 Coverage Plots for C11657

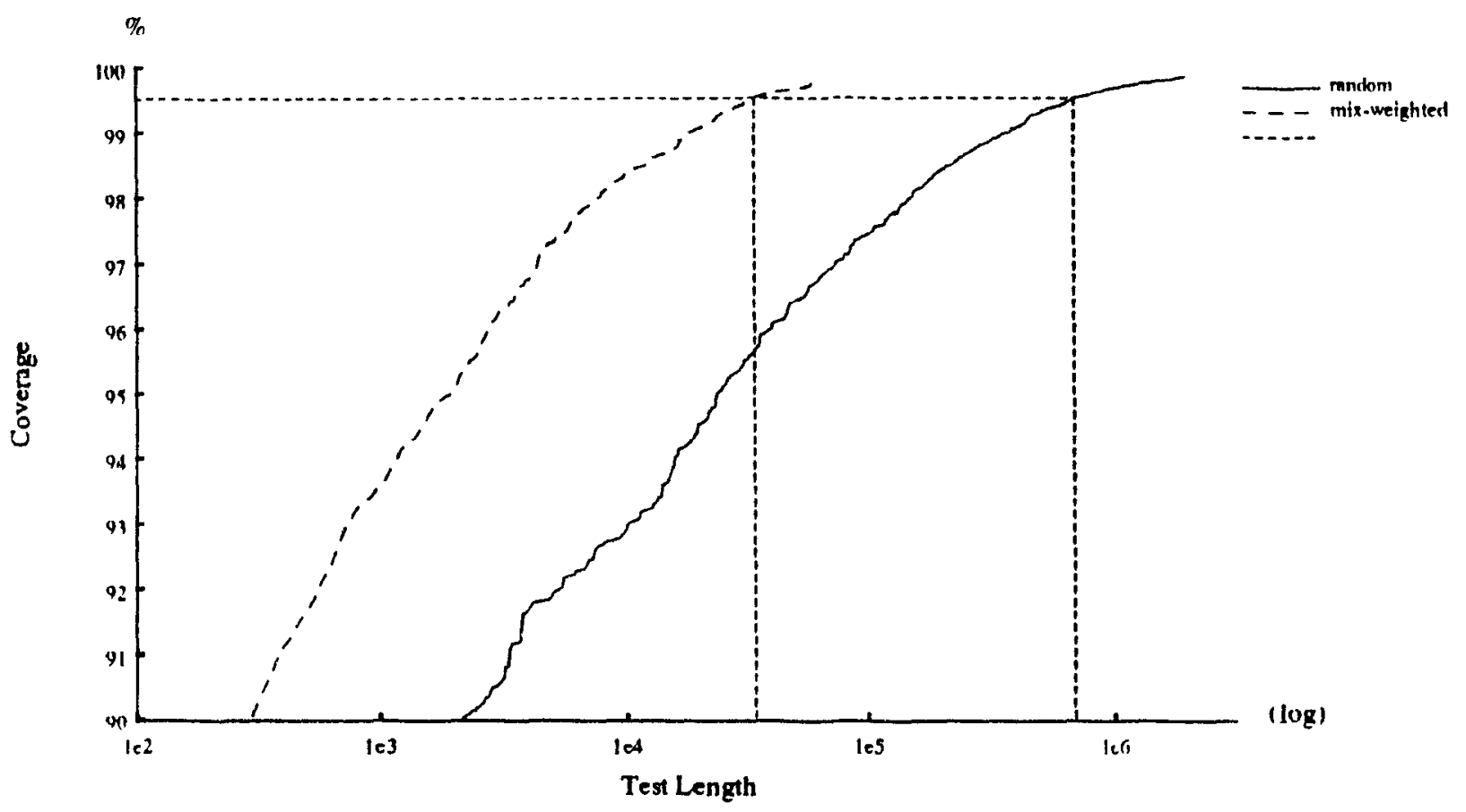

Figure 6.16 Coverage Plots for C30989 


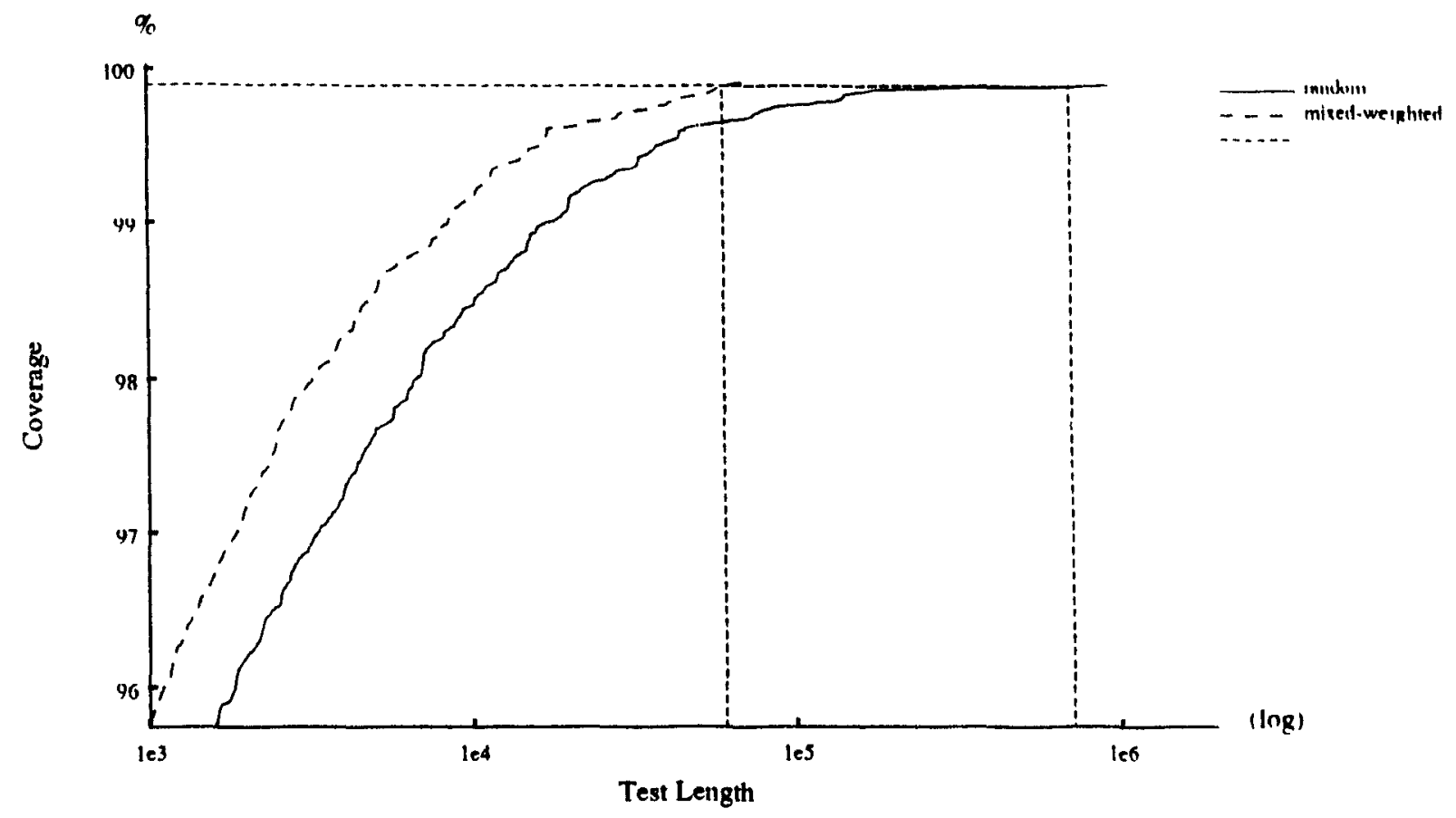

Figure 6.17 Coverage Plots for $\mathrm{C} 35002$

For these cases it is shown that random pattern test length can be reduced by orders of inagnitude using the proposed mixed-weighted generation technique.

\subsection{Other Test Circuits}

Recently, other large benchmark circuits became available [ISC89|. These sequential circuits are similar in structure to the five test circuits already considered and were adapted for experimentation by replacing flip-flop elements by input and output pins The sizes of the converted structures are indicated by the number of limes (en cillat is a combinational curcuit with 9234 lines) Experiments were performad to develop a non-quantized refined weight set. As in the previous test cases, no raim is marlo concerning the optimality of the weight however the intention is to signilicanlly remen" the random test length needed for equivalent coverage.

Table 6.13 contains some basic circuit information. Here, the total number of irrelunl- 
dant faults is in terms of a collapsed fault set, and the redundancies wete itlentifierl ${ }^{17}$ Analogous to table 6.4 , table 6.14 is the initial random pattern data used to derive thr weight sets, and in order to aid evaluation of the generation scheme the results of some extended random paltern simulations are given in table 6.15.

\begin{tabular}{|c|c|c|c|c|}
\hline NETLIST & $\begin{array}{c}\text { \#Faults } \\
\text { irredundant }\end{array}$ & $\begin{array}{c}\text { \# Faults } \\
\text { redundant }\end{array}$ & \# Inputs & \# Outputs \\
\hline C9234 & 6475 & 452 & 247 & 250 \\
\hline C13207 & 9664 & 151 & 700 & 790 \\
\hline C15850 & 11336 & 389 & 611 & 684 \\
\hline C38417 & 31015 & 165 & 1664 & 1742 \\
\hline C,38584 & 34797 & 1506 & 1464 & 1730 \\
\hline
\end{tabular}

Table 6.13 General Circuit Information

\begin{tabular}{|c|c|c|c|c|c|}
\hline $\begin{array}{c}\text { I } \\
\text { NETLIST }\end{array}$ & $\begin{array}{l}\text { II } \\
\text { R.P. Test } \\
\text { Length }\end{array}$ & $\begin{array}{l}\text { IIII } \\
\text { \# Undet } \\
\text { Faults }\end{array}$ & $\begin{array}{c}\text { IV } \\
\text { Max. R.P. Test } \\
\text { Length }\left(L_{n}\right)\end{array}$ & $\begin{array}{c}\mathrm{V} \\
\# \text { Undet } \\
\text { Faults }\end{array}$ & $\begin{array}{c}\text { VI } \\
\text { \# Target } \\
\text { Faults }(V-I I I)\end{array}$ \\
\hline C9234 & $5.5 \mathrm{~K}$ & 781 & $3 \mathrm{M}$ & 31 & 750 \\
\hline C13207 & $11.5 \mathrm{~K}$ & 589 & $0.6 \mathrm{M}$ & 0 & 589 \\
\hline C15850 & $6 \mathrm{~K}$ & 682 & $1 \mathrm{M}$ & 78 & 603 \\
\hline C:38417 & $16 \mathrm{~K}$ & 1766 & $2 \mathrm{M}$ & 32 & 1734 \\
\hline C 38584 & $15 \mathrm{~K}$ & 338 & $2 \mathrm{M}$ & 29 & 309 \\
\hline
\end{tabular}

Table 6.14 Initial (random pattern) Circuit Data

IIsing the developed refined weight set, the performance of the complete generation scheme is outlined in table 6.16. Software random pattern generation is used for this evaluation. In each case a sat of $200 \mathrm{~K}$ WRPs are simulated. Shown for each circuit is the weighted test length $\left(L_{w r p}\right)$ and corresponding fault coverage at the point at which

17 the 452 faults for 69234 are not proven redundant at this time 


\begin{tabular}{|c|c|c|}
\hline NETLIST & $L_{v^{\prime}}$ & $\begin{array}{c}\text { \# ('ndet. } \\
\text { Faults }\end{array}$ \\
\hline $\mathrm{C} 9234$ & $20 \mathrm{~N}$ & 3 \\
\hline $\mathrm{C} 13207$ & $06 \mathrm{M}$ & 0 \\
\hline $\mathrm{C} 15850$ & $120 \mathrm{M}$ & 0 \\
\hline $\mathrm{C} 38417$ & $10 \mathrm{M}$ & 5 \\
\hline $\mathrm{C} 38584$ & $10 \mathrm{M}$ & 17 \\
\hline
\end{tabular}

Table 6.15 Extended Uniform Random Pattern Circuit Data

fault detection ceased. Also, in cases where this length is in excess of $40 \mathrm{~K}$ ('(1)23.1. C38417 and C38584), the number yet undetected faults at the 40K mark is listed.

\begin{tabular}{|c|c|c|c|c|c|}
\hline \multirow{2}{*}{ NETLIST } & \multicolumn{2}{|c|}{$\begin{array}{c}\text { Refined WRP Data } \\
L_{r p}\end{array}$} & $\begin{array}{c}L_{w r p} \\
\text { \# Undet } \\
\text { Faults }\end{array}$ & $\begin{array}{c}\text { Equiv } \\
\text { Rand. Pat. } \\
\text { Test Len }\end{array}$ & $\begin{array}{c}\text { Test } \\
\text { Length } \\
\text { Redluction }\end{array}$ \\
\hline C9234 & $5.5 \mathrm{~K}$ & $40 \mathrm{~K}$ & $\mathbf{3 1}$ & $3 \mathrm{M}$ & $98^{\prime \prime}$ \\
& $55 \mathrm{~K}$ & $150 \mathrm{~K}$ & $\mathbf{0}$ & $>20 \mathrm{M}$ & $>99^{\prime \prime}$ \\
\hline $\mathrm{C} 13207$ & $11.5 \mathrm{~K}$ & $7.4 \mathrm{~K}$ & $\mathbf{0}$ & $0.6 \mathrm{M}$ & $96^{\prime \prime} \%$ \\
\hline $\mathrm{C} 15850$ & $6 \mathrm{~K}$ & $29 \mathrm{~K}$ & $\mathbf{0}$ & $120 \mathrm{M}$ & $.99^{\prime \prime} \%$ \\
\hline $\mathrm{C} 38417$ & $16 \mathrm{~K}$ & $40 \mathrm{~K}$ & 17 & $10 \mathrm{M}$ & $99^{\prime \prime}$ \\
& $16 \mathrm{~K}$ & $186 \mathrm{~K}$ & $\mathbf{0}$ & $>10 \mathrm{M}$ & $>99^{\circ} \%$ \\
\hline $\mathrm{C} 38584$ & $15 \mathrm{~K}$ & $40 \mathrm{~K}$ & $\mathbf{9}$ & $>10 \mathrm{M}$ & $>99^{\prime \prime \prime}$ \\
& $15 \mathrm{~K}$ & $77 \mathrm{~K}$ & $\mathbf{3}$ & $>10 \mathrm{M}$ & $.99^{\prime \prime} \%$ \\
\hline
\end{tabular}

Table 6.16 Weighted Testing $v s$ Random Testing

Again, it is seen that the proposed generation scheme dramatically redures the otherwine needed random test length In all cases the random pat.tern test length is redlucerl lov more than $96 \%$ for an equivalent level of coverage. 


\subsection{Comments on Overhead and Testability}

\subsubsection{Computation Overhead}

As mentioned, the thrust of this work is to provide an easily generatable test serjuencr and a corresponding BIST implementation. Defining overhead as the extra ainount of work required to realize the weighting algorithm. the effort involved in generating the initial test set is neglected since it must be coistructed anyway The overhear of the method as presented then, is due to only the bit flipping process and the extra simulation needed to verify and fine tune the determined weight sct. Other tasks such as partitioning the test sequence and calculating the weight set from processed vertors require negligible effort. In terms of the amount of patterns simula ed, the computation penalty is $O(n v)+O(m)$ simulated patterns where :

- $n=$ number of bits per vector.

- $v=$ number of vectors processed.

- $m=$ imposed maximum number of vectors in the test sequence.

- $O(m) \approx$ rough contribution of simulation needed to ver ify and morlify determined weight set ('.g. phase 1 and phase 2).

$O(n v)$ is the contribution of the bit-flipping process. The work involoved in computing the weight set from the set of processed vectors is neglegible. In the cases examined, $p$ was roughly 200 but this value varies with the chosen partition threshold between cast and difficult faults. Also, as mentioned previously, not all bits need be flipped when the vectors are processed since some simulators and DTPG tools can provide a partial estimate of the required bits.

\subsubsection{Accuracy of Extracted Data}

Even with steps taken to reduce redundancy, the data extracted "sing the bit flipping algorithm may not be optimal. This is best demonstrated by an oxample 


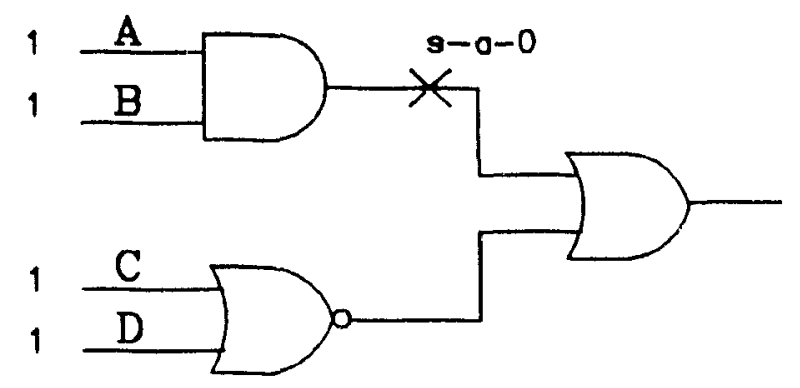

Figure 6.18 Sample Circuit for Bit Flippıng

Figure 6.18 shows a simple 4 input c11cuit with test vector '1111' used to detert th" illustrated stuck at 0 fault. Stepping through the bit flipping process (table 617 ), onls the inputs to line $A$ and $B$ are tagged as needed. The resulting proresser vertor $k$ '11XX' where ' $\mathrm{X}$ ' denotes a discarded bit.

\begin{tabular}{|c|c|c|}
\hline & $\mathrm{ABC}$ & NEEDED \\
\hline TEST VECTOR & 1111 & \\
\hline Bit 1 & $\begin{array}{lllll}0 & 1 & 1 & 1\end{array}$ & $\checkmark$ \\
\hline Bit2 & 1011 & $\checkmark$ \\
\hline Bit3 & 1101 & \\
\hline Bit4 & 1110 & \\
\hline EXTRACTED & & \\
\hline VECTOR & $11 \times x$ & \\
\hline
\end{tabular}

Table 6.17 Bit flipping for sample curcuit

By definition of a discarded bit, the specified fault should be deterted regardless of the assignments to lines $C$ and $D$. This is not true however, if $C$ and $D$ are assigned (O) ( In the test vector generated is ' $1100^{\circ}$ ). The experimental results show however, that this first order approximation of the needed bits is sufficient.

\subsubsection{Testability of Modified Scan cells}

In the implementation scheme of section 6.3 , no redundant faults are introrlurer This is demonstrated for figure 6.10 (0.75 bias) but a similar argument can be made for thr 
other modified scan cells In figure 6.19 it is assumed that line 4 (CUT INPUT) is full, testable. This is not an unreasonable assumption since this line already exists in the unmodified circuit. The problem of showing that lines $1,2,3$ and 5 are testable reduces to propagating the effec", of a fault on those lines to line 4

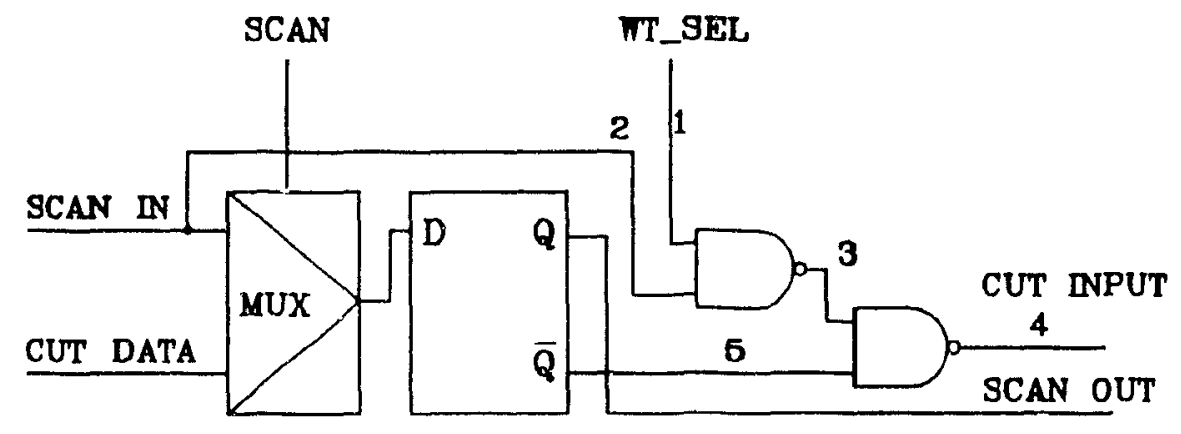

Figure 6.19 Introduced Fault Sites for a Modified Scan Cell

There are 8 possible single stuck-at faults on the target lines. Using a fault droppming scheme and recalling that line 4 is testable, four faults remain, each of which can be t.ested with the pattern(s) shown below in table 6.18 .

\begin{tabular}{|c|c|c|c|c|}
\hline Line & Fault & WT-SEL & SCAN-IN & $\bar{Q}$ \\
\hline 1 & s-a-1 & 0 & 1 & 1 \\
\hline 2 & s-a-1 & 1 & 0 & 1 \\
\hline 3 & s-a-1 & 1 & 1 & 1 \\
\hline & & 0 & 0 & 0 \\
5 & \multirow{3}{*}{ s-a-1 } & 0 & 1 & 0 \\
& & 1 & 0 & 0 \\
\hline
\end{tabular}

Table 6.18 patterns

Of course, this only shows that the introduced circuit faults are not redundant, whether or not they are artually detected depends on the test length. If they are not cleterterl within the specified test interval, a feedback loop could be added to the structure enabling the undetected fault(s) to be covered in the functional test of the scan chain.

Note that $\bar{Q}$ is used as input to the weighting logic in order to save a pair of transistors 
If this were not done, tests for stuck faults on line 5 (now mux output. Q) could lw included in the functional test of the scan chanl.

\subsubsection{Possible Missing Input State (F).}

By permuting the bits at neighbouring input sites to form weighted stimuli, it is ax. perted that some correlation between neighbouring input bits is createrl duning wrighterl pattern application.

Examples where this can occur are instances where a generic scan cell, at position 1 , is immediately followed by one modified to conditionaliy generate a bias of 0.25 or onl modified to conditionally generate a bias of 075 'The pror scenaro is Illustraterl III figure 620 .

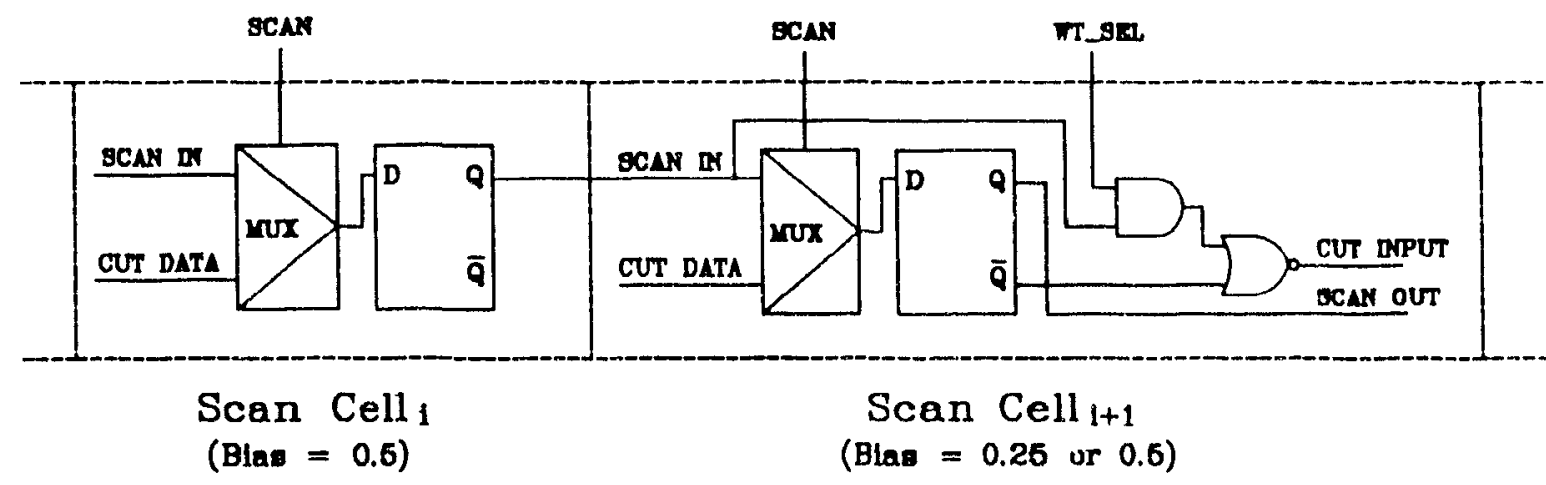

Figure 6.20 Scan Chain Ordering for Possible Missmg State

Using the GSCAN cells designed, if scan cell $\mathrm{i}+1$ conditionally generates a bras of 1$) 25$. during weighted mode the pattern ' 11 ' can never occur at posıt ions ' $1,1+1$ '. Simnilarly, Il cell $\mathrm{i}+1$ conditionally generates a bias of 0.75 , during weighted mode the pattern ' 10 ' can never occur at positions ' $i, i+1$ ' Note that the state whoch k absent reprenden lhe particular logic design used to produce the weighted out put of the risc 'IN a cll

A possible remedy to ungeneratable states is as follows By observing the lmot vere ler. for the difficult faults missed, the sites where the missing input state is critical an br. identified. If the coriesponding modified scan cell is replaced by a genoru one (I the 
bias is relaxed to 0.5 ) the problem is eliminated with little effect to the test length if 1 ,he number of positions relaxed is small. Further investigation into the impart of correlation introduced by modifying the scan chain and due to LFSR generation is terquired 


\subsection{CONCLUSIONS}

A circuit independent weighted random pattern generation scheme was ptoposed in $1 / \mathrm{lu}$ thesis. Here, a uniform random sequence and a single weighted t andom secfuence wat, shown to be highly effective in testing very large circuits containing up) fo thirtv enght thousand lines. The off-line weighting process is based on existing last fault simnlation techniques, and can be easily incorporated into most exist.ng design automatum environments (development of additional sophisticated algorithms is not enquired)

The testing scheme is geared to be suitable for a BIST application By mollaving specific scan cells, the BIST hardwate conditionally generates the weighted bit stherm locally at specific input sites This design concept can also be adapted to a globil trist generation stralegy and external testing

Apart from demonstrating that in the cases examined, one weight sot was sulficimit for a notable decrease in test length, it was also noticed that a very coarse weight ued (i.e. restricting biases to $0,0.25,0.5,0.75$ and 1) provides excellent results l'sing finer resolution within the weight set usually results in a shorter test length but at lime expense of a much ligher area penalty.

In the future, a joint scheme involving test point insertion may provide the mean of attaining $100 \%$ fault coverage in an even shorter test length. Also. it would be interstung to examine the extent in which the correlation introduced by the IIST gentidion hardware and due to LFSR generation affects the target fault coverage of such lange" circuits. 


\section{Appendix A. Extended Experimental Results}

This section contains a more detaled tabulation of results for the quantization results.s using all four thresholds of section 6.3.5.1 For each circuit, three tables are presented The first is the test lergth and coverage before and after phase 2 modification of the weight set The second and third tables are the distribution of different scan cell typec and a formal breakdown of the input biases. These tables give the absolute number of inputs which correspond to a cell type or bias. 
A Extended Experimental Result:

\begin{tabular}{|c|c|c|c|c|c|c|}
\hline C6941 & \multicolumn{2}{|c|}{ Before Pin Relaxing } & \multicolumn{3}{c|}{ After Pin Rolaxing } \\
\hline CASE & $L_{r p}$ & $L_{w r p}$ & $\begin{array}{c}\text { \# Undet. } \\
\text { Faults }\end{array}$ & $L_{r p}$ & $L_{u r p}$ & $\begin{array}{c}\text { \# Inclet } \\
\text { Faults }\end{array}$ \\
\hline 1 & $0.5 \mathrm{~K}$ & $35 \mathrm{~K}$ & 9 & $0.5 \mathrm{~K}$ & $40 \mathrm{~K}$ & 7 \\
\hline 2 & $0.5 \mathrm{~K}$ & $40 \mathrm{~K}$ & 8 & $0.5 \mathrm{~K}$ & $40 \mathrm{~K}$ & 8 \\
\hline 3 & $1.8 \mathrm{~K}$ & $39 \mathrm{~K}$ & 38 & $1.8 \mathrm{~K}$ & $39 \mathrm{~K}$ & 42 \\
\hline 4 & $0.4 \mathrm{~K}$ & $39 \mathrm{~K}$ & 36 & $0.4 \mathrm{~K}$ & $39 \mathrm{~K}$ & 40 \\
\hline
\end{tabular}

Table A.1 Quantization Runs for C6941

\begin{tabular}{|c|c|c|c|c|c|c|c|c|c|c|c|c|c|c|}
\hline C6941 & \multicolumn{10}{|c|}{ Number of Pins Biased to Specified Levels } \\
\hline & \multicolumn{1}{|c|}{ 'hase 1} \\
\hline CASE & 0 & .13 & .25 & .50 & .75 & .87 & 1 & 0 & .13 & .25 & .50 & 75 & .87 & 1 \\
\hline 1 & 34 & 10 & 19 & 126 & 24 & 28 & 6 & 33 & 10 & 17 & 131 & 21 & 26 & 6 \\
\hline 2 & 34 & 13 & 16 & 126 & 32 & 20 & 6 & 33 & 12 & 15 & 131 & 30 & 20 & 6 \\
\hline 3 & 34 & - & 17 & 149 & 41 & - & 6 & 33 & - & 15 & 154 & 39 & - & 6 \\
\hline 4 & 34 & - & 29 & 129 & 49 & - & 6 & 33 & - & 27 & 134 & 47 & - & 6 \\
\hline
\end{tabular}

Table A.2 Pin Bias Distribution for C6941 


\begin{tabular}{|c|c|c|c|c|c|c|}
\hline C9389 & \multicolumn{2}{|c|}{ Before Pin Relaxing } & \multicolumn{3}{c|}{ After Pin Prlaxing } \\
\hline CASE & $L_{r p}$ & $L_{w r p}$ & $\begin{array}{c}\text { \# Undet } \\
\text { Faults }\end{array}$ & $L_{r p}$ & $L_{w r p}$ & $\begin{array}{c}\text { \# Undet } \\
\text { Faults }\end{array}$ \\
\hline 1 & $11.5 \mathrm{~K}$ & $20.5 \mathrm{~K}$ & 0 & $11.5 \mathrm{~K}$ & $6 \mathrm{~K}$ & 0 \\
\hline 2 & $11.5 \mathrm{~K}$ & $205 \mathrm{~K}$ & 0 & $11.5 \mathrm{~K}$ & $6 \mathrm{~K}$ & 0 \\
\hline 3 & $11.5 \mathrm{~K}$ & $205 \mathrm{~K}$ & 0 & $11.5 \mathrm{~K}$ & $6 \mathrm{~K}$ & 0 \\
\hline 4 & $11.5 \mathrm{~K}$ & $20.5 \mathrm{~K}$ & 0 & $11.5 \mathrm{~K}$ & $6 \mathrm{~K}$ & 0 \\
\hline
\end{tabular}

Table A.3 Quantization Runs for C9389

\begin{tabular}{|c|c|c|c|c|c|c|c|c|c|c|c|c|c|c|}
\hline C9389 & \multicolumn{10}{|c|}{ Number of Pins Biased to Specified Levels } \\
\hline & \multicolumn{10}{|c|}{1} \\
\hline C.ASE & 0 & .13 & .25 & .50 & .75 & .87 & 1 & 0 & .13 & .25 & .50 & .75 & 87 & 1 \\
\hline 1 & 227 & - & 40 & 318 & 31 & 19 & 90 & 227 & - & 40 & 318 & 31 & 19 & 90 \\
\hline 2 & 227 & 4 & 36 & 318 & 45 & 5 & 90 & 227 & 4 & 36 & 318 & 15 & 5 & 90 \\
\hline 3 & 227 & - & 37 & 328 & 42 & - & 91 & 227 & - & 33 & 333 & 42 & - & 90 \\
\hline 4 & 227 & - & 45 & 314 & 48 & - & 91 & 227 & - & 40 & 320 & 48 & - & 90 \\
\hline
\end{tabular}

Table A.4 Pin Bias Distribution for C9389 
A Extended Experimental Rosulic

\begin{tabular}{|c|c|c|c|c|c|c|}
\hline C11657 & \multicolumn{2}{|c|}{ Before Pin Relaxing } & \multicolumn{3}{c|}{ Alter Pin Relaxing } \\
\hline CASE & $L_{r p}$ & $L_{w r p}$ & $\begin{array}{c}\text { \# Undel. } \\
\text { Faulls }\end{array}$ & $L_{r p^{\prime}}$ & $L_{u^{\prime} p}$ & $\begin{array}{c}\text { \# Indel. } \\
\text { Faults }\end{array}$ \\
\hline 1 & $5.3 \mathrm{~K}$ & $27 \mathrm{~K}$ & 9 & $53 \mathrm{~K}$ & $37.5 \mathrm{~K}$ & 4 \\
\hline 2 & $5.3 \mathrm{~K}$ & $27 \mathrm{~K}$ & 8 & $53 \mathrm{~K}$ & $375 \mathrm{~K}$ & 1 \\
\hline 3 & $5.3 \mathrm{~K}$ & $375 \mathrm{~K}$ & 18 & $53 \mathrm{~K}$ & $375 \mathrm{~K}$ & 20 \\
\hline 4 & $5.3 \mathrm{~K}$ & $28 \mathrm{~K}$ & 7 & $5.3 \mathrm{~K}$ & $36 \mathrm{~K}$ & 8 \\
\hline
\end{tabular}

Table A.5 Quantization Runs for C11657

\begin{tabular}{|c|c|c|c|c|c|c|c|c|c|c|c|c|c|c|}
\hline C11657 & \multicolumn{10}{|c|}{ Pumber of Pins Biased to Specified Levels } \\
\hline & \multicolumn{10}{|c|}{1} \\
\hline CASE & 0 & .13 & .25 & .50 & .75 & .87 & 1 & 0 & 13 & .25 & .50 & .75 & .87 & 1 \\
\hline 1 & 100 & 36 & 106 & 286 & 17 & 36 & 34 & 100 & 36 & 99 & 296 & 17 & 33 & 3.3 \\
\hline 2 & 100 & 56 & 86 & 286 & 27 & 26 & 34 & 100 & 55 & 80 & 296 & 25 & 25 & 34 \\
\hline 3 & 100 & - & 106 & 335 & 40 & - & 34 & 100 & - & 102 & .342 & 37 & - & 3.31 \\
\hline 4 & 100 & - & 142 & 286 & 53 & - & 34 & 100 & - & 135 & 296 & 50 & - & 34 \\
\hline
\end{tabular}

Table A.6 Pin Bias Distribution for C11657 
A Extended Experimental Results

\begin{tabular}{|c|c|c|c|c|c|c|}
\hline C30989 & \multicolumn{3}{|c|}{ Before Pin Relaxing } & \multicolumn{3}{c|}{ After Pin Relaxing } \\
\hline CASE & $L_{r p}$ & $L_{w r p}$ & $\begin{array}{c}\text { \# Undet. } \\
\text { Faults }\end{array}$ & $L_{r p}$ & $L_{w r} r$ & $\begin{array}{c}\text { \# Undet. } \\
\text { Faults }\end{array}$ \\
\hline 1 & $13.5 \mathrm{~K}$ & $38.5 \mathrm{~K}$ & 56 & $13.5 \mathrm{~K}$ & $39 \mathrm{~K}$ & 54 \\
\hline 2 & $13.5 \mathrm{~K}$ & $38.5 \mathrm{~K}$ & 58 & $135 \mathrm{~K}$ & $39 \mathrm{~K}$ & 5.5 \\
\hline 3 & $13 \mathrm{~K}$ & $39.5 \mathrm{~K}$ & 108 & $12 \mathrm{~K}$ & $39.5 \mathrm{~K}$ & 121 \\
\hline 4 & $11.5 \mathrm{~K}$ & $39 \mathrm{~K}$ & 73 & $11.5 \mathrm{~K}$ & $395 \mathrm{~K}$ & 73 \\
\hline
\end{tabular}

Table A.7 Quantization Runs for C30989

\begin{tabular}{|c|c|c|c|c|c|c|c|c|c|c|c|c|c|c|}
\hline C30989 & \multicolumn{10}{|c|}{ Number of Pins Biased to Specified Levels } \\
\hline & \multicolumn{10}{|c|}{ Phase 1} \\
\hline CASE & 0 & .13 & .25 & .50 & .75 & .87 & 1 & 0 & .13 & .25 & .50 & .75 & .87 & 1 \\
\hline 1 & 261 & 20 & 114 & 1117 & 75 & 47 & 34 & 261 & 20 & 108 & 1134 & 70 & 41 & 34 \\
\hline 2 & 261 & 39 & 95 & 1117 & 95 & 27 & 34 & 261 & 38 & 90 & 1134 & 85 & 26 & 31 \\
\hline 3 & 261 & - & 85 & 1202 & 86 & - & 34 & 261 & - & 77 & 1218 & 78 & - & .34 \\
\hline 4 & 261 & - & 134 & 1124 & 115 & - & 34 & 261 & - & 128 & 1137 & 108 & - & 34 \\
\hline
\end{tabular}

Table A.8 Pin Bias Distribution for C30989 
A Extended Expenmental Rerinles

\begin{tabular}{|c|c|c|c|c|c|c|}
\hline C35002 & \multicolumn{2}{|c|}{ Before Pin Relaxing } & \multicolumn{3}{|c|}{ After Pin Relaxilg } \\
\hline CASE & $L_{r p}$ & $L_{w, p}$ & $\begin{array}{c}\text { \# Undet. } \\
\text { Faults }\end{array}$ & $L_{r} r$ & $L_{u \prime \prime} r$ & $\begin{array}{c}\text { \# Indet. } \\
\text { Faults }\end{array}$ \\
\hline 1 & $15 \mathrm{~K}$ & $38.4 \mathrm{~K}$ & 17 & $15 \mathrm{~K}$ & $38.4 \mathrm{~K}$ & 17 \\
\hline 2 & $15 \mathrm{~K}$ & $30.8 \mathrm{~K}$ & 25 & $15 \mathrm{~K}$ & $308 \mathrm{~K}$ & 25 \\
\hline 3 & $15 \mathrm{~K}$ & $30.8 \mathrm{~K}$ & 30 & $15 \mathrm{~K}$ & $30.8 \mathrm{~K}$ & 30 \\
\hline 4 & $15 \mathrm{~K}$ & $308 \mathrm{~K}$ & 25 & $15 \mathrm{~K}$ & $30.8 \mathrm{~K}$ & 25 \\
\hline
\end{tabular}

Table A.9 Quantization Runs for C35002

\begin{tabular}{|c|c|c|c|c|c|c|c|c|c|c|c|c|c|c|}
\hline C35002 & \multicolumn{10}{|c|}{ Number of Pins Biased to Specified Levels } \\
\hline & \multicolumn{10}{|c|}{ Phase 1} & \multicolumn{1}{|c|}{ 'hase 2} \\
\hline CASE & 0 & .13 & .25 & .50 & .75 & 87 & 1 & 0 & .13 & .25 & .50 & .75 & .87 & 1 \\
\hline 1 & 422 & 11 & 41 & 834 & 19 & 5 & 132 & 422 & 11 & 40 & 837 & 19 & 5 & 130 \\
\hline 2 & 422 & 19 & 33 & 834 & 21 & 3 & 132 & 422 & 19 & 32 & 837 & 21 & 3 & 130 \\
\hline 3 & 422 & - & 51 & 844 & 15 & - & 132 & 422 & - & 50 & 847 & 15 & - & 130 \\
\hline 4 & 422 & - & 52 & 835 & 23 & - & 132 & 422 & - & 51 & 836 & 23 & - & 130 \\
\hline
\end{tabular}

Table A.10 Pin Bias Distribution for C 35002 
A Extended Experimental Resulta

The results of simulating the modeled mixed-weighted scheme with varving hiasm an! the WT-SEL control line, are presented in table A 11 The coverages at $50 \mathrm{~K}$ and 7 (1)k are given.

\begin{tabular}{|c|c|c|c|}
\hline NETLIST & W_SEL & \#Undet $50 k$ & \#Undet $70 K$ \\
\hline \multirow[t]{4}{*}{ C6941 } & $75 \%$ & 19 & 15 \\
\hline & 87 & 27 & 17 \\
\hline & 94 & 26 & 19 \\
\hline & 97 & 32 & 29 \\
\hline \multirow[t]{5}{*}{ C9389 } & 6 & 5 & 1 \\
\hline & 9 & 4 & 1 \\
\hline & 13 & 4 & 1 \\
\hline & 25 & 10 & 7 \\
\hline & 50 & 13 & 10 \\
\hline \multirow[t]{4}{*}{ C11657 } & 63 & 23 & 14 \\
\hline & 70 & 25 & 15 \\
\hline & 75 & 23 & 15 \\
\hline & 85 & 31 & 23 \\
\hline \multirow[t]{4}{*}{ C30989 } & 50 & 101 & 57 \\
\hline & 63 & 95 & 55 \\
\hline & 70 & 101 & 62 \\
\hline & 75 & 95 & 71 \\
\hline \multirow[t]{5}{*}{ C35002 } & 25 & 62 & 48 \\
\hline & 37 & 58 & 28 \\
\hline & 50 & 71 & 36 \\
\hline & 63 & 68 & 41 \\
\hline & 70 & 92 & 51 \\
\hline
\end{tabular}

Table A.11 Extended Results for the modeled BIST Implementation witl 32 bit LFSR

Figures A.1 through A.5 compare the test application time for the mixerl-weightherl simulation and the uniform random pattern testing. Application time and test lengt/s are linearly related, however these figures are given since the log scales used when discussing test lengths may tend to obscure the magnilude of the actual time saverl. $A$ test frequency of $2 \mathrm{MHz}$ is used. 
A Extended Expcrimental Results

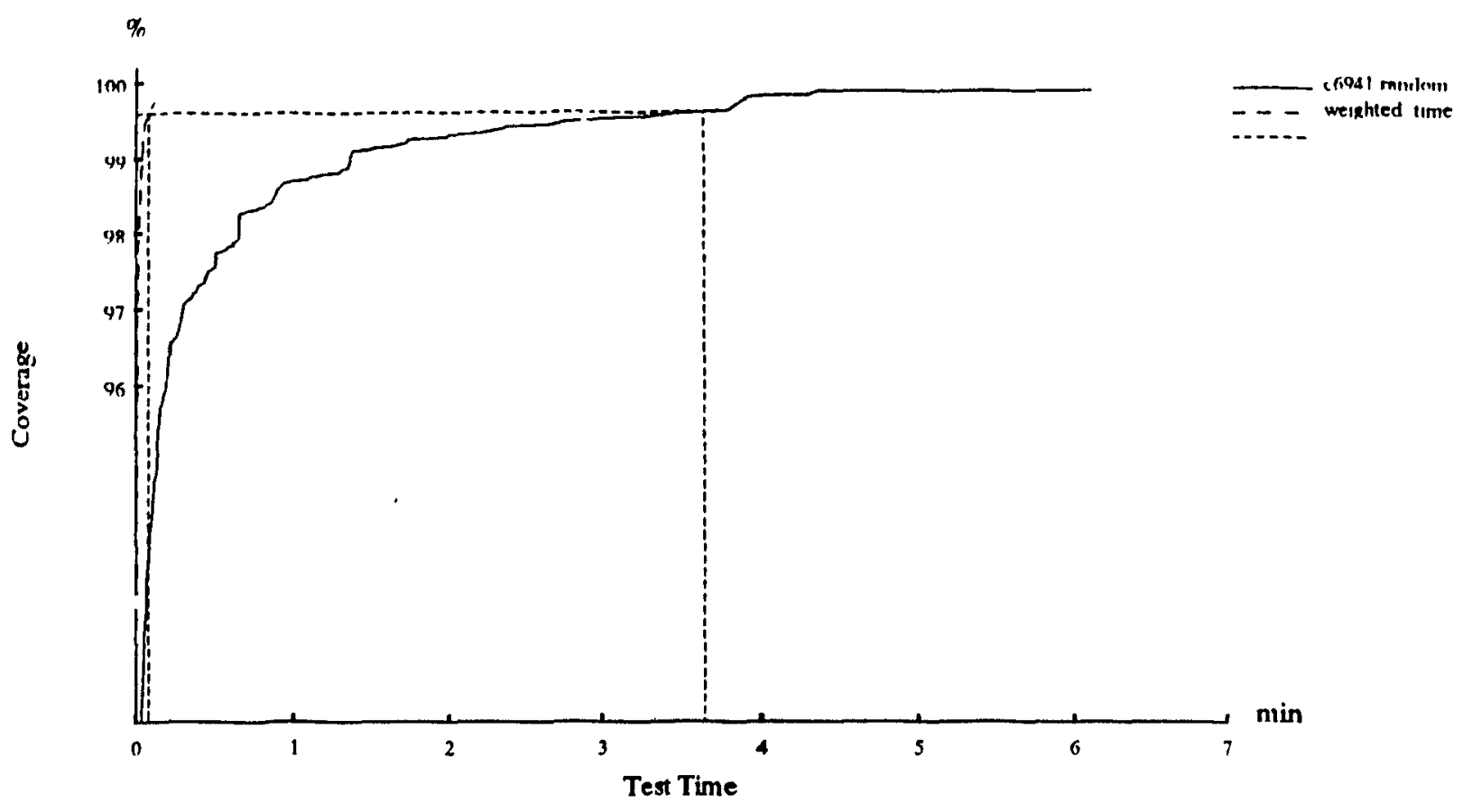

Figure A.1 Coverage vs. Application Time Plots for C6941

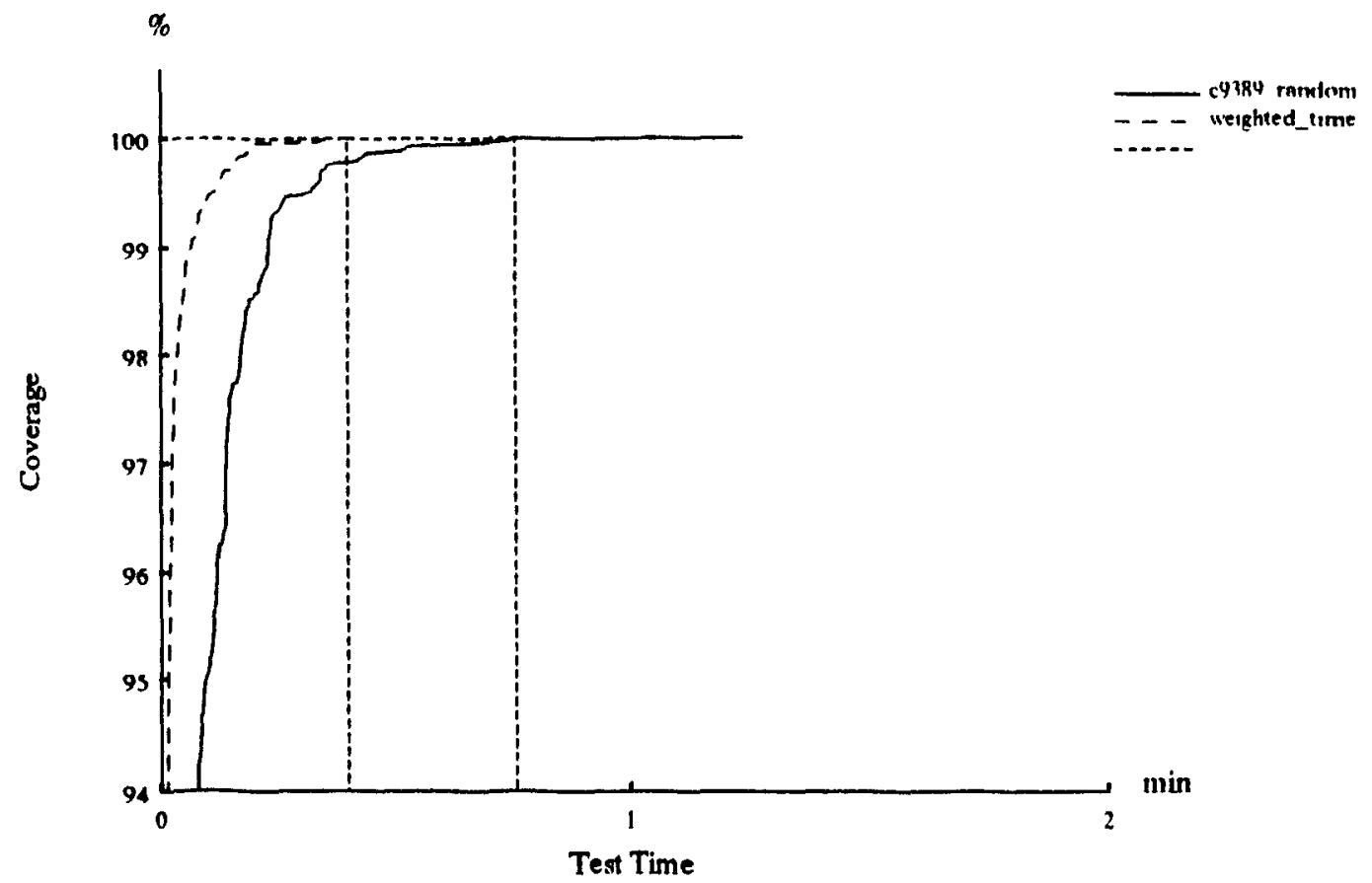

Figure A.2 Coverage vs. Application Time Plots fol C9389 
A Extended Experimental Results

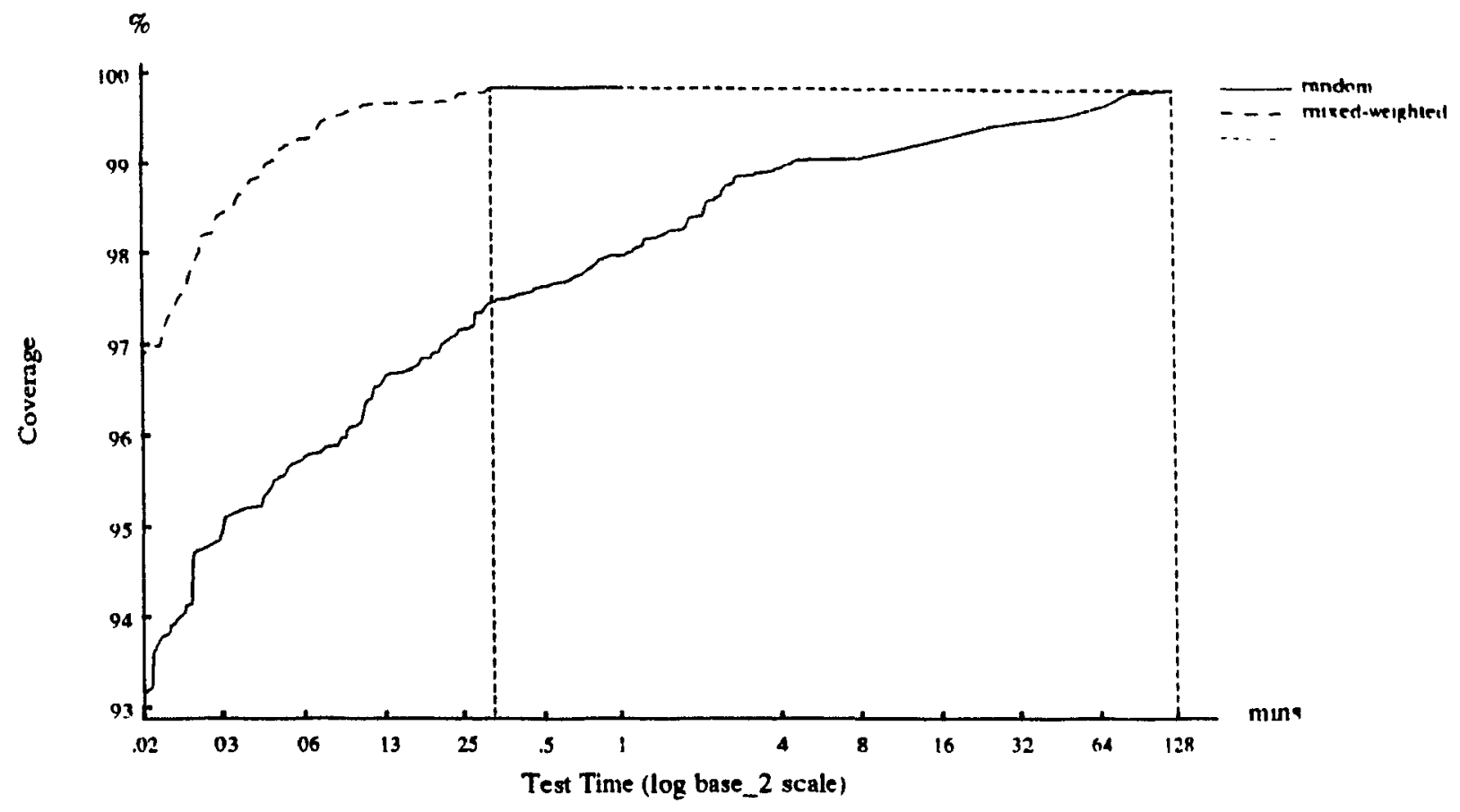

Figure A.3 Coverage vs Application Time Plots for C11657

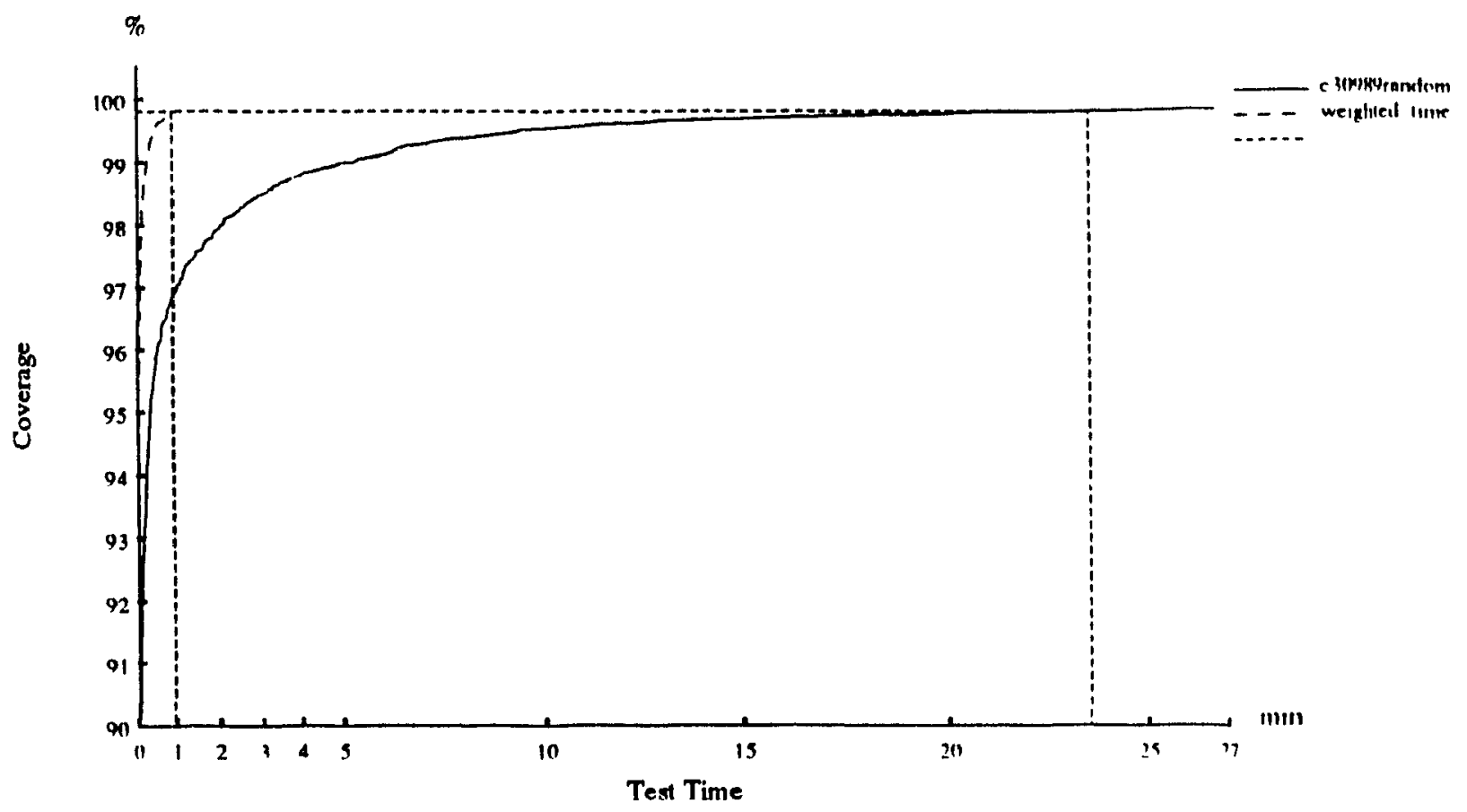

Figure A.4 Coverage vs. Application Time Plots for C30989 
A Extended Experimental Results

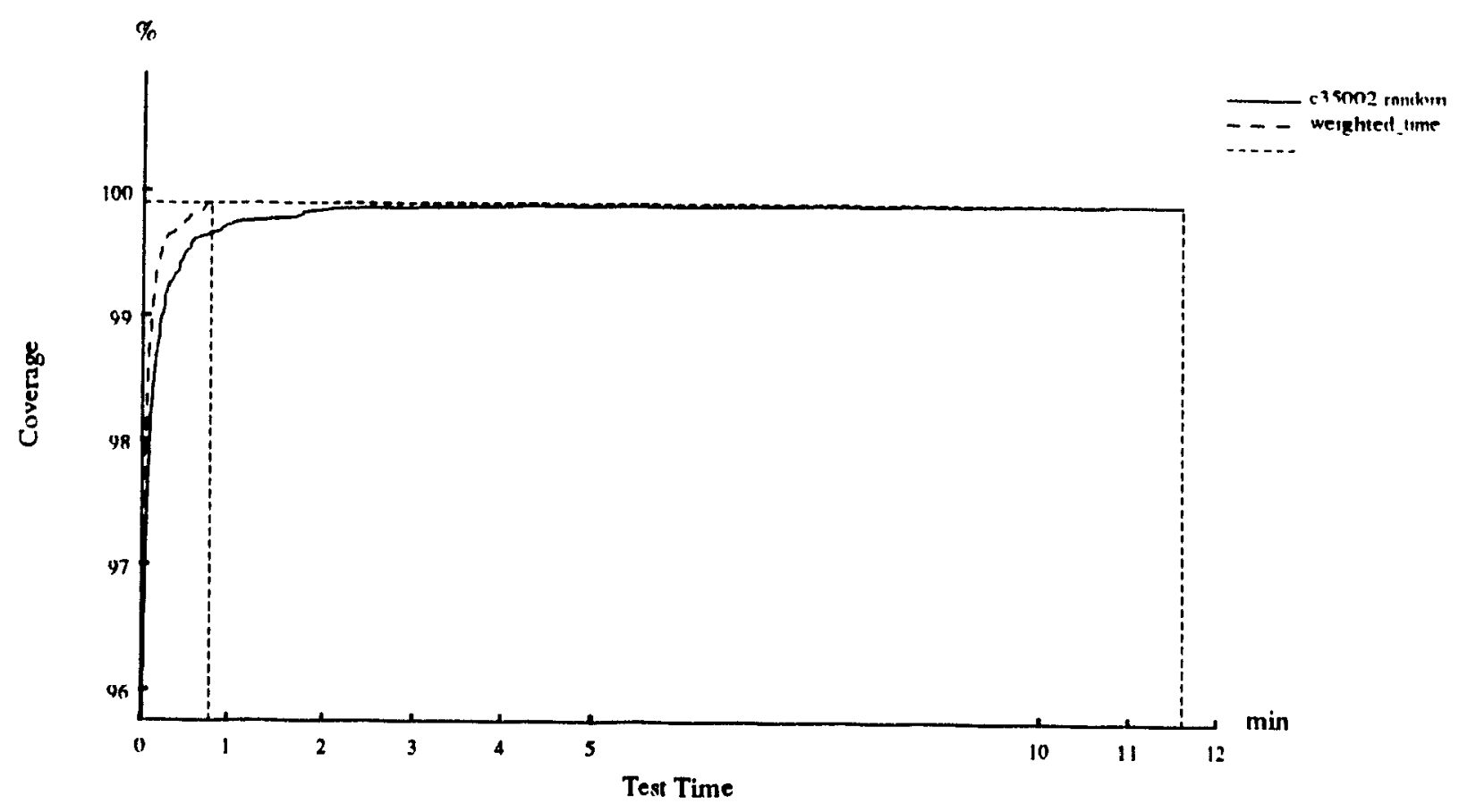

Figure A.5 Coverage vs Application Time Plots for C35002 


\section{References}

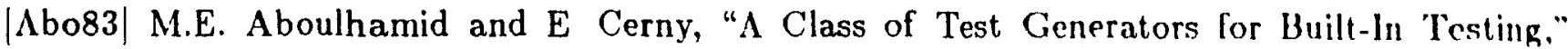
IEEE Trans. Computers Vol. C-32, No. 10, pp 957-959, Oct 198:3

[Aga81] V.K. Agarwal and E. Cerny, "Store and Generate Built-In Self-'lesting Approach," Proc FTCS-11 pp $35-40,1981$

|Agr82| V.D. Agrawal and M.R. Mercer, "Testability Measures - What Do They Tell Ils?." Proc 1982 IEEE Test Conf, pp. 391-396, 1982

|Ait90| R. Aitken, PhD Thes/s, McGill University - in preparation

[Ake76] S. Akers, "A Logic System for Fault Test Generation" IEEE Trans on Computers, wol (-25, NO 6, pp 620-630, June 1976.

And80] H. Ando, "Testing VLSI with Random Access Scan," Proc COMPCON Spring 1980 pp. $50-52,1980$.

|Bar87| P.II. Bardell, W.II. McAnney, J. Savir, Built-In Self-Test for VLSI. Wiley-Interscience. New York, 1987.

|Bas89| R.W Bassett et al., "Low Cost Testing of High Density Logic Components," Proc. ITC 89 , pp $550-557,1989$.

|Bha89| D. Bhattacharya, B.T. Murry, J.P. Hayes, "Iligh-Level Test Generation for VLSI," IEEE Computer. Vol 22, No 4, pp. 16-24, April. 1989

[Boz80] S. Bozorgui-Nesbat and E. J. McCluskey, "Structured Design for Testability to Fliminate 'Tet Pattern Generation," Proc FTCS-10. pp. 158-163, 1980

[Bra84] D. Brahme and J.A. Abraham, "Functional Testing of Microprocessors", IEEE Trans Comp, Vol C-33, pp 475-485, June 1984.

[Brg84] F. Brglez, "On Testability Of Cominational Networks," Proc. IEEE Symp Circiuts Sys . pp 221-225, May 1984.

|Brg89| F. Brglez, C. Gloster, G. Kedem, "Hardware-Based Weighted Random Paltern Ciencration for Boundary Scan," Proc ITC-89, pp 264-274, 1989

|Car82| W.C. Carter, "The Ubiquitous Parity Bit," Proc. FTCS-12, pp 289-296. 1982.

[Che89] K T. Cheng and V.D Agrawal, "An Economical tiran Dosign for Limpurntial I,ogir 'T'met Generation," Proc FTCS-19, pp 28-35, 1989

[Chi87] C.K Chin and E.J McCluskey "Test Length for Pseudorandom Trsting," IEEE Trans Computers, Vol. C-20, pp 1286-1294, Nov. 1971.

[Cox90] H. Cox and J. Rajsk, "A Method to Calculate Necessary Assignments in Algorıthmı" 
Test Pattern Generation" - to appear, Proc ITC-90.

|Dav80| R. David, "Testing by feedback Shift Register," IEEE Trans. Conputers. Vol. C-29. pp. 6fi;8-67:3, July 1980

|Eic87| F.B. Eichelberger et al., "Weighted Random Pattern Testing $\Lambda$ pparatus and Method," Imited States Patent \# 4687988, Aug 18, 1987

$\mid$ Fed86| X. Fedi and R. David, "Some Experimental Results From Random Testing of Microprocessors," IEEE Trans Inst \& Meas. Vol IM-3.5. No 1, pp TR.86 March 1986

|Fro7i| R.A. Frowerk. "Signature Analysis: A New Digital Field Service Method," Hewlett Packard Journal, pp 2-8, May 1977

|Fun89| S.Funatsu. M Kawai, A Yamada, "Scan Design at NEC,", IEEE Design and Test. Vil 6 No. 3. pp 50-57. June 1989.

[Fuj83| H. Fujiwara and T. Shimono, "On the Acceleration of Test Generation Algorithms," IEEE Trans Computers, Vol. C-32, pp1137-1144, Dec. 1983.

|Cilo88| C.S Cilostet and F Brglez, "Boundary Scan with Cellular-Based Built-In Selt-Test," Proc ITC-88. pp 138-145, Washington DC, Sept 1988.

|Gra89| D Graham, Introduction to Multi-Strategy Testing. Genliad Inc.. Concord MA, 1989.

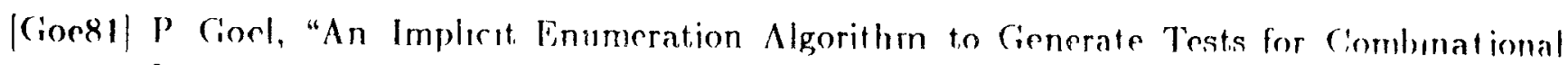
Logic Circuits," IEEE Trans Computers, Vol. ('-30. pp 215-222. Mar 1981

[Col79] I, H Coldstein, "Controllability/Obegervabulity Analysis of Digital Circuits," IEEE Trans Circuits and Systems, Vol. CAS-26, pp. 685-693, Sept 1979

|Gol67| S.W (iolomb. Shift Register Sqeuences, Holden-Day Inc., San Francisco C.1, 1967

[Har87| D. Harel and B. Krishnamurthy, "Is There Hope for Linear Time Fault Simulation?," Proc FTCS-17, pp 28-33, July 1987

|Has88| A Ilassan, J Raskkı and VK Agarwal, "Testıng and Diagnosis of Interconnects using Boundary Scar Architecture," Proc. ITC-88, pp. 126-137. Washongton D C. Sept. 1988.

|IIor89| P D Hortensius, R D. McLeod, "Cellular Automata-Based Signature Analysis for Buill. In Self-Test" Third Techmcal Workshop - New Directions for IC Testing. pp. 117-128. lialifax, Oct. 1988

|Hor89| P.D Hortensius. R.D. McLeod, W Pries. D.M Miller, and H.C. Card, "Cinllular AutomataHased Pseudorandom Number cienerators for Bult-In Sielf-Test." IEEE Trans Comp Aided Design, Vol. 8. pp. 842-859. Aug. 1989

|Hon81| S-J Hong and D L Ostapko "A Simple Procedure to Cenerate Optimum Test Patterna for Parity Logic Networks" IEEE Trans Computers, Vol. C-30, pp. 350-358.

|How89| M.C, Howells, R.C Aitken, and V.K. Agarwal, "Defect Tolerant Interconnert.s for 
VLSI," in Defect and Fault Tolerance in VLSI Systems, I. Koren. ed.. pp. (i5-if. I'lemum Press, New Yolk, 1989.

|Hur88| S.L. Hurst, "A Hardware Consideration of CALBO Testing." Third Techmcal Workshop - New Directions for IC Testing, pp 129-146. Halifax Oc 10k8

|ISC85| ISC AS85 Benchmarks, Sperial Session. "Recent Algorithms for Ciate-level ATP(; wilh fault. Simulation and their Performance Assessment." Proc IEEE Int Symp Corcula Syst . June 1985.

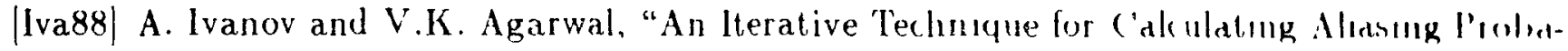
bility of Linear Feedback Shift Registers," Proc FTCS-18, Tokro, Japan, June 1989

|lye89| VS Iyengar and D Brand, "Synthesis of Pseudo-Random Pallern Testahle Destgun." Proc. ITC-89, pp. pp. 501-508, Washington DC:, Aug. 1989.

|Kar85| M Karpovsky, ed. Spečral Technıquesand fault Detectıon. Academic I'ress, Lomdon

|Kim88| K. Kim, D.S. Ha, J.G. Tront, "On Using Signature Registers as Pscudorandom Paflern Generators in Built-in Self-Test," IEEE Trans Comp Alded Design Vol T.pp (3/4-1)2*. Aug 1988 .

|Koe79| R Koenemann. J Mucha and G Zwiehoff, "Built-in Logic Mlock Obaervation Terhniques." Proc ITC-79, pp. 37-41, Chen'v Hill NJ.. 1979

[Kra87] A. Krasniewskiand S Pilarski, "Circular Self-Test Path. A low-C'ost. BIST Techiniclue." Proc 24th Des gn Automation Conf., pp 407-415. 1987

|Kri85| B. Krishnamurthy and C. Sheng, "A New Approach to the Use of Testability Analvsis in Test Generation, Proc ITC-85, pp. 769-778, 1985

|Kri84| B. Krishnamurthy and S.B. Akers, "On the Complexity of Estimating the Size of a Trsi Set," IEEE Trans Computers, Vol. C-33, No. 8, 1984

|Kub83| J.R. Kuban and W.C. Bruce, "The MC6804P2 Built-In Self-Test," Proc ITC.83, 1) $295-300,1983$

[Kaw89] M. Kawamura, K. Mera, A. Tateishi, "A Study on Efficient Test Cieneralion for laarg" Sican-based Circuits," 12th IEEE Workshop on Design for 'Testability. Vail, ('). A mil 198:.

|Lis87| R. Lisanke, F. Brglez, A. Degeus and D. Gregory. "Testal,ility- D)ıven Rinrlonn Tint Pattern Generation." IEEE Trans. CAD, Vol. CAD-6. No.6. Nor 1987.

|Maa88| F Maamarı and J Rajskı, "A Fault Simulation Based on sitem Regırms." Pror ICCAD. pp. 170-173, Nov 1988.

|Man89| W.R. Mann, "R96MFX 'Test Strategy," Proc ITC-89, pp 611-614, Washıngt,on I) ' . Aug. 1989.

[McC84] E.J McCluskey, "Verification Testing - A Pseodo-Exhaustıve Test Terhnique," IEEE 
Trans Computers, Vol C-33, pp. 541-546, June 1984.

|McC85a| E.J. McCiuskey, "Bulit-In Self-Test Techniques," IEEE Design and Test. Vol. 2. No. 2. pp 21-28, April 1985

|Mr C85) b E.J. MrCluskey, "Bulit-In Self-Test Structures," IEEE Design and Test, Vol. 2, No. 2. pp 29-36. April 1985

|Mei71| K ' I Me1, "Bridgng and Stuck-at Faults," IEEE Trans Computers, Vol. C'-23. pp). $720-727$. J11 1974

|Nad88| B Nadcau-Dostıe, A. Ermarkaryan, L McNaughton, "Practical Scan Lesign for ASIC'a," Third Technical Workshop - New Directions for IC Testıng, pp 169-184. Halifax. Ort 1988

|Nil80| N.J Nilsson, "Principles of Artificial Intelligence," Morgan Kaufman Pub. Calif., 1980.

|Par89| K P' Parker. "The Impact of Boundary Scan on Board 'Test," IEEE Design and Test, p) 18-30. Aug 1989.

|Pet72| IW W Peterson and E.J. Weldon, Error Correcting Codes. 2nd ed . MIT Piess, Cambridge $M \Lambda, 1972$

|P'ra88| M M l'radhan, E.J O'Brien, S.L. Lam and J Beausang, "Circulaı BIST with Partial Sical" Proc ITC-88, pp 719-729, Washington DC, Sept. 1988.

|Rot66| J P Roth, "Diagnosis of Automata Fallures: A Calculus Method," IBM J Res Devel. vol 10, pp. 278-281, July 1966.

|Siav84| J Savir, (is Ditlow and P Bardell, "Random Pattern Testability," IEEE Trans Computers. Vol (-33, pp. 79-90, Jan. 1984.

|Sich75| II D Sirhmurmann, E Lmdlhoom, R.G Carpenter, "The Weighterl Random Test Pattern Generalor," IEEE Trans Computers. Vol. C-24, pp. 695-700, July 1975.

|Srh88| M II Schulz and F Auth, "Advanced Automatic Test Pattern Cieneration and Rerlundancy Identification," Proc FTCS-18. Tokyo, Japan, June 1988.

|Sel68| F F. Seliers, M.Y Hstao and L.W Bearnson, "Analysing Errors with Boolean Difference," IEEE Trans. Computers, Vol. C-17, pp. 676-683, luly 1968

|Sicr88| M. Serra, D. Miller. J C. Muzio, "Linear Cellular Automata and LFSiRs Are Isomorphic," Third Technical Workshop - New Directions for IC Testing, pp 213-223, Halifax, Oct 1988

$\mid$ Set85| S (' Seth and VD Agrawal, "Cutting Chip-Testing Costs," IEEE Spectrum. pp. 38-45, Apr. 1985.

|She85| Shen J P, Maly W, Ferguson J F, "Inductıve Fault. Analysis of MOS Integralied r,iicuits," IEEE Design and Test. pp. 13-26, December 1985. 
[Sia88] F. Siavoshi, "WTGPA: A Novel Weighted Test-Pattern Gencration appronch for $\backslash$ I, I Built-I Self-Test", Proc ITC-88, pp. 256-262, Washungton D)'. Simpt I GXs

[Smi79] J.E. Sr.lith, "Detection of Faults in Programmable Logic Arrays", IEEE Trans Comp . Vol. C: 28 , pp 845-852, Nov. 1979

[Ste77] J H Stewart, "Fulure Testing Of Large LSI Circuit Cards," Proc 1977 Semicondurtor Test Symp.pp 6-15, Oct 1977

|Tot88| K. Totton and S. Shaw. "Self-Test. The Solution to the VLSI Test l'roblem"." IEE Proceedings, Vol. 135. Pt. E. No. 4, pp. 190-195, July 1988.

|Ude88| J.G. Udell, "Reconfigurable IJardware for Pseudo-Exhaustive 'Test," Proc ITC.88. W" 522-530, Washington DC, Sept. 1988.

|Wad78| R L. Wadsack. "Fault Modelling and Logic Simulation of CMOS and MOS luteglater] Circuits," Bell Sys Tech Jour. Vol 57, pp 1449-1474,1078

|Wai85| J.A. Waicukausk, E.B Eichelberger, D O Forlenza, E lindbloom and T Mc('arthr, " $\Lambda$ Statistical Calculation of fault Detection Prohabilities hy Fast Fault Simulation." Proc ITC-85. Philadelphia, P A, 1985

[Wai88| J.A. Waicukauski, V.P. Gupta and S T Patel, "Fault detection Fffectireness of Weighterl

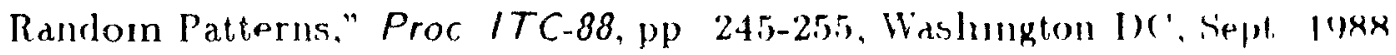

|Wan86| L.T. Wang and E.J McCluskey, "A Ilvbrid Design of Maximum-Lenglh Sequence (innerators," Proc ITC-86, pp 25-?7. Sept 1986

|Wil83| T.W. Williams and K.P. Parker, "Design for Testability - A Survev," Proc. IEEE. Vol. 71, pp 98-112, Jan 1983.

|Wil86| T.W. Williams, W. Daehn, M. Gruetzner, C.W. Starke, "Comparison of Aliasing Lrrors for Primative and Non-Primative Polynimials," Proc ITC 1986, Washington I) (: , Iuxfi

|Wol83| S. Wolfram, "Statistical Mechanics of Cellular Automala". Rev of Modern Phys, Vol 5.5. pp 601-644, 1983.

[Wun87] HI -J. Wunderlich, "Self-Test using Unequiprohable Random Pallans," Proc Fault Tolerant Computing Symposium. pp. 258-263. July 1987

[Wun88] II.-J Wunderlich, "Multiple Distributions for Based Random Test Pallerms," Pror ITC.88, pp. 236-244, Washington DC. Sept. 1988. 